\title{
Liposuction Assisted Abdominoplasty (LAA)
}

Citation for published version (APA):

Brauman, D. (2019). Liposuction Assisted Abdominoplasty (LAA): A Comprehensive Approach to Body Contouring. [Doctoral Thesis, Maastricht University]. ProefschriftMaken Maastricht. https://doi.org/10.26481/dis.20190625db

Document status and date:

Published: 01/01/2019

DOI:

$10.26481 /$ dis.20190625db

Document Version:

Publisher's PDF, also known as Version of record

\section{Please check the document version of this publication:}

- A submitted manuscript is the version of the article upon submission and before peer-review. There can be important differences between the submitted version and the official published version of record.

People interested in the research are advised to contact the author for the final version of the publication, or visit the DOI to the publisher's website.

- The final author version and the galley proof are versions of the publication after peer review.

- The final published version features the final layout of the paper including the volume, issue and page numbers.

Link to publication

\footnotetext{
General rights rights.

- You may freely distribute the URL identifying the publication in the public portal. please follow below link for the End User Agreement:

www.umlib.nl/taverne-license

Take down policy

If you believe that this document breaches copyright please contact us at:

repository@maastrichtuniversity.nl

providing details and we will investigate your claim.
}

Copyright and moral rights for the publications made accessible in the public portal are retained by the authors and/or other copyright owners and it is a condition of accessing publications that users recognise and abide by the legal requirements associated with these

- Users may download and print one copy of any publication from the public portal for the purpose of private study or research.

- You may not further distribute the material or use it for any profit-making activity or commercial gain

If the publication is distributed under the terms of Article $25 \mathrm{fa}$ of the Dutch Copyright Act, indicated by the "Taverne" license above, 


\section{Ph.D. Thesis}

Liposuction Assisted Abdominoplasty (LAA)

A Comprehensive Approach to Body Contouring

Daniel Brauman, MBBS, MD, FACS 
(C) copyright Daniel Brauman, Maastricht 2019

Printing: ProefschriftMaken || www.proefschriftmaken.nl

ISBN 978-94-6380-377-9

All rights reserved. No part of this publication may be reproduced, stored in a retrieval system or transmitted, in any form or by any means, electronic, mechanical, photocopying, recording or otherwise, without prior permission of the author or the copyright-owning journals for previous published chapters. 


\section{Maastricht University}

\section{Liposuction Assisted Abdominoplasty (LAA) A Comprehensive Approach to Body Contouring}

\section{DISSERTATION}

To obtain the degree of Doctor at Maastricht University on the authority of the Rector Magnificus

Prof.dr. Rianne M. Letschert

in accordance with the decision of the board of deans, to be defended in public on Tuesday $25^{\text {th }}$ of June 2019 at 12.00 hours

by

Daniel Brauman, MBBS, MD, FACS 


\section{Supervisors:}

Prof. dr. B. van der Lei, UMCG

Prof. dr. R.R.W.J. van der Hulst

\section{Assessment Committee:}

Prof. dr. M.E.A. Spaanderman, chair

Prof. dr. G.L. Beets

Dr. J. Stevens, Velthuis Kliniek

Dr. V. Tanaydin, Betan Clinics 
The endeavor to understand is the first and only basis of virtue.

\section{Spinoza}





\section{Contents}

Chapter 1

Introduction Overview and Outline of this Thesis

\section{Chapter 2}

21

Lipoplasty: A Case for a Low-volume Procedure

Brauman D. Aesth. Surg. J. 2000; 20 (5): 373-379

Chapter 3

Liposuction Abdominoplasty: An Evolving Concept

Brauman D. Plast. Reconstr. Surg. 2003; 112:288-298

\section{Chapter 4}

Diastasis Recti: Clinical Anatomy

Brauman D. Plast. Reconstr. Surg. 2008; 122: 1564-1569

\section{Chapter 4a Letters}

Diastasis Recti: Clinical Anatomy

Plast. Reconstr. Surg. June 2009; 123: $6: 1885$

Factors that May Influence Failure of the Correction of the Musculoaponeurotic

Deformities of the Abdomen

Plast. Reconstr. Surg. July 2009; 124:1: 334-335

\section{Chapter 5}

Liposuction Abdominoplasty: An Advanced Body Contouring Technique

Brauman D. Plast. Reconstr. Surg. 2009; 124:1685-1695

\section{Chapter 6}

A Case for Local Anesthesia and Prevention Protocols for Curbing DVT in Abdominoplasty Brauman $D$, van der Hulst $R$, van der Lei $B$

\section{Chapter 7}

Liposuction Assisted Abdominoplasty (LAA): An Enhanced Abdominoplasty Technique Brauman $D$, van der Hulst $R$, van der Lei $B$.

Plast. Reconstr. Surg. Global open

September 2018, Volume 6, Issue 9- p 1940

\section{Chapter 8}

Abdominoplasty with Circumferential Liposuction, DVT, Local Anesthesia: How Much Proof is Needed?

Brauman D, van der Hulst R, van der Lei B.

Plast. Reconstr. Surg. In Press

\section{Chapter 9}

General Discussion and Future Perspectives

\section{Chapter 10}

Valorization Addendum 

Chapter 1.

Introduction Overview and Outline of this Thesis

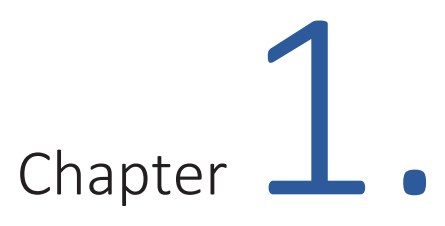

\author{
Introduction \\ and \\ Outline of this Thesis
}





\section{Chapter 1.}

\section{Introduction and Outline of this Thesis}

\section{Background}

The term body contouring refers to plastic surgical procedures that modify the shape of the human body as in abdominoplasties, incisional body surgeries and liposuction.

Abdominoplasties were originally devised during the late 19th and early 20th centuries in Europe and the US to repair large umbilical hernias and remove disabling abdominal adiposities. Demars and Marx (1890), Gaudet and Morestein (1905) and Dessjardin (1911) in France, Kelly in the US (1899), and Weinhold (1909), Jolly (1911) and Shepelmann (1918) in Germany, described their techniques for resections of large "apron-like" abdominal skin and fat collections. ${ }^{1-10}$ The resections described were vertical transverse and combined vertical and transverse resections. The surgery relieved discomfort, skin rashes and infections - Intertrigo and aided in weight control. Babcock (1916), appears to have been the first to correct the abdominal muscular-fascial laxity using a buried silver chain. ${ }^{11}$ Thereafter, plications of the deep fascia became integral to abdominoplasties. Approximating the recti muscles in the midline -diastasis repair, resulted in firmer flatter abdomens and increased core strength, rendering the abdominal muscles more efficient, thereby supporting the back.

\section{Safety Issues in Body Contouring}

Early abdominoplasties were referred to as lipectomies or dermo-lipectomies and for the most part did not utilize significant undermining. As modern abdominoplasty techniques evolved, the preferred incisions became low transverse incisions of various shapes to hide the incisions and to prevent scar contractures. ${ }^{12-14}$ As a result of improved surgical and anesthetic techniques, abdominoplasties became mainstream surgeries to be used as aesthetic procedures and attention became focused on their safety and efficacy. ${ }^{15-19}$ In 1973 Grazer reported a 7.14\% incidence of venous thromboembolism (VTE) when abdominoplasty was combined with intraabdominal procedures and in 1977 Grazer and Goldwyn reported an overall complication rate of $14.6 \%$ and a VTE rate of $1 \% 1 .^{15,16}$ Van Uchelen et al. reported a wound complication rate of 
$15.3 \%$ in their female patients and no deep vein thrombosis (DVT) ${ }^{17}$ and Stewart et al. reported a reoperation rate of $24 \%$ and a $0.36 \%$ rate of VTE. ${ }^{18}$ Hatef et al., reviewing abdominoplasties combined with liposuction and other procedures, concluded that the VTE incidence of abdominoplasty alone was $\mathbf{0 . 3 4 \%}$, abdominoplasty with liposuction or other plastic surgical procedure $0.67 \%$, and abdominoplasty with body lift procedures $3.4 \%{ }^{19}$

Deep Vein Thrombosis (DVT) and Pulmonary Embolism (PE) are often classified together in the literature as - Venous thromboembolism (VTE) and represent a serious and sometimes fatal complication of abdominoplasty. Abdominoplasties are most often performed under general anesthesia, which is a known risk factor for DVT/PE. ${ }^{20,21}$ However, the incidence of DVT/PE in abdominoplasty is higher than in other plastic surgical operations performed under general anesthesia suggesting that the abdominoplasty procedure is an independent risk factor for DVT/PE. ${ }^{22-24}$ DVT's effects are long-term. The deep thrombophlebitis destroys valves in the deep venous system, predisposing an individual to a life-long increase in the risk for stasis and recurring thrombosis. Additionally, a post-phlebitic extremity may develop, causing life-long morbidity. The incidence of other post-abdominoplasty complications such as seroma and flap necrosis as well as the incidence of post-operative sequelae such as scars and scar contractures, is not negligible. Therefore, techniques and approaches were needed, to reduce complications and untoward sequelae while maintaining functional and pleasing results.

The introduction of liposuction by Illouz during the early 1980's revolutionized body contouring. Illouz, not only introduced the technique but also recognized that it could be utilized as an abdominoplasty technique he named - Suction Abdominoplasty. ${ }^{25}$ The aesthetic benefits of combining liposuction and abdominoplasties led to surgeons to combine these procedures in various forms during the 1980's and 1990's, nevertheless the plastic surgery literature was advising caution as liposuction continued to be considered a risk factor to flap circulation. ${ }^{26-31}$

General anesthesia complications are comorbidities of all surgeries, especially during prolonged and complicated procedures performed on individuals who are or who turn out to be high risk because of unknown risk factors. In abdominoplasty under general anesthesia, postoperative respiratory difficulties have resulted from an increase in intra-abdominal pressure and the anesthesia. ${ }^{32}$ Seroma, the most common complication of abdominoplasty averages about $10 \%$ 
in frequency, often necessitating prolonged post-operative suction drainage and in difficult or protracted cases sclerotherapy-ablation and/or re-operation. ${ }^{33-36}$ Dillerud, linked skin necrosis to wide undermining. Skin necrosis is a frequent problem with abdominoplasty and is commonly reported as a - wound complication. ${ }^{26}$ Sensory changes of the abdomen and thigh have been reported by van Uchelen et al. and a degree of numbness of the lower abdomen almost always accompanies abdominoplasty procedures. ${ }^{18}$ Furthermore, Long and sometimes geometrically designed incisions as well as scar contractures continue to plague abdominoplasties. ${ }^{37,38}$

To minimize abdominoplasty complications and reduce the length and extent of scars, limited abdominoplasties with shorter scars and limited undermining have been described by Wilkinson and Swartz, Greminger, Eaves et al., Zukowski et al. and Lockwood. ${ }^{39-44}$

Finally, procedures combining abdominoplasties with breast surgeries, liposuction and other plastic surgical operations are being performed with some frequency. Generally, combing any surgical procedures increases the incidence of problems and complications and in the case of abdominoplasty, performing a hysterectomy in conjunction with an abdominoplasty had been found to be particularly troublesome.

\section{About this Thesis}

Aesthetic surgery aims to construct a pleasing aesthetic result without complications, and although the complete elimination of complications is unlikely, the bar remains high and the expectation for minimal surgical problems remains.

The need for more safety in body contouring became urgent when publicized deaths in the US from anesthetic and liposuction complications during the 1990's resulted in regulatory oversight and a reluctance by many to undergo these procedures. ${ }^{45-49}$ Furthermore, plastic surgeons were indirectly cited in the cause of deaths from fluid overload, lidocaine toxicity, anesthesia complications and DVT, because in contrast to dermatologists, plastic surgeons by and large used general anesthesia for liposuction. Moreover, liposuction began to be used together with abdominoplasty and the early experience with this combination was problematic. ${ }^{26,40,51}$

In order to avoid DVT in body contouring, the author began to perform all liposuction surgeries 
under local anesthesia as of 1990 and all abdominoplasties under local anesthesia since 1996. Additionally, all liposuctions were studied prospectively from 1990-2000 and all abdominoplasties from 1996 to the present. The resultant database provided prospectively gathered evidence for the articles cited in this thesis and offered ongoing feedback as concepts were being developed and corroborated.

\section{Aim of this Thesis}

The approach to body contouring described here, -Liposuction Abdominoplasty, has been recently renamed - Liposuction Assisted Abdominoplasty (LAA), to better distinguish it from other abdominoplasty approaches. This thesis aims to open this approach to scientific scrutiny and debate. It also aspires to broaden the use of this approach, since only through wide-spread use can the benefits of the techniques presented here be verified or refuted.

Liposuction Assisted Abdominoplasty is also a series of concepts. Both the technique and the concepts will be described and discussed in this thesis and it is hoped that the concepts will become evident and verified through the use of the technique.

Deep Vein Thromboses and pulmonary embolisms are dangerous and cause lifelong disabilities. Complete elimination of surgical DVT/PE is a far-reaching but perhaps an attainable goal. This thesis describes strict - DVT prevention protocols that have been shown effective for 28 years in a large number of patients without the use of chemoprophylaxis. It is hoped that these protocols be adopted and tested by others so that their effectiveness may be proven or disproven.

The distinction between - abdominoplasty with liposuction and - liposuction-abdominoplasty (or LAA) is aimed to be clarified since the literature often does not distinguish between these seemingly interchangeable terms. The fundamental concept of the liposuction-abdominoplasty (LAA) technique is that liposuction preserves all but the smallest blood vessels and enhances flap circulation relative to current surgical dissection techniques using cutting or cautery to dissect and separate tissue. Therefore, liposuction-abdominoplasty (LAA), ought to be defined an abdominoplasty technique that utilizes liposuction as a -dissection-tool to dissect free and separate the abdominal flap from the muscular abdominal wall, not merely liposuction with or as an adjunct to abdominoplasty. 
Abdominoplasty under local anesthesia has been practiced by surgeons since about 1996. This approach nearing approximately 22 years has been shown to be safe and effective; however, abdominoplasty is still mostly done under general anesthesia because the approach is efficient, and the anesthesia care is straight forward. Nevertheless, under local anesthesia DVT/PE is rare. This thesis aims at increasing the acceptance of abdominoplasty under local anesthesia by advocating for it and adding a significant number of consecutive cases performed without anesthetic complications, DVT/PE, respiratory complications or hospital transfers.

\section{Outline of this Thesis}

This thesis was written in chronological order. Chapters were arranged by dates of publication with each chapter describing a progression in - technique in conjunction with important theoretical concepts.

Chapter 1. This chapter briefly reviews the history of abdominoplasty and discusses in some detail its safety issues. It then briefly introduces the LAA approach to body contouring as a means for a safer and a more predictable approach.

Chapter 2. A 2000 Aesthetic Surgery Journal article was written in response to reports of deaths and complications during the 1990's from general anesthesia complications, DVT/PE, fluid overload and lidocaine toxicity, that resulted from wet liposuction techniques. The article proposed solutions to these issues. ${ }^{52}$

Chapter 3. A 2003 article introduced the Liposuction-Abdominoplasty (presently LAA) technique as an alternative to traditional abdominoplasty. The procedure, performed under local anesthesia with sedation to minimize DVT, introduced the concept of a well perfused, sliding, mobile, sensate abdominal flap, separated from the underlying muscular abdominal wall by liposuction with minimal undermining. The article also described the assessment of the intraabdominal fat content and its impact on surgical decision making and outcome. ${ }^{53,54}$

Chapter 4. 2008's Diastasis Recti: Clinical Anatomy, studied abdominal wall laxity and protrusion and its relation to diastasis recti. It challenged the notion that the presence and size of diastasis recti is a reliable indicator of abdominal wall protrusions and argued that an enduring diastasis 
repair should not be universally accepted as a yardstick by which the success of an abdominoplasty is measured..$^{55}$

Chapters 4a. This chapter features correspondence and exchange of opinions on diastasis repairs. ${ }^{56,57}$

Chapters 5. In 2009, a PRS article described in detail the Liposuction-Abdominoplasty (LAA) technique, introducing concepts of hydro-lipo-dissection, supporting tissue boost to flap perfusion, tapered segmental diastasis repairs, local anesthesia for DVT prevention, sedation principles, a definition for seroma, and disruption of the skin retaining ligaments. ${ }^{58}$

Chapters 6. 2018, a treatise in article layout suggesting that local anesthesia with sedation is thus far the most effective DVT prevention protocol, reviewing relevant literature, and describing a strict DVT protocol that has been found effective when utilized in its entirety. ${ }^{59}$

Chapter 7. A PRS Global Open (2018) article summarized the experience with LAA in 593 consecutive patients, describing with video the operative technique and concluding that LAA is a safe and effective body contouring technique. LAA, has routinely incorporated circumferential torso and other areas liposuction and has commonly included buttock fat grafting and hernia repair. The extensive clinical experience with LAA suggests longer flap excursions and improved perfusion as well as the realization of goals for improved safety. ${ }^{60}$

Chapter 8. 2019, a PRS letter to the editor suggesting that abdominoplasty under local anesthesia and sedation has already passed the burden of proof and should be recognized effective in preventing DVT. ${ }^{61}$

Chapter 9. General discussion and future perspectives

Chapter 10. Valorization Addendum. Acknowledgement. About the author

\section{References}

1. Demars MM, Marx M. Surgical treatment of obesity. Prog Med. 1890; 11:283

2. Gaudet F, Morestin H. French congress of surgeons. Paris: Hoffmann; 1905. 
3. Morestin, Amédée. "La restauration de la paroi abdominale par résection étendue des téguments et de la graisse sous-cutanée et le plissement des aponévroses superficielles envisagée comme complément de la cure radicale des hernies ombilicales." PhD diss., Société Générale d'Imprimerie et d'Édition Levé, 1912.

4. Resection de la couche adi d'obesite extreme (lipectomie). P Desjardins - Rapport par Dartigues. Paris Chirurg, 1911.

5. Kelly H. Report of gynaecological cases. Bull J Hopkins Hosp. 1899;10:197.

6. Excision of the fat of the abdominal wall lipectomy. HA Kelly - Surg Gynecol Obstet, 1910

7. Bauchdeckenplastik. S Weinhold - Zentralbl F Gynak, 1909.

8. Jolly, R. "Abdominoplasty." Berl Klin Wochenschr 48 (1911): 1317.

9. Über Bauchdeckenplastik mit besonderer Berücksichtigung des Hängebauches. E Schepelmann Beitr Klin Chir, 1918.

10. Callia WEP: Contribuição para o estudo da correção cirúrgica do abdome pêndulo e globoso-técnica original. Tese de Doutoramento apresentada à Faculdade de Medicina da USP, São Paulo, 1965.

11. Babcock, W Wayne. The correction of the obese and relaxed abdominal wall with especial reference to the use of buried silver chain: With eleven illustrations Tensile strength of good raw and sterilized Catgut, slow pull. The American Journal of Obstetrics and Diseases of Women and Children (18691919); New York74.4 (Oct 1, 1916): 596.

12. Pitanguy I: Abdominoplastias. O Hospital71:1541, 1967.

13. Pitanguy, I., et al. "Aspectos atuais em lipectomia abdominal." Rev Bras Cir 64.5/6 (1974): $147-67$.

14. Regnault P: Abdominal dermolipectomies. Clin Plast Surg2:411, 1975.

15. Grazer FM. Abdominoplasty. Plast Reconstr Surg. 1973;51:617-623.

16. Grazer FM, Goldwyn RM. Abdominoplasty assessed by survey with emphasis on complications. Plast Reconstr Surg. 1977;59:513-517.

17. Stewart KJ, Stewart DA, Coghlan B, Harrison DH, Jones BM, Waterhouse N. Complications of 278 consecutive abdominoplasties. J Plast Reconstr Aesthet Surg. 2006;59(11):1152-5.

18. van Uchelen, J.H. Werker, P.M.N., Kon, M. Complications of Abdominoplasty in 86 Patients. Plast. Refconstr. Surg. 107: 1869, 2001.

19. Hatef DA, Trussler AP, Kenkel JM. Procedural risk for venous thromboembolism in abdominal contouring surgery: A systematic review of the literature. Plast Reconstr Surg. 2010;125:352-362.

20. Reinisch, J. F., Bresnick, S. D., Walker, J. W. T., and Rosso, R. F. Deep venous thrombosis and pulmonary embolus after face lift: A study of incidence and prophylaxis. Plast. Reconstr. Surg. 107: 1570, 2001.

21. Stuzin, J. M., Baker, T. J., and Baker, T. M. Deep venous thrombosis and pulmonary embolus after face lift: A study of incidence and prophylaxis (Discussion). Plast. Reconstr. Surg. 107: 1576, 2001 
22. Daane, S. P., and Rockwell, W. B. Analysis of methods for reporting severe and mortal lipoplasty complications. Aesthetic Plast. Surg. 23: 303, 1999.

23. Venturi, M.L., Davison, S.P., Caprini, J.A. Prevention of Venous Thromboembolism in the Plastic Surgery Patient: Current Guidelines and Recommendations Aesthet Surg J (2009) 29 (5): 421-428.

24. Keyes, G.R. Commentary on: Venous Thromboembolism in the Cosmetic Patient: Analysis of $129,007$. Aesthetic Surgery Journal. 2017, Vol 37(3) 350-352.

25. Illouz Y-G. A new safe and aesthetic approach to suction abdominoplasty. Aesthetic Plast Surg. 1992;16:237-245.

26. Dillerud, E. Abdominoplasty combined with suction lipoplasty: A study of complications, revisions, and risk factors in 487 cases. Ann. Plast. Surg. 1990; 25: 333.

27. Matarasso, A. Liposuction as an adjunct to a full abdominoplasty. Plast. Reconstr. Surg. 1992; 95 : 829.

28. Matarasso, A. Liposuction as an adjunct to a full abdominoplasty revisited. Plast. Reconstr. Surg. 200; 106: 1197.

29. Baroudi, R. Liposuction as an adjunct to a full abdominoplasty revisited (Discussion). Plast. Reconstr. Surg. 2000; 106: 1203.

30. Shestak, K. C. Liposuction as an adjunct to a full abdominoplasty revisited (Discussion). Plast. Reconstr. Surg. 2000; 106: 1205.

31. Heppe, H. P. Combined liposuction with abdominoplasty (Letter). Plast. Reconstr. Surg. 2001; 108: 577.

32. Blatnik JA. Predicting Severe Postoperative Respiratory Complications following Abdominal Wall Reconstruction. Plast Reconstr Surg . 2012; 130: 836.

33. Baroudi R, Ferreira CAA. Seroma: How to avoid it and how to treat it. Aesthet Surg J. 1998; 18:439.

34. Chaouat M, Levan P, Lalanne B, Buisson T, Nicolau P, Mimoun M. Abdominal dermolipectomies: Early postoperative complications and long-term unfavorable results. Plast Reconstr Surg. 2000; 106:1614.

35. Nahas FX, Ghelfond C, Ferreira LM. Does quilting suture prevent seroma in abdominoplasty? Plast Reconstr Surg. 2007; 199:1060.

36. Grazer FM, Goldwyn RM. Abdominoplasty assessed by survey with emphasis on complications. Plast Reconstr Surg. 1977; 59:513.

37. Regnault, P. Abdominoplasty by the $W$ technique. Plast. Reconstr. Surg. 55: 265, 1975.

38. Regnault, P. Abdominal dermolipectomies. Clin. Plast. Surg. 2: 411, 1975.

39. Wilkinson, T. S., and Swartz, B. E. Individual modifications in body contour surgery: The limited abdominoplasty. Plast. Reconstr. Surg. 77: 779, 1986. 
40. Wilkinson, T. S. Limited abdominoplasty techniques applied to complete abdominal repair. Aesthetic Plast. Surg. 18: 49, 1994.

41. Greminger, R. F. The mini-abdominoplasty. Plast. Reconstr. Surg. 79: 356, 1987.

42. Eaves, F. F., III, Nahai, F., and Bostwick, J., III. Endoscopic abdominoplasty and endoscopically assisted miniabdominoplasty. Clin. Plast. Surg. 23: 599, 1996.

43. Zukowski, M. L., Ash, K., Spencer, D., Malanoski, M., and Moore, G. Endoscopic intracorporal abdominoplasty: A review of 85 cases. Plast. Reconstr. Surg. 102: 516, 1998.

44. Lockwood, T. High-lateral-tension abdominoplasty with superficial fascial system suspension. Plast. Reconstr. Surg. 96: 603, 1995.

45. Daane, S. P., and Rockwell, W. B. Analysis of methods for reporting severe and mortal lipoplasty complications. Aesthetic Plast. Surg. 1999; 23: 303.

46. Starling J, Thosani MK, Coldiron BM. Determining the Safety of Office-Based Surgery: What 10 Years of Florida Data and 6 Years of Alabama Data Reveal. Dermatologic Surgery. 2012; 38:2:171177.

47. Newman, L. Combination Cosmetic Surgeries, General Anesthesia Drive AEs. Medscape Medical News (C) 2012 WebMD, LLC. February 9, 2012.

48. Hanke CW. Commentary: Advocating for Mandatory Adverse Event Reporting. Dermatologic Surgery. 2012; 38:2:178-179.

49. Samdal, Frode; Amland, Petter F.; Bugge, Jan F. Plasma Lidocaine Levels During Suction-Assisted Lipectomy Using Large Doses of Dilute Lidocaine with Epinephrine. Plast. \& Reconst. Surg. 1994; 93(6):1217-1223.

50. Christman KD. Death following suction lipectomy and abdominoplasty (Letter). Plast. Reconstr. Surg. 1986; 78: 428.

51. Abbes $\mathrm{M}$ and Bourgeon Y. Fat embolism after dermatolipectomy and liposuction (Letter). Plast Reconstr Surg. 1989; 84: 546.

52. Brauman, D. Lipoplasty: A Case for a Low-volume Procedure. Aesthet Surg J. 2000; 20 (5): 373-379.

53. Liposuction Abdominoplasty: An Evolving Concept. Presented at the ASPS / PSEF / ASMS 71st annual scientific meeting November 2-6, 2002. San Antonio, Texas.

54. Brauman D. Liposuction abdominoplasty: An evolving concept. Plast Reconstr Surg. 2003; 112: 1: 288-298.

55. Brauman, D. Diastasis Recti: Clinical Anatomy. Plast. Reconstr. Surg. 2008; 122: 1564.

56. Brauman, D. Reply, letter to the editor. Diastasis Recti: Clinical Anatomy. Remus Repta, R., Hunstad, J.P. Plast. Reconstr. Surg. 2009; 123: 1885-1886.

57. Brauman, D. Reply, letter to the editor. Factors that May Influence Failure of the Correction of the Musculoaponeurotic Deformities of the Abdomen. Nahas, F. X., Barbosa, M. V., Ferreira, L.M. Plast. 
Reconstr. Surg. 2009; 124: 334-335.

58. Brauman D, Capocci J. Liposuction Abdominoplasty: An advanced Body Contouring Technique. Plast. Reconstr. Surg. 2009; 124(5):1685-1695.

59. Brauman D, van der Hulst RWJ and van der Lei B. A Case For Local Anesthesia and Prevention Protocols For Curbing DVT in Abdominoplasty.

60. Brauman D, van der Hulst RWJ and van der Lei B. Liposuction Assisted Abdominoplasty (LAA): An Enhanced Abdominoplasty Technique. Plastic and Reconstructive Surgery Global Open: September 2018 - Volume 6 - Issue 9 p e1940.

61. Brauman D, van der Hulst RWJ and van der Lei B. Abdominoplasty with Circumferential Liposuction, DVT, Local Anesthesia: How Much Proof is Needed. 2019; Plast Recnstr. Surg. In Press. 
Chapter 2.

Lipoplasty: A Case for a Low-volume Procedure

Chapter

\author{
Lipoplasty: \\ A Case for a Low-volume \\ Procedure
}

AESTHETIC SURGERY JOURNAL Volume 20

Number 5

October 2000 



\title{
Chapter 2.
}

\section{Lipoplasty: A Case for a Low-volume Procedure}

\author{
Aesthetic Surgery Journal: October 2000 - Volume 20, Number 5
}

Daniel Brauman, MBBS, MD

White Plains, N.Y

Background: During recent years, the maximum volume of wetting solution used in lipoplasty procedures has increased. However, an increase in reported deaths and complications from lipoplasty since the introduction of highvolume techniques have raised questions about the safety of these procedures.

Objective: This study evaluated the safety and effectiveness of low-volume lipoplasty performed with the patient under local anesthesia and conscious sedation.

Methods: A contour map indicating the volume of fluid infiltrated/fat to be removed from each elevation was drawn on the patient 1 to 2 days before the procedure. Low pressure was used to infiltrate subcutaneous fluid, first to the most superficial fat layer just deep to the skin and then, under higher pressure controlled by the surgeon, to the entire thickness of the fat layer. Fat removal commenced when skin vasoconstriction become evident. The total volume of aspirate removed per site was measured, and the fat content of the aspirate was estimated.

Results: In a series of 189 consecutive patients, the total amount of fat removed per procedure ranged from 246 to $4505 \mathrm{~mL}$ and averaged $1396 \mathrm{~mL}$. There were no major complications.

Conclusions: It is possible to perform lipoplasty effectively on obese patients and/or patients requiring treatment of a large percentage of body surface area while using less than $5 \mathrm{~L}$ of subcutaneous infiltration. Low-volume infiltration enables extensive fat removal with potentially less risk and fewer complications than higher-volume infiltration techniques.

In the early 1980s, lipoplasty became established as a safe, effective, and relatively simple procedure. During the past few years, the upper limit of the infiltrated fluid volume, beyond which liposuction is considered to be a "high-volume" procedure, has increased. ${ }^{1-5}$ Reported deaths and complications, especially those related to "high- volume" lipoplasty, have raised questions about its safety. ${ }^{6-12}$ In an effort to reduce the number of lipoplasty complications, the American Society for Aesthetic Plastic Surgery, the American Society of Plastic Surgeons, and other organizations have called for more stringent guidelines. ${ }^{8,9,11-13}$ These include guidelines for patient selection, facility accreditation, intraoperative monitoring (such as temperature probes and Foley catheters), fluid management, formulas, and monitored overnight stays. 
Table 1. Volumes of fat removed per area in 158 patients

\begin{tabular}{|c|c|c|c|}
\hline Site & $\begin{array}{l}\text { No. of sites treated } \\
\text { in } 158 \text { cases }\end{array}$ & $\begin{array}{l}\text { Average volume of fluid } \\
\text { infiltrated/fat removed } \\
\text { per site }(\mathrm{mL})\end{array}$ & $\begin{array}{l}\text { Range of volumes of } \\
\text { fat removed per site }(\mathrm{mL})\end{array}$ \\
\hline Breast (men only) & 38 & 210 & $52-605$ \\
\hline Abdomen & 107 & 496 & $10-2400$ \\
\hline $\begin{array}{l}\text { Hip, adjacent back, and } \\
\text { buttock }\end{array}$ & 200 & 246 & $30-1000$ \\
\hline Back and waist & 154 & 236 & $16-840$ \\
\hline Lateral thigh & 200 & 243 & 10-1095 \\
\hline Anterior thigh & 50 & 137 & $35-380$ \\
\hline Inner thigh & 92 & 96 & $30-520$ \\
\hline Knee & 76 & 41 & $10-150$ \\
\hline Calf & 12 & 101 & $9-200$ \\
\hline Arm & 14 & 90 & $15-150$ \\
\hline Neck & 2 & 7.5 & $5-10$ \\
\hline
\end{tabular}

In this article, I demonstrate that it is possible to safely and effectively perform extensive lipoplasty through use of a low-volume infiltration. Recommendations regarding anesthesia and fluid management are suggested to ensure a simplified and safe procedure. I propose a revised classification of lipoplasty techniques based on infiltration volume rather than aspiration volume. Finally, I outline a method for a reproducible estimate of the volume of fat removed. ${ }^{1}$

\section{Materials and Methods}

One hundred eighty-nine consecutive patients between the ages of 15 and 66 years were operated on and the charts retrospectively reviewed. The average patient age was 40.5 years; $51 \%$ of patients were older than 40 years and $21 \%$ were older than 50 years. One hundred fiftyeight of the patients (84\%) were women and $31(16 \%)$ men.

The patients' average weight was $159.47 \mathrm{lb}$; each of 26 patients (14\%) weighed more than 200 lb. Associated medical conditions included 20 cases of hypertension, 13 cases of cardiac history, 6 cases of asthma, 9 cases of thyroid disease, and 3 cases of diabetes. These data reflect the fact that most of the patients were aged 40 years or older.

The body sites treated included the breast (in male patients only), abdomen, hip (including adjacent back and buttock), waist and back (including the upper back), lateral thigh, anterior thigh, medial thigh, knee, calf, arm, and neck.

The ratio of the wetting solution infiltrated to the volume of fat removed was 1:1. (This ratio reflects a calculation of the volume of fat based on the total aspirate). During the procedure, the 
total volume of fluid removed (aspirate), per site, was calculated by tallying the contents of all the syringes.

The aspirated fluid of each area or group of similar areas was allowed to settle in transparent containers for approximately 30 minutes. If no more than $10 \%$ of the aspirate was wetting solution, the volume of fat removed per area (fat content of aspirate) was considered to be equal to the volume of the aspirate. If the aspirate contained more than $10 \%$ wetting solution, the volume of fat removed was determined by subtracting the volume of the excess wetting solution from the volume of the total aspirate.

Because the volume of the instilled fluid was low and because it was introduced into multiple tunnels created in the fat layer with syringes under low pressure, the aspirate typically contained no more than $10 \%$ wetting solution and therefore approximated the volume of the infiltration fluid.

The total volume of fat removed per procedure averaged $1396 \mathrm{~mL}$ and ranged from 246 to 4505 $\mathrm{mL}$. Information regarding the exact volume of wetting solution introduced/fat removed per area is available on 158 of the 189 patients treated (Table 1). The number of areas treated during a single procedure ranged from 3 to 15 , the average being 7 . Thirty-eight patients (24\%) had 10 or more areas treated. Eight patients underwent concurrent abdominal skin excision in addition to liposuction. The average follow-up time was 7.29 months.

\section{Operative Technique}

A contour map was drawn on the patient 1 to 2 days before surgery, the goal being to exactly define the elevations and depressions of each area to be treated. The volume of fluid infiltrated/fat to be removed from each area was estimated and marked on the contour map. These amounts, when added, equaled the total amount of fluid infiltrated/fat removed and totaled less than $5 \mathrm{~L}$ in every case.

The volume of fluid infiltrated equaled the estimated volume of fat that was aspirated. The amounts of fluid infiltrated in areas where the skin/tissue is exceptionally loose may exceed the estimated amounts of fat to be removed by up to $30 \%$. This excess stabilizes the tissue and eases the performance of lipoplasty but has no effect on hemostasis or anesthesia. No tumescence is desired. Surgery is performed through use of the syringe technique, and the typical amounts of fat aspirated are noted in the Table. 


\section{Low Pressure/Volume Anesthesia}

Each patient was premedicated with meperidine $10 \mathrm{mg} / \mathrm{kg} \mathrm{IM}$ and hydroxyzine $50 \mathrm{mg} \mathrm{IM}$ and kept sedated with intravenous meperidine titrated in increments of 5 to $10 \mathrm{mg} / \mathrm{dose}$ and intravenous midazolam titrated in increments of 0.2 to $0.4 \mathrm{mg} /$ dose. Patients were continuously monitored in accordance with accepted anesthesia standards.

A.

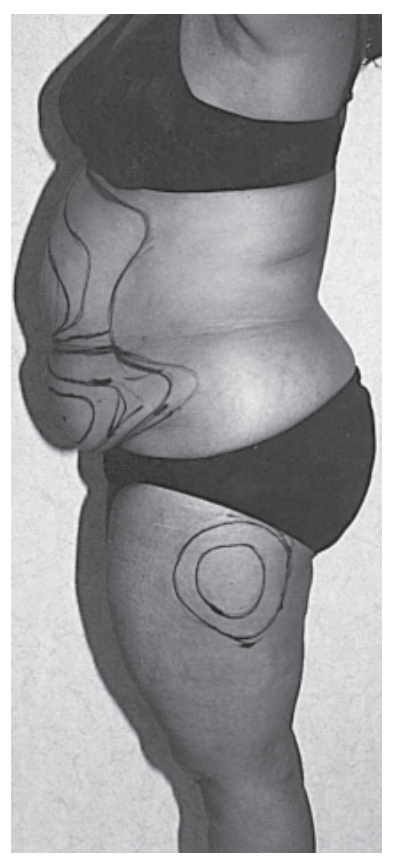

B.

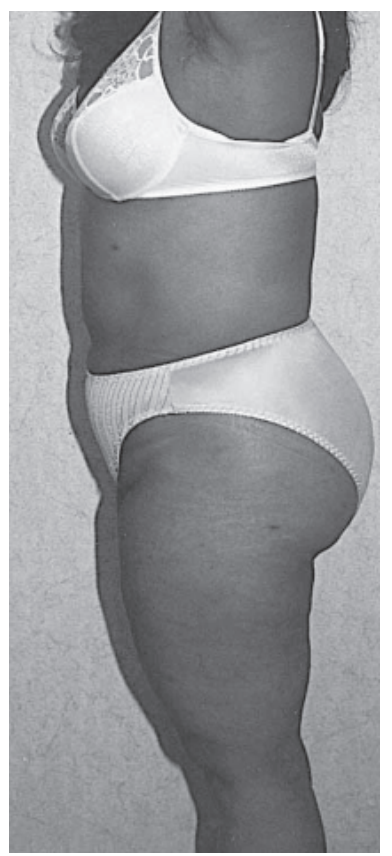

Fig. 1. A, Preoperative view of a 41-year-old woman shows body contour mapping.

B, Post-operative view 7 months after $1048 \mathrm{~mL}$ of fat were removed from the abdomen.

\section{The Low Pressure Administration Technique}

Most patients do not tolerate the instillation of a large volume of dilute anesthetic fluid without prior anesthesia of the more sensitive skin. A 2-phase method of administration of the wetting fluid enables performance of the procedure with the patient under conscious sedation. In the first phase, infiltration of the skin and subdermal fat is performed by injecting $0.25 \%$ lidocaine with 1:400,000 of epinephrine by means of $10-\mathrm{mL}$ syringes with $25 \mathrm{G}$ spinal needles. This slow, low-pressure fluid infiltration provides anesthesia to the skin and to the superficial subcutaneous 
tissue. More local anesthetic is injected into areas where the cutaneous nerves are known to exit the deep fascia (eg, near the midline of the back at the location where the cutaneous branches of the dorsal rami of the spinal nerves exit the deep fascia). This provides a nerve block effect that both improves the anesthesia to the skin and provides some anesthesia for the entire depth of the subcutaneous tissue. The skin remains soft and supple because of the very low volume of the anesthetic fluid used. The local anesthetic is administered slowly. The maximum amount of $0.25 \%$ lidocaine with epinephrine infiltrated in a 1-hour period is $120 \mathrm{~mL}$ ( 2.5 to $4.28 \mathrm{mg} / \mathrm{kg}$ ).

In the second phase, infiltration of the entire thickness of the fat layer with a $0.056 \%$ lidocaine solution with epinephrine is performed through use of $60-\mathrm{mL}$ Toomey syringes with $3-\mathrm{mm}$ infiltration cannulas. The fluid is instilled into multiple tunnels and the amount of pressure is varied and controlled by the surgeon according to the patient's tolerance. Although this instillation is faster and at higher pressure than the first phase, it is still a low- pressure, low-rate procedure in comparison with instillation by means of commercially available infusion devices. ${ }^{2,4,14,15}$ Low-rate fluid infiltration is performed in a gradual, piecemeal fashion.

A.

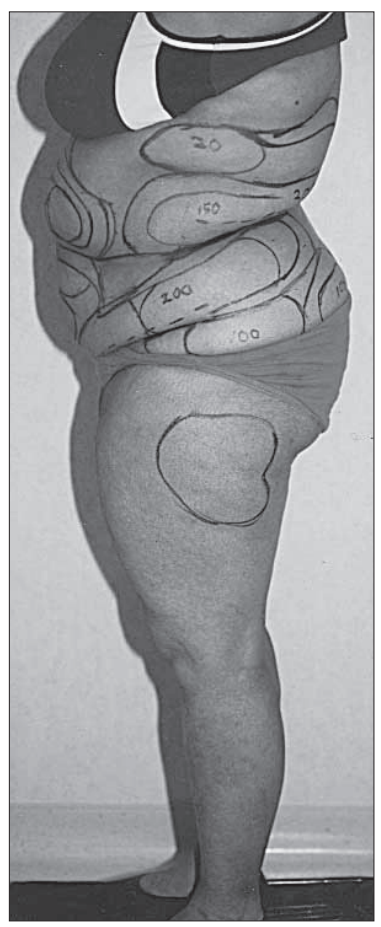

B.

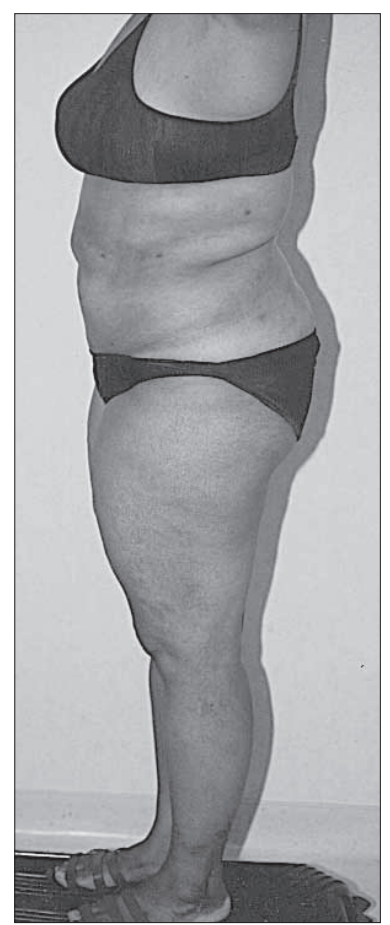

Fig. 2. A, Preoperative view of a 44-year-old woman shows volume (in milliliters) of fluid infiltrated/fat to be removed marked on body contour map. B, Postoperative view 4 months after surgery in which $2460 \mathrm{~mL}$ of fat were removed. 
Each area or group of areas is first infiltrated and then immediately treated. The maximum total dose of lidocaine infiltrated per hour $(0.25 \%$ and $0.056 \%)$ is $10 \mathrm{mg} / \mathrm{kg}$. The Physician's Desk Reference allows a maximum dose of $7 \mathrm{mg} / \mathrm{kg}$ of $1 \%$ lidocaine with epinephrine when infiltrated. ${ }^{16}$

Anesthetization of the skin (which is much more sensitive than the fat layer) nerve blocks, and then separate anesthetization of the fat layer with fluid under low pressure allow performance of this procedure both under conscious sedation and with less fluid Less fluid is required because tissue tumescence, which by itself can provide a certain degree of anesthesia and vasoconstriction, is unnecessary.

Instead, the necessary vasoconstriction is obtained through the use of modest amounts of epinephrine. When skin vasoconstriction becomes evident, local anesthesia has been established and fat removal can begin. The total amount of lidocaine used never exceeds $35 \mathrm{mg} / \mathrm{kg}$ and is pre-calculated for each patient. ${ }^{17}$

A solution of $0.056 \%$ lidocaine is prepared by mixing $60 \mathrm{~mL}$ of $1 \%$ lidocaine with $1: 100,000$ of epinephrine with $1000 \mathrm{~mL}$ of normal saline for injection. Epinephrine $(1 \mathrm{mg})$ may be added to this solution. Typically, in a patient weighing $154 \mathrm{lb}$, a total of $240 \mathrm{~mL}$ of $0.25 \%$ lidocaine with epinephrine is injected, along with an additional $3 \mathrm{~L}$ of $0.056 \%$ lidocaine to be infiltrated. In a patient weighing $264 \mathrm{lb}, 360 \mathrm{~mL}$ of $0.25 \%$ lidocaine with epinephrine is typically used, along with as much as $5 \mathrm{~L}$ of $0.056 \%$ fluid. In general, the more $0.25 \%$ lidocaine with epinephrine that is used in the first phase, the less $0.056 \%$ lidocaine solution that is necessary in the second phase. The larger the person, the easier it is to allocate the fluids, in as much as more lidocaine can be used.

On completion of surgery, warmed fluid hydration is performed. No specific fluid hydration formula is used. The total amount of intravenous fluids for the day is determined clinically according to the total body surface area treated, the length of the procedure, and urine output.

Most commonly, $3 \mathrm{~L}$ of crystalloid satisfy the above criteria. If the procedure is more extensive or if the patient has been NPO for a longer length of time than usual, $4 \mathrm{~L}$ may be required. At discharge, family members are instructed to ambulate the patient once an hour every 4 to 6 hours, and patients are instructed to periodically flex and extend their feet while they are in bed. 


\section{Results}

Figures 1 and 2 demonstrate typical cases in which the low- volume technique was used. The modest amounts of fluid infiltrated/fat removed demonstrate that it is possible to perform adequate fat removal even in obese individuals by using a low-volume technique. Figure 3 demonstrates the thick and pasty nature of the fat that is removed by this technique. Figure 4 demonstrates a typical sample of "thick" fat removed together with a sample of the same fat that was spun down for 20 minutes.
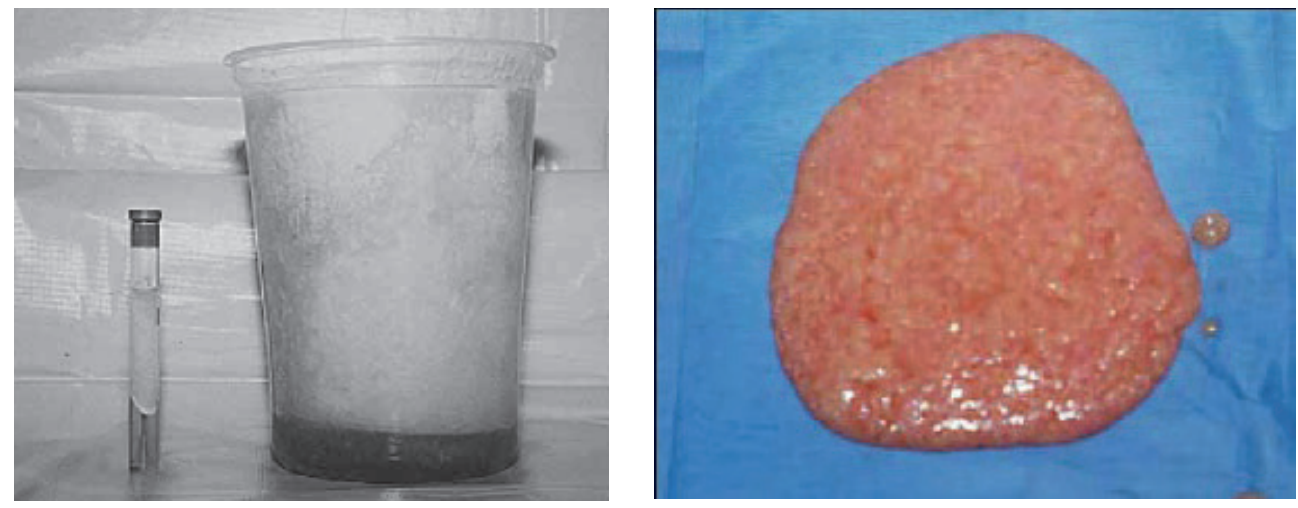

Fig. 3. Fat removed during low-volume lipoplasty had a thick, pasty consistency. When submitted for microscopic analysis, it was reported as normal fat.

Fig. 4. A container with a sample of fat removed by the low-volume technique and allowed to settle for 5 hours. Next to it is a tube with the same specimen spun down for 20 minutes.

There were no major complications, such as hematomas, seromas, or nerve problems. Five patients, including 3 African-American patients, had significant post-inflammatory hyperpigmentation that resolved within 1 year. In 1 patient who underwent a concurrent abdominal skin excision (not abdominoplasty), a minor wound infection developed; it responded to drainage. Ten patients subsequently had additional lipoplasty in the same areas, in additional areas, or in both. Five patients had revisions of mostly small persistent lateral fat deposits. All patients were discharged on the day of surgery.

\section{Discussion}

Today, almost all lipoplasty procedures are performed through use of wet techniques. The most common classification of lipoplasty procedures describes 4 such techniques. The dry technique is defined as one in which no fluid is infiltrated. In the wet technique, 200 to $300 \mathrm{~mL}$ of fluid is 
infiltrated per area. The superwet technique is defined as a ratio, as follows: infiltrate/aspirate = $1: 1^{2,17}$

The tumescent technique is described both as achievement of skin turgor ${ }^{2,14}$ and as a ratio, as follows: infiltrate/aspirate $=2-8: 1 .^{2,18,19}$ In other words, the dry technique and the wet technique are defined as volumes, the superwet technique as a ratio, and the tumescent technique as both an endpoint and a ratio.

There are several problems with this classification. The definition of the superwet technique is too broad. In a given patient, within a given treatment site, 2 surgeons performing the superwet technique might instill and remove vastly different amounts of fluid. There are also significant overlaps among the various techniques. For example, in a small area, $300 \mathrm{~mL}$ of instilled fluid might be sufficient to produce tissue turgor (tumescence), whereas if $300 \mathrm{~mL}$ of aspirate rich in fat were to be removed, the 1:1 ratio defining the superwet technique would be achieved. Most commonly, the tumescent and superwet techniques overlap, and surgeons use the 2 terms interchangeably. ${ }^{2,18,19}$ The reason for this is that defining the use of fluid as a ratio allows a very large range of fluid volumes to be included. In addition, the denominator of this ratio (the aspirate) does not satisfactorily fulfill one of the objectives of a classification system because it does not accurately describe the volume of fat that is actually removed. ${ }^{1}$

A review of the literature shows that surgeons vary widely in their reports of the amount of aspirate removed in similar cases. ${ }^{3,5,20-27}$ The estimate of the volume of fat that makes up any given area varies greatly from surgeon to surgeon and is entirely subjective. The only objective way to find out the precise volume of excess fat is to excise the tissue and weigh it. Moreover, the "drier" the extraction technique, the more closely the volume of aspirate will approximate the true volume of fat in that area. With these considerations in mind, I suggest the following classification, which is based on volumes rather than ratios:

- Volume of fluid infiltrated (wetting solution, infiltrate):

Less than $5 \mathrm{~L}$ : low volume, or $<70 \mathrm{~mL} / \mathrm{kg}$

Five to $15 \mathrm{~L}$ : high volume, or 70 to $210 \mathrm{~mL} / \mathrm{kg}$ More than $15 \mathrm{~L}$ : higher volume, or >210 $\mathrm{mL} / \mathrm{kg}$

- Volume of fat removed (fat content of aspirate):

Volume of the visible column of fat (Allow the total amount of aspirate to settle for 2 hours, and/or allow the amount of aspirate per area to settle for 30 minutes.) 
The volume of the wetting solution, together with the volume of the intravenous fluid administered, represents the total fluid load and accurately describes the extent of the procedure. In most cases, the total volume of the aspirate is equal to or smaller than the total volume of the infiltrate (even with the low-volume technique). Therefore, it is not an accurate measure of fluid load and physiologic stress. The volume of fat removed (fat content of aspirate) has been determined arbitrarily for the sake of convenience and uniformity. Intraoperatively, the aspirate settles for 30 minutes only; this avoids clutter and multiple containers on the nurse's table and yet provides a fairly accurate estimate. Postoperatively, allowing the aspirate to settle for more than 3 hours does not seem to significantly alter the visible amount of fat. A more accurate method for research purposes would be to spin down a sample of aspirate for 20 minutes and determine its content of fat particles, salt-water solution, serum, "oil," and red cells. Sample analysis by electrophoresis is also a possibility.

Classifications are useful and ensure that different surgeons communicate using the same "language."

However, they may also impose standards that may hamper free thought, scientific debate, and innovation. In addition, they may have medico-legal implications; for instance, dubbing a technique "high volume" may translate legally as too much fluid. The author believes that a classification should be all-inclusive and should not take the place of scientific debate.

Different lipoplasty techniques produce different compositions of aspirated fat. The syringe technique produces the least amount of vaporization and emulsification. Many samples were submitted for microscopic analysis and reported as normal fat. The aspirating machine produces more emulsification and vaporization, whereas ultrasound-assisted lipoplasty produces the greatest breakdown of the aspirated fat.

\section{High-volume Verses Low-volume Lipoplasty}

The average intravascular volume in a 154-lb person is $5 \mathrm{~L}$. In high-volume lipoplasty, more than $5 \mathrm{~L}$ of fluid are infiltrated. Even though most healthy patients can tolerate massive infusions, the physiologic adaptations of all organ systems are stressed by such infusions, and despite the warming of the fluids, a certain degree of hypothermia may result. This is especially true for obese patients who are already physiologically stressed. In many of these patients, the respiratory system is always problematic, and some of them may exhibit somnolence secondary to obesityrelated hypoventilation (the Pickwickian syndrome). Merely sedating these patients will drop their oxygen saturation to approximately $85 \%$ to $90 \%$. They are also more prone to deep vein thrombosis, cardiac problems, and hypertension. Even if they are asymptomatic, obese patients are at a higher risk for surgery. Yet these are the very patients in whom high-volume lipoplasty is performed. ${ }^{5,19,20,24,25,27}$ 
The low-volume technique is simply a drier technique that is both safe and effective. The amount of fat removed per area is similar to the amounts of fat removed through use of dry techniques. ${ }^{28}$ Moreover, this technique makes it possible to perform lipoplasty effectively on obese patients and/or patients with a large percentage of their body surface area to be treated with less than $5 \mathrm{~L}$ of fluid infiltrate. These are the patients on whom high- volume lipoplasty is presently performed to achieve similar results. ${ }^{4}$

The goals of fluid infiltration in lipoplasty-hemostasis, anesthesia, and ease of dissection-are easily achieved through use of the low-volume technique described here. The addition of lidocaine and epinephrine provides an even higher degree of anesthesia and vasoconstriction.

Effective hemostasis does not require tumescence. The addition of $1 \mathrm{mg}$ of epinephrine to the infiltration fluid produces effective vasoconstriction in the subcutaneous tissue. Even smaller concentrations of epinephrine also provide excellent hemostasis in many cases. .,20,21,26 $^{2}$

Many plastic surgeons perform lipoplasty with the patient under general anesthesia, because high-pressure/volume fluid infiltration is a painful process. ${ }^{2}$ Staging of nerve blocks and skin anesthesia, followed by anesthesia of the fat layer and low-pressure manual infiltration of the anesthetic fluid, permits excellent regional anesthesia. The ability to treat large body surface areas with sedation attests to the effectiveness of this technique.

Dissection is easier when some fluid is instilled. Tissue tumescence minimally increases the ease of dissection but may reduce the ability of the surgeon to "feel" the characteristic resistance of the various tissue layers. This may contribute to abdominal perforations, especially when combined with the muscle relaxation of general anesthesia. ${ }^{6,7,29,30}$

\section{Conclusion}

This article describes a low-volume lipoplasty technique and defines it by detailing both the range and the average amounts of fluid infiltrated into the various lipoplasty areas. Reduction of the fluid load lowers the level of physiologic stress to which the body is subjected, especially when an obese patient is being treated and/or a large percentage of the body surface area is involved. Low-volume lipoplasty enables extensive fat removal with potentially less risk and fewer complications than higher-volume lipoplasty, based on my own experience with high-volume lipoplasty of up to $9 \mathrm{~L}$ of infiltrated fluid.

In addition to the 189 cases summarized here, I have performed several hundred additional procedures using the same technique with good results and no complications. 
The low-pressure anesthetic technique described in this article permits the performance of lipoplasty with the patient under conscious sedation. Furthermore, local anesthesia eliminates the added risks of general anesthesia, which together with blood clots are associated with most reported deaths associated with lipoplasty. ${ }^{23}$ This technique can also be used for many other plastic surgical procedures, including breast reductions, sub- muscular breast augmentations, and abdominal dermolipectomies.

\section{References}

1. Matarasso A. Superwet anesthesia redefines large-volume liposuction. Aesthetic Surg J 1997;17:358-364.

2. Rohrich RJ, Beran SJ, Fodor PB. The role of subcutaneous infiltration in suction-assisted lipoplasty: a review. Plast Reconstr Surg 1997;99:514- 519.

3. Albin RE. Risks and possible limits of large volume liposuction. Presented at the 67th Annual Meeting of the ASPRS; 1998 October; Boston, MA.

4. Matarasso A. Lidocaine in ultrasound-assisted lipoplasty. Clin Plast Surg 1999;26:431-439.

5. Peren PA, Gomez JB, Guerrero-Santos J. Total corporal contouring with megaliposuction. Aesthetic Plast Surg 1999;23:93-100.

6. Rohrich RJ, Beran SJ. Is liposuction safe? Plast Reconstr Surg 1999; 104:819-822.

7. Grazer FM, DeJong RH. Fatal outcomes from liposuction: Census Survey of Cosmetic Surgeons. Plast Reconstr Surg 2000;105:436-446.

8. ASPRS urges members to exercise caution in lipoplasty procedures; task force report calls for scrutiny of training, large volume removals. PS News Bulletin January 26, 1998.

9. California committee calls for restriction of large volume lipoplasty to hospitals. Plast Surg News August 1998.

10. Gorney M. Suction assisted lipoplasty: complications and fatal out- comes. Presented at the 67th Annual Meeting of the ASPRS. Boston, MA; 1998 October.

11. Del Junco R. Plastic/cosmetic surgery committee of the Medical Board of California. AAAASF News 1999;6:1.

12. Rao RB. Deaths related to liposuction. New Engl J Med 1999;340:1471.

13. Mathes SJ. PSEF lipoplasty research task force seeks data. Plast Reconstr Surg 1998 (suppl).

14. Klein JA. The tumescent technique. Dermatol Clin 1990;8:425. 
15. Hunstad JP. The tumescent technique: a safe and efficacious method to minimize blood loss and provide conscious anesthesia for liposuction procedures. Presented at the 27 th Annual Meeting of ASAPS; 1994 April 17; Dallas, TX.

16. Physician's Desk Reference. 54th ed. Montvale, NJ: Medical Economics Company; 2000:638.

17. Klein JA. Tumescent technique for regional anesthesia permits lido- caine doses of $35 \mathrm{mg} / \mathrm{kg}$ for liposuction. Dermatol Surg Oncol 1990; 16:248-263.

18. Fodor PB. Wetting solutions in aspirative lipoplasty: a plea for safety in liposuction. Aesthetic Plast Surg 1995;19:379-380.

19. Fodor PB. Lipoplasty: another plea for safety! Aesthetic Plast Surg 1998;22:399.

20. Klein JA. Tumescent technique for local anesthesia improves safety in large-volume liposuction. Plast Reconstr Surg 1993;92;1085-1098.

21. Amland PF, Bugge JF. Blood loss during liposuction using the tumescent technique. Aesthetic Plast Surg 1994;18:157-160.

22. Hunstad JP. Tumescent and syringe liposculpture: a logical partnership. Aesthetic Plast Surg 1995;19:321-333.

23. Pitman GH, Aker JS, Tripp ZD. Tumescent liposuction: a surgeon's per- spective. Clin Plast Surg 1996;23:633-641; discussion, 642-645.

24. Cardenas-Camarena L, Gonzalez LE. Large-volume liposuction and extensive abdominoplasty: a feasible alternative for improving body shape. Plast Reconstr Surg 1998;102:1698-1707.

25. Albin R, de Campo T. Large-volume liposuction in 181 patients. Aesthetic Plast Surg 1999;23:5-15.

26. Troilius C. Ultrasound-assisted lipoplasty: is it really safe? Aesthetic Plast Surg 1999;23:307-311.

27. Cardenas-Camarena L, Tobar-Losada A. Large-volume circumferential liposuction with tumescent technique: a sure and viable procedure. Plast Recontr Surg 1999;104:1887-1899.

28. Herhahn FT. Physical evaluation and informed consent. In: Hetter GP, ed. Lipoplasty. Boston/Toronto: Little, Brown \& Co.; 1984:95-104.

29. Gilliland MD. Abdominal perforations. Presented at the 67th Annual meeting of the ASPRS, Boston, MA.

30. Gilliland MD, Commons GW, Halperin B. Safety issues in ultrasound- assisted large-volume lipoplasty. Clin Plast Surg 1999;26:317-335. 
Chapter 3.

Liposuction Abdominoplasty: An Evolving Concept

Chapter

\section{Liposuction Abdominoplasty: An Evolving Concept}

Plastic and Reconstructive Surgery VOLUME 112 Issue 1 p $288-298$ July 2003 



\title{
Chapter 3.
}

\section{Liposuction Abdominoplasty: An Evolving Concept}

\author{
Plastic and Reconstructive Surgery: July 2003 - Volume 112 - Issue 1p 288-298
}

\author{
Daniel Brauman, MBBS, MD
}

\section{White Plains, N.Y}

From the Department of Plastic Surgery, Cornell University, Weill Medical College. Received for publication March 22, 2002; revised September 30, 2002.

\begin{abstract}
Liposuction abdominoplasty-liposuction of abdominal subcutaneous tissue deep and superficial to Scarpa's fascia, with excision of excess abdominal skin and, when indicated, plication of the anterior rectus sheath without undermining -is an effective, low-risk approach to minimizing abdominal flap undermining. The technique allows aggressive thinning and "sculpting" of full-thickness abdominal subcutaneous tissue and achieves a natural (not featureless) abdominal contour. It minimizes the creation of "dead space," which often leads to postoperative complications, as well as preserves sensory nerve and blood supply to the abdominal skin. The operation may be performed with the patient under local anesthesia, which probably diminishes the risk for deep vein thrombosis. Moreover, additional procedures can be conducted safely, and the postoperative course is short, uneventful, and without restrictions; patients return to normal activity within a week or so. New evaluation criteria for abdominoplasty are discussed in this article, the most important of which is the assessment of intraabdominal fat content and its impact on surgical outcome and the decision to perform anterior rectus sheath plication. The concept of a sliding, mobile, sensate abdominal flap, created by liposuction and sustained by multiple neurovascular mesenteries, is also offered.
\end{abstract}

Traditional abdominoplasty is associated with the potential for several problems. The procedure has been identified as a specific risk factor for deep vein thrombosis by Daane and Rockwell ${ }^{1}$, Reinisch et al., ${ }^{2}$ and Stuzin et al. ${ }^{3}$ Dillerud ${ }^{4}$ found that wide undermining causes skin necrosis. In addition, undermining necessitates prolonged postoperative suction drainage to avoid seroma, which is the most frequent complication of abdominoplasty. ${ }^{5-10}$ It has also been reported by van Uchelen et al. ${ }^{11}$ to produce sensory changes of the abdomen and thigh. Further, incisions are often long and geometrically designed, and tension may cause excessive scarring. ${ }^{7-9}$ In contrast, limited abdominal flap undermining, which has resulted in less morbidity, fewer complications, and shorter scars than the classic procedure, has been advocated for appropriate cases by Wilkinson and Swartz, ${ }^{12,13}$ Greminger, ${ }^{14}$ Eaves et al., ${ }^{15}$ Zukowski et al., ${ }^{16}$ Shestak, ${ }^{17}$ and Lockwood. ${ }^{18,19 .}$ 
In this article, liposuction abdominoplasty is proposed as a safe and highly effective alternative to traditional abdominoplasty in appropriate patients. In the author's clinical experience, the procedure has consistently and significantly reduced many of the problems that have been associated with classic abdominoplasty. ${ }^{1-11}$ Other related concepts are also offered: The successful outcome of abdominal wall surgery appears to depend on the preoperative evaluation of the intraabdominal fat content, and a sliding abdominal flap, created by liposuction, is mobile and has unique advantages in comparison with undermining.

\section{Patients and Methods}

\section{Patients}

For the 43 patients who underwent liposuction abdominoplasties beginning in 1997 (group III), a total of 60 consecutive patients, seen between 1997 and 2002, are presented. Four underwent traditional abdominoplasties (group I), 15 had traditional abdominoplasties performed elsewhere (group II), and 43 underwent liposuction abdominoplasties (group III). A rectus sheath plication (xiphoid to pubis) was performed in the four patients of group I, the 15 patients of group II, and in four of the patients in group III (a total of 23 patients).

Of the 15 group II patients, two subsequently underwent liposuction abdominoplasty and were therefore also included in group III. Eleven patients subsequently underwent liposuction and two were evaluated in consultation only.

The patients in group III (40 women and 3 men) represent 7 percent of the approximately 580 major liposuction cases performed by the author during the same 5 -year period. They ranged in age from 17 to 64 years; their weight was between 111 and 252 pounds (average weight, 163.65 pounds; body mass index, 20.95 to 41.24; average body mass index, 28.53). Nineteen of these patients (44 percent) had hanging panniculi; among them, nine panniculi were first liposuctioned and then excised, which enabled a rapid and bloodless removal. The weights of the nine panniculi (which excluded the liposuctioned portion) ranged from 220 to $3057 \mathrm{~g}$ (average weight, $1158 \mathrm{~g}$ ). The amount of fat liposuctioned from the abdomen ranged from 50 to $7450 \mathrm{cc}$ (average amount, $3062 \mathrm{cc}$ ). The fat was liposuctioned using the syringe technique and allowed to separate for approximately 2 to 3 hours so accurate measurements could be obtained. Moreover, 38 patients from this group underwent liposuction of additional body areas. The total amount of liposuctioned fat per procedure ranged from 500 to $7800 \mathrm{cc}$ (average amount per procedure, $3315 \mathrm{cc})$. 


\section{Operative Technique}

Operations were performed with the patients under local anesthesia with sedation. Patients were premedicated with meperidine $10 \mathrm{mg} / \mathrm{kg}$ and hydroxyzine $50 \mathrm{mg}$ intramuscularly and kept sedated with intravenous meperidine, midazolam, and propofol. A low-pressure/low-volume technique was used. ${ }^{20}$ At first, nerve blocks were obtained by injecting $0.25 \%$ lidocaine with 1:400,000 epinephrine. Next, diluted anesthetic fluid, consisting of $60 \mathrm{ml}$ of $1 \%$ lidocaine and epinephrine with $1 \mathrm{mg}$ of epinephrine and $1000 \mathrm{ml}$ of normal saline, was infiltrated slowly under low pressure, using 60-ml Toomey syringes. ${ }^{21-28}$

Liposuction was performed with the usual aesthetic and safety considerations in mind. Most importantly, the subdermal blood supply must be preserved, and the creation of large dead spaces must be avoided (Fig. 1, below). These safety considerations do not apply to the panniculus because its skin is subsequently excised. Next, a curvilinear incision along the lower abdominal crease was performed. The incision did not extend past the iliac spines unless a large hanging panniculus required excision. The skin was bluntly detached and excised, and care was taken not to undermine the inferior border of the remaining abdominal skin (Fig. 2, below).

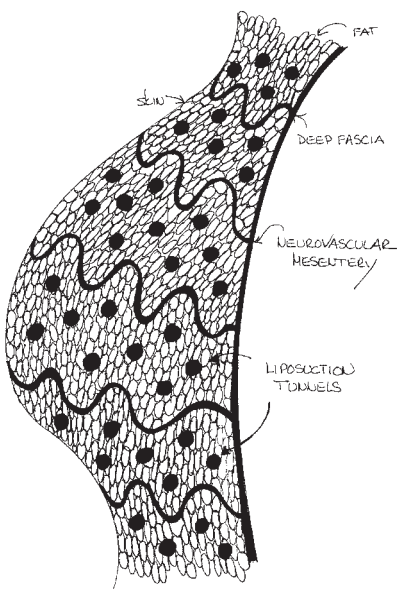

a

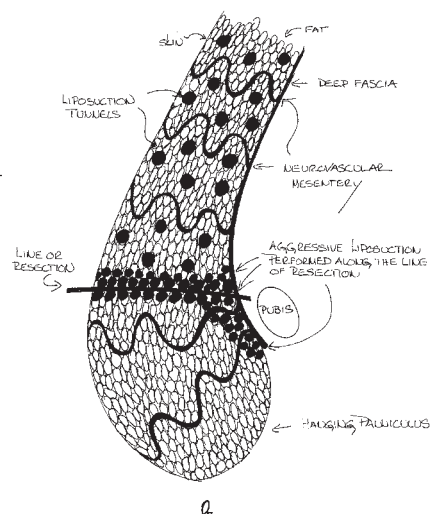

Q.

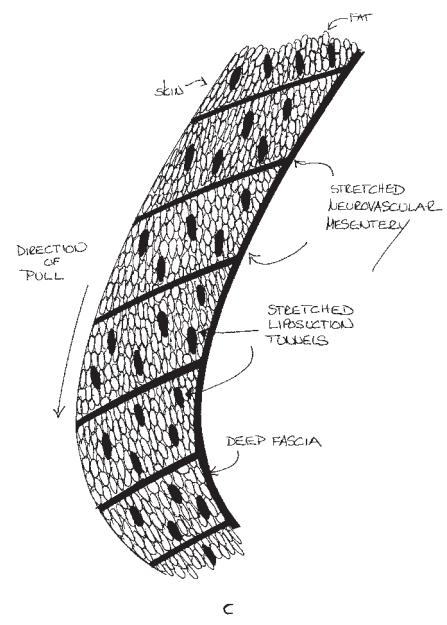

c

FIG. 1. Sagittal section. (Left) Liposuction (closed) of the entire subcutaneous fat layer; creation of the sliding flap. (Center) Liposuction (closed) deep along the abdominal wall (deep fascia) and along the line of resection enables separation of the hanging panniculus without blood loss from the abdominal wall and subcutaneous tissue superiorly. (Right) The sliding flap has been stretched inferiorly. Mobility is enabled by fat removal, transection of tethering fibers, and stretching of neurovascular mesenteries. 

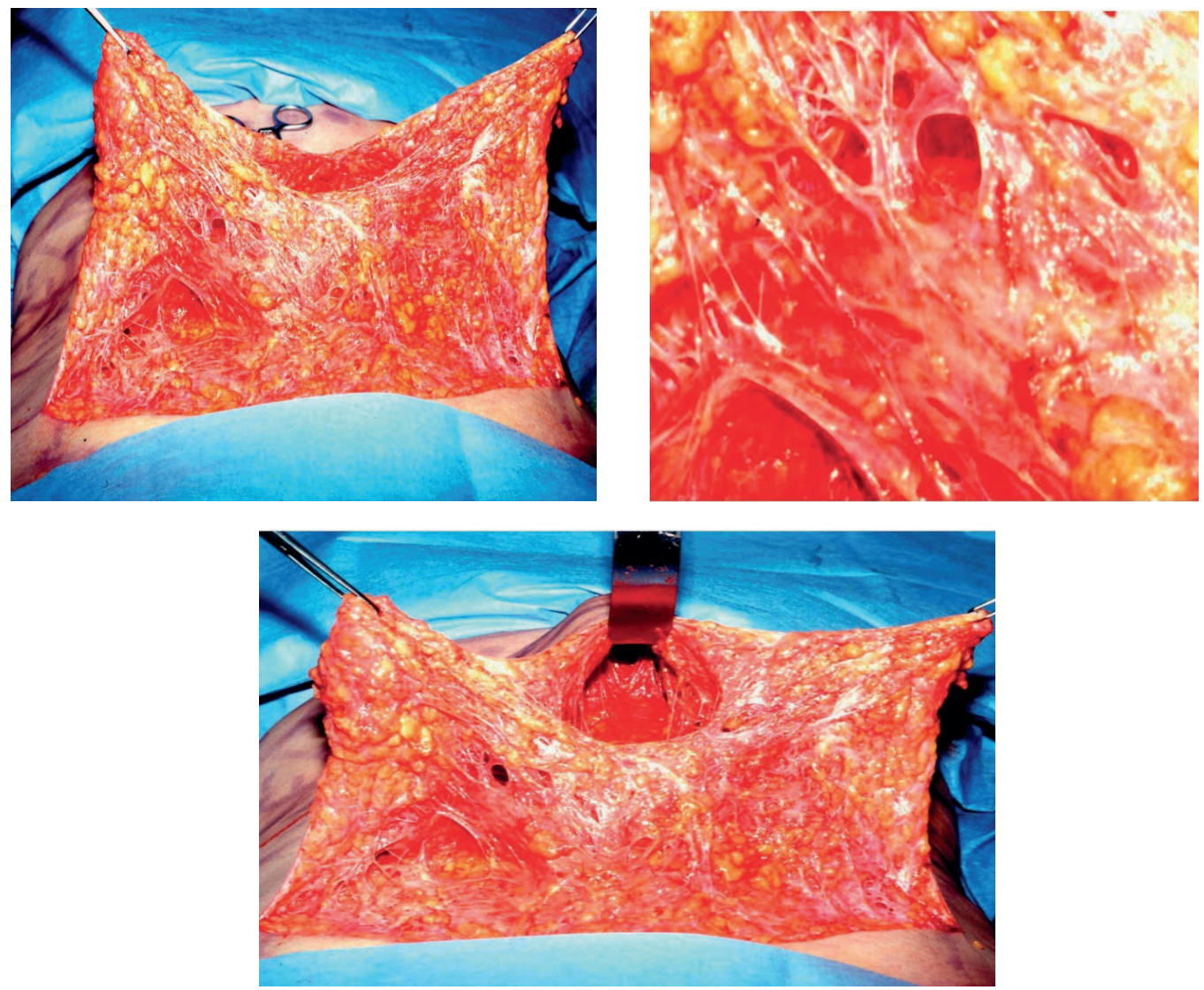

FIG. 2. The liposuction sliding flap with the infraumbilical panniculus still attached and split in the midline. The umbilical opening is seen in the center of the upper border. (right) Magnified view of the mesentery. Blood vessels and nerves are held together by a thin veil of fibrous tissue. (Center) The same flap (as viewed through the circular umbilical opening). Note the range of flap mobility and exposure for diastasis repair (through the umbilical opening).

Through a circular periumbilical incision, the retaining fibrous midline connections of the infraumbilical and supraumbilical abdominal skin were detached to gain flap mobility ( $\underline{\text { Fig. } 2}$, above). A rectus sheath plication was then performed in appropriate patients (Fig. 3, below). A neoumbilicus was formed superior to the old umbilical site, which was repaired vertically or incorporated into the horizontal incision. A two-layer closure, under some tension, was completed, and four 1-inch long, 1/4-inch-thick Penrose drains were stapled into the incision. Postoperatively, 3 liters of crystalloid usually produced a sustained urinary output; on occasion, 4 liters may have been required. All patients were seen the morning after the operation in a 
follow-up visit, and their Penrose drains were removed. However, in large-volume cases, two Penrose drains were left in place for an additional 2 days. Patients were encouraged to resume their normal activities without limitations. Strenuous exercise was allowed as soon as the patient was ready, usually within 1 week.
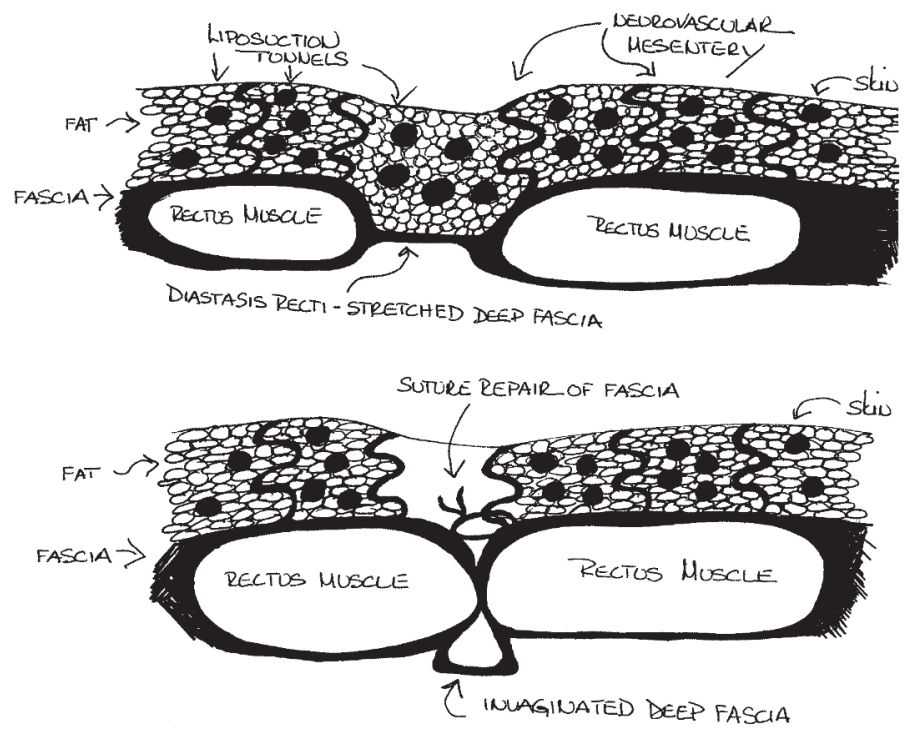

FIG. 3. Rectus sheath plication. Coronal section (above) before and (below) after rectus sheath repair, showing preservation of the most medial neurovascular mesenteries.

\section{Results}

For the 43 patients who underwent liposuction abdominoplasties beginning in 1997 (group III), abdominal incisions were closed with a fluid-tight, absorbable, running subcuticular suture. However, after one minor postoperative wound seroma (in 1999), an interrupted staple closure was adopted in all cases. After their operations, two patients had minor revisions to their neoumbilicus, two others underwent additional (preplanned, staged) liposuction procedures, and one underwent a mastopexy. Most importantly, there were no occurrences of deep venous thrombosis, flap necrosis, sensory loss, or other complications.

Before the operation, the intraabdominal fat content (volume) was evaluated in all 60 patients (groups I, II, and III). The volume was then classified as small, medium, or large. (The author routinely uses this classification when deciding the appropriateness of a rectus sheath plication for a given patient.) In every case, the postoperative appearance of the abdomen correlated directly with the preoperative evaluation of the intraabdominal fat content, regardless of 
whether a diastasis recti repair was performed (23 of 60 patients). More specifically, 25 patients whose intraabdominal fat content was judged as small recovered with a flat abdomen. Eighteen patients estimated to have medium fat volume healed with a medium protrusion, and 17 patients assessed with a large volume ended up with a larger protrusion.

Data were also available for 11 of the 23 patients (groups I, II, and III) in whom diastasis repair was performed. At an average follow-up time of 29 months from the time of diastasis repair, seven patients experienced recurrence of their diastasis when the intraabdominal fat volume was evaluated as large (three patients) or medium (four patients). Four diastasis repair patients whose intraabdominal fat was evaluated as small did not experience a recurrence.

\section{Discussion}

Combining abdominoplasty with liposuction has been discussed extensively in the literature. $4,6,17,29-33$ It is the general consensus that caution be exercised with this surgical combination because of the increased risk for flap necrosis and seroma formation. However, it was the author's need to perform low-risk abdominoplasty on older and/or obese patients that served as a major impetus for the development of his liposuction abdominoplasty technique. Twelve of the 43 patients in group III (28 percent) were 50 years of age or older, and 13 patients (30 percent) were obese (six had a body mass index $>30$, and seven had a body mass index $>35$ ).

Recently, Avelar ${ }^{34}$ described an abdominoplasty that he has been performing since 1998, which incorporates limited liposuction deep to Scarpa's fascia and excision of a strip of suprapubic (and sometimes submammary) skin, without umbilical transposition. On the basis of Avelar's work, Saldanha et al. ${ }^{35}$ reported their experience with an abdominoplasty they have been performing since 2000 , in which more extensive liposuction is combined with an umbilical transposition.

The clinical material presented in this article predates Avelar's work and differs significantly from it. Avelar performs limited liposuction deep to Scarpa's fascia. He tightens the infraumbilical skin by excising a strip of suprapubic skin and then tightens the supraumbilical skin by sliding the skin up and excising a submammary strip of skin. The result is two scars, submammary and pubic, without umbilical transposition. Thus, his abdominal flaps move or slide to a very limited extent. In contrast, the author performs a more complete, full-thickness liposuction, deep and superficial to Scarpa's fascia. 
FIG. 4

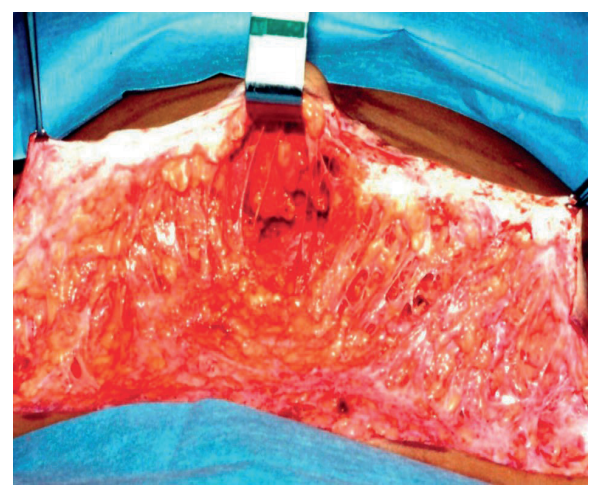

FIG. 5

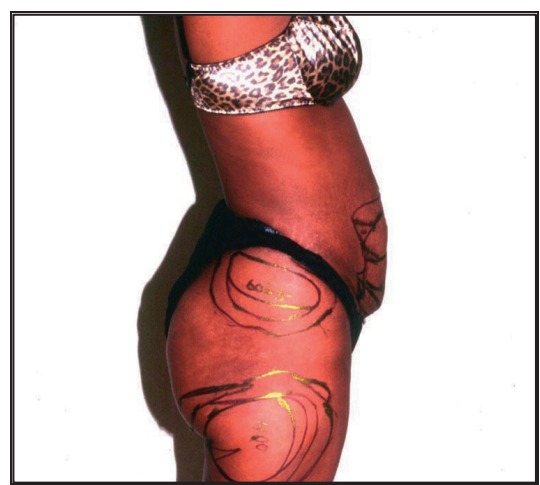

FIG. 4. Another liposuction sliding flap. Note preservation of the most medial perforators. Diastasis repair can be performed, and the perforators can be invaginated and preserved

FIG. 5. A32-year-old woman with a protrudingabdomen, a small amount of intraabdominal fat, and a postural increase inlumbar lordosis

He tightens the supraumbilical and infraumbilical skin by sliding both down to the pubic crease, thereby performing an umbilical transposition and producing only one scar. If desired, the entire infraumbilical skin can be removed. Thus, the flap can be moved to no less a degree than when a traditional abdominoplasty is performed (Fig. 1, above).

The author's procedure also differs from that proposed by Saldanha et al..$^{35}$ It preserves more of the median perforators than the latter technique, even when a rectus sheath repair is performed (Fig. 2, and Fig. 3, above). Vital to the author's technique is his observation that unnamed cutaneous ligaments are present along the midline and surrounding the umbilicus. These freestanding fibrous ligaments, which are separate from the neurovascular perforators, tether the abdominal skin to the aponeuroses of the external oblique and linea alba and are most dense in the periumbilical area. To permit the supraumbilical skin to slide down toward the pubic area (Fig. 2, above), these ligaments must be divided through the umbilical opening and the lower abdominal incision. In contrast to the method of Saldanha et al., ${ }^{35}$ it is not necessary to completely remove the fat above the umbilicus or undermine a 4-cm strip of "subdermal tissue" to gain skin mobility or perform a rectus plication. Rather, the tethering cutaneous ligaments can be divided while most of the important periumbilical and medial perforating blood vessels and nerves are preserved (Fig. 4, above left). Furthermore, during rectus plication, more perforators can be preserved by invaginating them the same way in which the umbilical stump is invaginated (Fig. 3, above). Also, because excision or liposuction of a large panniculus is difficult and time consuming, large panniculi are not removed by full-thickness liposuction or "complementary (open) lipoplasty," as advocated by Avelar ${ }^{34}$ and Saldanha et al. ${ }^{35}$ Rather, the author only performs deep liposuction of the panniculus. This method separates the panniculus from the 
abdominal wall and enables a rapid and bloodless removal, leaving behind areolar tissue and a thin layer of residual fat (Fig. 1, above).

\section{The Sliding Flap}

Liposuction creates sliding flaps by dissecting free their fibrous/neurovascular mesenteries (Fig. $\underline{2}$, above). Flap mobility is obtained by fat removal, the transection of the cutaneous ligaments, and the stretching of the neurovascular mesenteries (Fig. 1, above). The concept of a sliding flap, tethered by a subcutaneous pedicle, is not new; these flaps are used extensively in reconstructive surgery. ${ }^{36}$ Liposuction sliding flaps possess a rich blood supply, evident by the rarity of skin necrosis in the procedure; however, they are not safer than undermined traditional flaps. Clinical judgment should be exercised regarding the extent of liposuction, the thickness of the flap, and the degree of tension. Sliding flaps possess several advantages: they eliminate dead space, can be contoured, and are sensate. The 43 patients who underwent liposuction abdominoplasty (group III) maintained normal skin sensibility because the tissue was not undermined. By comparison, the 19 patients who had traditional abdominoplasties (groups I and II) sustained lower abdominal numbness consistent with disruption of the anterior rami of the intercostal nerves.

\section{Intraabdominal Volume and Diastasis Recti Repair}

Anterior abdominal wall-tightening procedures such as rectus plication are performed to achieve a flat, non-protuberant abdominal appearance. The intraabdominal contents consist of intraabdominal fat and organs; the fat includes both visceral fat and a substantial amount of retroperitoneal fat. ${ }^{37,38}$ Normally, there are marked variations in the volume and position of the abdominal contents. Visceral position is affected by posture, respiratory excursions, and body build. Therefore, a postural increase in lumbar lordosis predisposes to a protruding abdomen even in a thin person (Fig. 5 above right). With the exception of the anterior abdominal wall and the diaphragm/rib cage, most of the abdominal cavity has rigid and nonyielding boundaries. The superior boundary of the abdominal cavity is the diaphragm/rib cage; the inferior boundary is the pelvis. Lateral boundaries are the fleshy parts of obliqui, transverses, and the ilium and iliacus. The anterior boundary is the recti and aponeuroses of obliqui and transversi abdominis, and the posterior boundary is the lumbar vertebrae, crura of the diaphragm, psoas, and quadrati lumborum. ${ }^{37}$ Thus, only the anterior abdominal wall and diaphragm/rib cage-and to a much lesser extent the waist between L-3 and L-5-are pliable and can accommodate an increase in the intraabdominal volume. ${ }^{38-40}$ Furthermore, the abdominal cavity encloses a finite intraabdominal volume. Tightening of the pliable anterior wall pushes the intraabdominal contents (volume) against the only other pliable boundary, the diaphragm. 

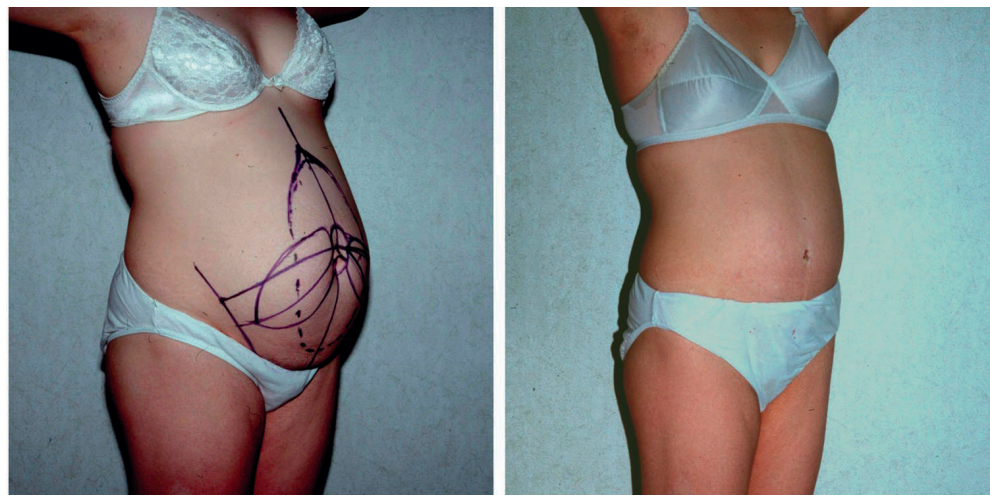

FIG. 6. A 39-year-old, pear-shaped woman with diastasis recti and an umbilical hernia, shown (left) before and (right) 6 months aftera traditionalabdominoplasty. At 6 months, the subcutaneous fat is negligible. The epigastric bulging (not present during the early postoperative period) is solely due to her large intraabdominal volume.

Therefore, the following hypothesis is drawn from the author's clinical experience and this study. Rectus plication (i.e., tightening of the anterior abdominal wall) can only be successful if it is not performed at the expense of diaphragmatic excursion. Consequently, in the presence of a large intraabdominal volume, it cannot be expected that such a tightening procedure will endure. Over time, forceful diaphragmatic/rib cage pressure, coupled with gravity, will restretch the anterior abdominal wall and cause epigastric bulging and recurrence of diastasis (Fig. 6 above). This course of events is exemplified by a high rate of recurrence of abdominal wall hernias in patients with chronic obstructive pulmonary disease.

Consequently, the fat volume of the abdominal contents seems to determine the success or failure of rectus plication. In this preliminary study, the data (although limited by size) suggest that diastasis recti will recur if the intraabdominal fat volume is large enough (seven patients; three patients had large fat volume and four patients had medium volume) and will endure if the intraabdominal fat volume is small (four patients), as reported in the Results section.

Overall, the long-term integrity of diastasis repair has not yet been established. The integrity of rectus sheath diastasis repair at 6 months was discussed by Nahas et al. ${ }^{41}$ and Birdsell et al. ${ }^{42}$ Among the 14 patients reported by Nahas et al., ${ }^{41}$ four patients increased their waist size and one patient remained the same. Birdsell et al..$^{42}$ did not refer to their patients' waist size. AlQattan ${ }^{43}$ reported partial recurrence of abdominal laxity in all of his patients. Additionally, at a mean follow-up time of 64 months, van Uchelen et al. ${ }^{11}$ found that 66 percent of their patients had a reseparation of the recti muscles and 40 percent of cases demonstrated noticeable epigastric bulging that was not present before the operation. Although the effect of visceral fat on abdominoplasty has been discussed by Baroudi ${ }^{6}$ and Matarasso, ${ }^{32}$ they do not question the integrity of long-term diastasis repair, and Matarasso assumes that only the lateral waistline is 
affected. Moreover, neither author views visceral fat as only one component of the total intraabdominal fat volume.

Therefore, the mere presence of diastasis recti should not be an indication for correction, ${ }^{7-9}$ and repair should not be performed under the following conditions: (1) when there is a significant intraabdominal fat volume (Figs. 6 above and 7 below), unless the abdominal wall is exceptionally lax (Fig. 6, above); (2) when a patient might want to become pregnant or does not wish to have the procedure, especially if liposuction and excess skin excision can accomplish a significant improvement (Figs. 8 and 9 below); and (3) when treating high-risk, elderly, and obese patients (Fig. 7 below), because this type of correction increases intraabdominal pressure, which predisposes them to respiratory complications., ${ }^{1,2,7}$ Ultimately, the final result of the abdominoplasty procedure with or without diastasis repair will be both determined and limited by the finite intraabdominal volume contained within the tightened abdomen. This is demonstrated in this study by the direct correlation of the postoperative abdominal appearance and the intraabdominal fat volume in all 23 patients (groups I, II, and III) who underwent diastasis repair and by the direct correlation of the postoperative appearance and the intraabdominal fat volume in all 60 patients.
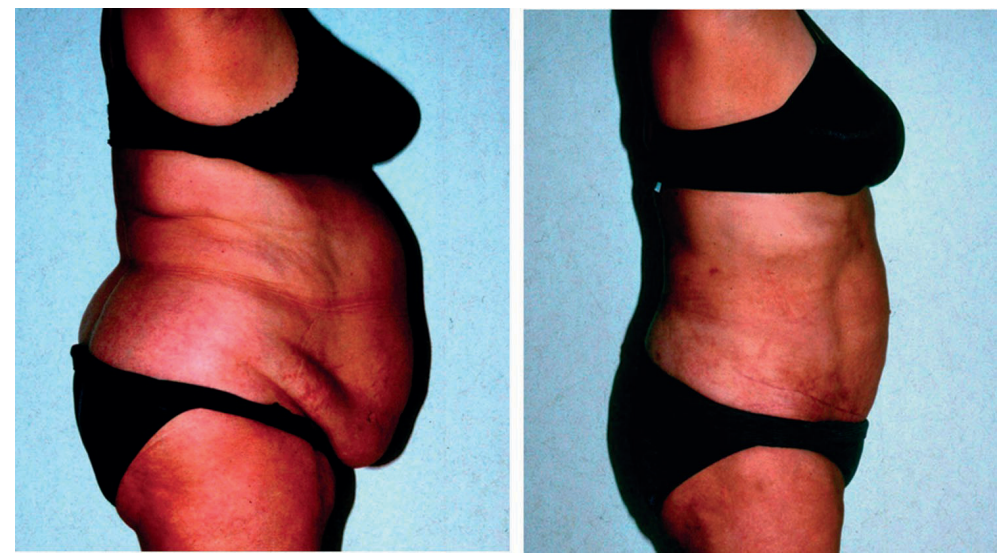

FIG. 7. A 60-year-old obese woman (left) before and (right) 3 months after liposuction abdominoplasty without diastasis repair, followed by liposuction of the trunk and thighs. At 3 months, the subcutaneous fat is negligible. The abdominal protrusion is caused solely by her large intraabdominal volume.

\section{New Evaluation Concepts and a Proposed Classification}

Clinical evaluation of intraabdominal volume is conducted in the standing patient. The muscular abdominal wall is palpated, and the extent of its protrusion is assessed. The volume within can then be quantified. Similarly, the thickness of softer subcutaneous fat can be evaluated, and by gripping as much of the subcutaneous fat as possible, one can tell what the abdomen would look 
like if only the excess fat and skin were to be removed (Fig. 10 below). The degree of the muscular wall's protrusion generally indicates the volume of the intraabdominal fat. The patient's body build also indicates the presence or absence of a large intraabdominal fat content. For example, the dominant presence of thigh fat usually indicates small intraabdominal volume. Conversely, pear-shaped women and many men have large intraabdominal volumes. In addition, the presence of epigastric fullness usually indicates a large volume, whereas an isolated lower abdominal protrusion usually correlates with a lax muscular wall rather than a large intraabdominal fat volume. Asking patients to hold in their abdomen and then breathe out produces epigastric bulging (similar to a postoperative bulge) when a large intraabdominal volume is present (Fig. 6 , above).
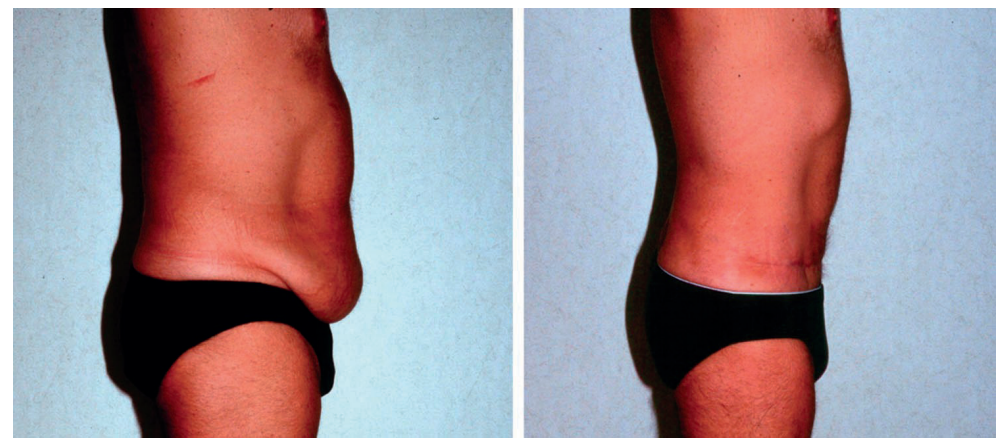

FIG. 8. A 52-year-old man with a large panniculus, diastasis recti, and a small amount of intraabdominal fat. (Left) Before and (right) 10 months after liposcution abdominoplasty without diastasis repair. His result is equivalent to or better than that achieved with tradional abdominoplasty.

Classifications of abdominoplasty, without consideration of the intraabdominal volume, have been described. ${ }^{44}$ Since the abdomen cannot be viewed in isolation, surgical decisions are influenced by a patient's age, weight, health status, smoking history, and physical appearance. For example, a flat abdomen with a tiny waist may contrast with a person's broad shoulders and large muscular thighs (ig. 9). Moreover, the patient's wishes, expectations, and lifestyle should be taken into account. Therefore, an abdominoplasty classification system should influence, but not determine, the surgical treatment. A simple classification of the intraabdominal fat is proposed below.

\section{Intraabdominal Volume: Small, Medium or Large}

A small volume is defined in the standing patient (after discounting the external abdominal fat) as a flat upper muscular abdominal wall and a lower abdominal wall that is either flat or 
somewhat bulging (Figs. 5, 8, and 11). A medium volume is described as a moderately bulging upper and/or lower muscular wall (Figs. 9 and 10). A large volume is characterized by a significantly bulging upper and lower muscular wall (Figs. 6 and 7). Nonetheless, the abdominal fat content is a continuum and may also be small to medium or medium to large, and so on.
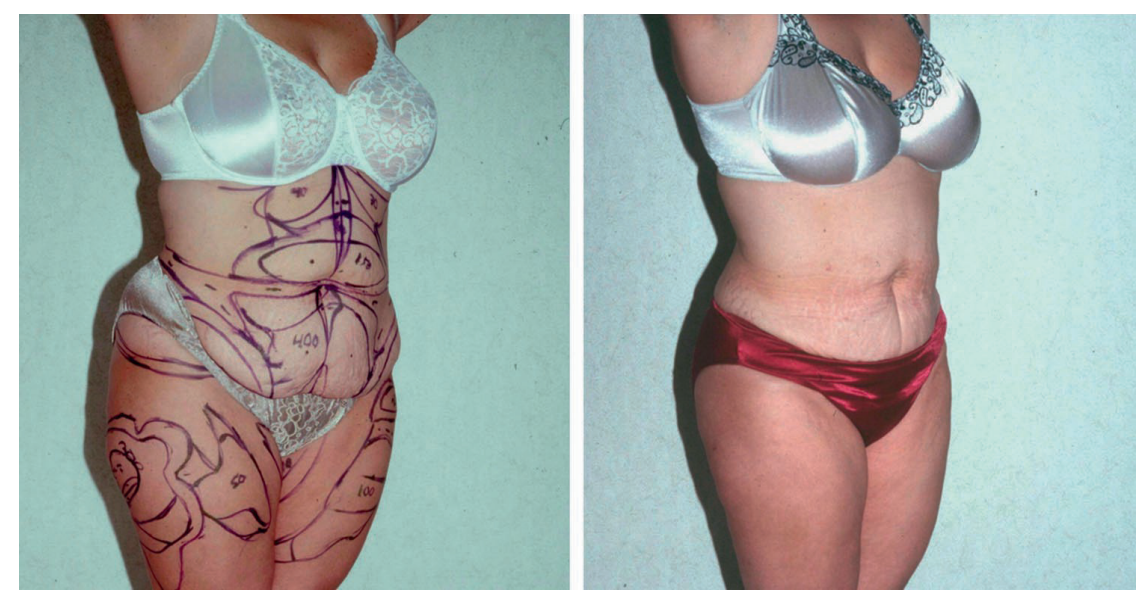

FIG. 9. A 39-year-old woman described herself as having a "full figure" and wished to remain so. She had a hanging panniculus, diastasis recti and a medium amount of intraabdominal fat. (Left) Before and (Right) 4 months after liposuction abdominoplasty without diastasis repair. A smaller waistline would have been disproportional with her muscular thighs and broad upper body.

The presence of diastasis indicates that the anterior abdominal wall has been stretched. As a natural progression of this work, the author believes that the decision to tighten the abdominal wall depends on the intraabdominal fat volume and the muscular wall's laxity (or tightness). The lax (or overstretched) abdominal wall may be viewed as an envelope that is larger than its contents and capable of accommodating more volume without additional stretching. Evaluation of the laxity or tightness of the muscular wall should be performed when the patient is in the supine and upright positions. A lax abdomen is soft and easily compressible laterally and anteroposteriorly. A tight abdomen, by contrast, feels hard and tense especially in the upright patient. An intraoperative assessment (after liposuction of subcutaneous tissue and without the use of a muscle relaxant) can also determine whether a muscular wall is lax enough to allow a low-tension rectus plication.

Therefore, rectus sheath plication is indicated when the anterior abdominal wall is judged to be lax. The degree of correction that can be achieved is both determined and limited by the intraabdominal fat volume (Fig. 6). Ultimately, most patients in this study did not require rectus plication, since muscular wall tightness usually accompanied medium and large intraabdominal fat volumes. In addition, in most cases of small intraabdominal fat volumes, the minimal degree 
of laxity present did not significantly contribute to the abdominal protrusion when evaluated in the standing patient.
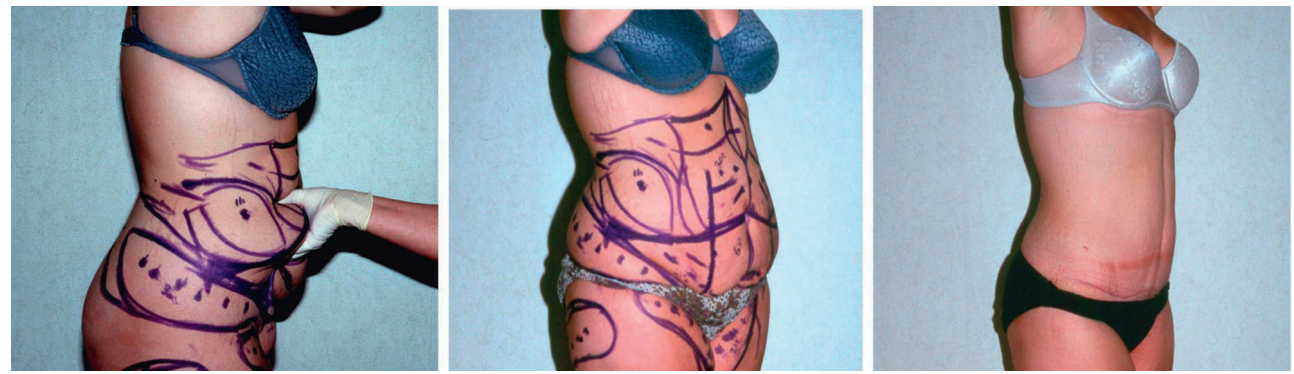

FIG. 10. A 17-year-old girl after massive weight loss, with a hanging panniculus, diastasis recti, and a medium intraabdominal volume. (Left) Note the minimal contribution of the diastasis recti to the abdominal protrusion. (Center) Before and (Right) 2 months after liposuction abdominoplasty without diastasis repair.

More specifically, of the 43 patients in group III, 16 patients were judged to have a small amount of intraabdominal fat and diastasis recti. The muscular wall protruded significantly in two of these patients who underwent diastasis repair with good results.
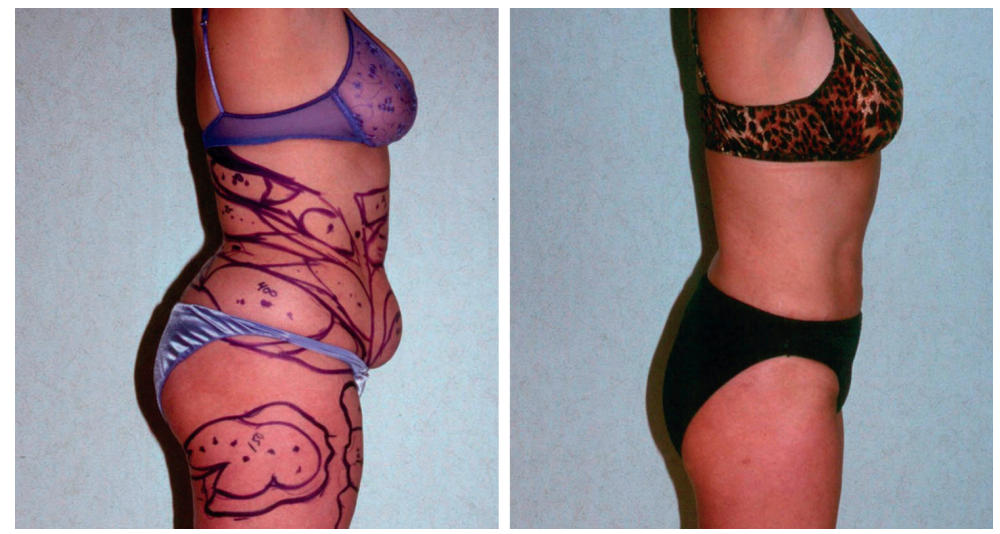

FIG. 11. A 35-year-old woman with a panniculus and a small intraabdominal fat volume. (Left) Before and (Right) 11 months after liposuction abdominoplasty without diastasis repair.

In the remaining 14 patients, the diastasis did not seem to contribute significantly to their abdominal protrusion and they, too, ended up with a flat abdomen without diastasis repair (Fig. 8).

Consequently, diastasis repair is indicated in the presence of a small intraabdominal fat volume only when the muscular wall is significantly protruding in the standing patient (after discounting 
the subcutaneous fat). When the intraabdominal volume is medium (Figs. 9 and 10) or large (Fig. 7), diastasis repair is usually not indicated unless significant muscular laxity is present (ig. 6).

\section{Summary}

This presentation has explored several concepts related to patient selection for abdominoplasty. The abdominal cavity encloses a finite volume of contents within its boundaries, which are rigid and nonyielding, except for the anterior abdominal wall and the diaphragm/rib cage.

Therefore, barring technical problems, the long-term durability of diastasis repair seems to depend on the volume of the abdominal contents. Moreover, diastasis repair appears to be beneficial only when the intraabdominal fat volume is small and the lax muscular abdominal wall protrudes significantly. When the intraabdominal fat volume is medium or large, diastasis repair is not beneficial unless significant abdominal laxity is present. A sensate sliding flap, ${ }^{18,32,36}$ which is created by liposuction and based on multiple neurovascular mesenteries, has several advantages over flaps that are raised by undermining. It can be aggressively defatted, it minimizes dead space, and it has a normal nerve supply. Most importantly, patient satisfaction with this procedure is so high that liposuction abdominoplasty has replaced traditional abdominoplasty in the author's practice.

\section{References}

1. Daane, S. P., and Rockwell, W. B. Analysis of methods for reporting severe and mortal lipoplasty complications. Aesthetic Plast. Surg. 23: 303, 1999.

2. Reinisch, J. F., Bresnick, S. D., Walker, J. W. T., and Rosso, R. F. Deep venous thrombosis and pulmonary embolus after face lift: A study of incidence and prophylaxis. Plast. Reconstr. Surg. 107: 1570, 2001.

3. Stuzin, J. M., Baker, T. J., and Baker, T. M. Deep venous thrombosis and pulmonary embolus after face lift: A study of incidence and prophylaxis (Discussion). Plast. Reconstr. Surg. 107: 1576, 2001.

4. Dillerud, E. Abdominoplasty combined with suction lipoplasty: A study of complications, revisions, and risk factors in 487 cases. Ann. Plast. Surg. 25: 333, 1990.

5. Abramson, D. L. Tumescent abdominoplasty: An ambulatory office procedure. Aesthetic Plast. Surg. 22: 404, 1998.

6. Baroudi, R. Liposuction as an adjunct to a full abdominoplasty revisited (Discussion). Plast. Reconstr. Surg. 106: 1203, 2000.

7. Pitanguy, V. Abdominal lipectomy: An approach to it through an analysis of 300 consecutive cases. Plast. Reconstr. Surg. 40: 384, 1967.

8. Regnault, P. Abdominoplasty by the W technique. Plast. Reconstr. Surg. 55: 265, 1975.

9. Regnault, P. Abdominal dermolipectomies. Clin. Plast. Surg. 2: 411, 1975. 
10. Zecha, P. J., and Missotten, F. E. Pseudocyst formation after abdominoplasty: Extravasations of MoretLavallée. Br. J. Plast. Surg. 52: 500, 1999.

11. van Uchelen, J. H., Kon, M., and Werker, P. M. N. The long-term durability of plication of the anterior rectus sheath assessed by ultrasonography. Plast. Reconstr. Surg. 107: 1578, 2001.

12. Wilkinson, T. S., and Swartz, B. E. Individual modifications in body contour surgery: The limited abdominoplasty. Plast. Reconstr. Surg. 77: 779, 1986.

13. Wilkinson, T. S. Limited abdominoplasty techniques applied to complete abdominal repair. Aesthetic Plast. Surg. 18: 49, 1994.

14. Greminger, R. F. The mini-abdominoplasty. Plast. Reconstr. Surg. 79: 356, 1987.

15. Eaves, F. F., III, Nahai, F., and Bostwick, J., III. Endoscopic abdominoplasty and endoscopically assisted miniabdominoplasty. Clin. Plast. Surg. 23: 599, 1996.

16. Zukowski, M. L., Ash, K., Spencer, D., Malanoski, M., and Moore, G. Endoscopic intracorporal abdominoplasty: A review of 85 cases. Plast. Reconstr. Surg. 102: 516, 1998.

17. Shestak, K. C. Liposuction as an adjunct to a full abdominoplasty revisited (Discussion). Plast. Reconstr. Surg. 106: 1205, 2000.

18. Lockwood, T. High-lateral-tension abdominoplasty with superficial fascial system suspension. Plast. Reconstr. Surg. 96: 603, 1995.

19. Lockwood, T. E. Lower-body lift. Aesthetic Surg. J. 21: 355, 2001.

20. Brauman, D. Lipoplasty: A case for a low-volume procedure. Aesthetic Surg. J. 20: 373, 2000.

21. Burk, R. W., III, Guzman-Stein, G., and Vasconez, L. O. Lidocaine and epinephrine levels in tumescent technique liposuction. Plast. Reconstr. Surg. 97: 1379, 1996.

22. Fodor, P. B. Lipoplasty: Another plea for safety! Aesthetic Plast. Surg. 22: 399, 1998.

23. Klein, J. A. Tumescent technique for regional anesthesia permits lidocaine doses of $35 \mathrm{mg} / \mathrm{kg}$ for liposuction. J. Dermatol. Surg. Oncol. 16: 248, 1990.

24. Klein, J. A. Tumescent technique for local anesthesia improves safety in large-volume liposuction. Plast. Reconstr. Surg. 92: 1085, 1993.

25. Ostad, A., Kageyama, N., and Moy, R. L. Tumescent anesthesia with a lidocaine dose of $55 \mathrm{mg} / \mathrm{kg}$ is safe for liposuction. Dermatol. Surg. 22: 921, 1996.

26. Alegria-Perén, P., Barba-Gómez, J., and Guerrero-Santos, J. Total corporal contouring with megaliposuction (120 consecutive cases). Aesthetic Plast. Surg. 23: 93, 1999.

27. Physician's Desk Reference, 54th Ed. Montvale, N.J.: Medical Economics, 2000. Pp. 636-638.

28. Rohrich, R. J., Beran, S. J., and Fodor, P. B. The role of subcutaneous infiltration in suction-assisted lipoplasty: A review. Plast. Reconstr. Surg. 99: 514, 1997.

29. Cardenas-Camarena, L., and Gonzalez, L. E. Large-volume liposuction and extensive abdominoplasty: A feasible alternative for improving body shape. Plast. Reconstr. Surg. 102: 1698, 1998.

30. Heppe, H. P. Combined liposuction with abdominoplasty (Letter). Plast. Reconstr. Surg. 108: 577, 2001.

31. Matarasso, A. Liposuction as an adjunct to a full abdominoplasty. Plast. Reconstr. Surg. 95: 829, 1995. 
32. Matarasso, A. Liposuction as an adjunct to a full abdominoplasty revisited. Plast. Reconstr. Surg. 106: 1197, 2000 .

33. Nguyen, T. T., Kim, K. A., and Young, R. B. Tumescent mini abdominoplasty. Ann. Plast. Surg. 38: $209,1997$. 34. Avelar, J. M. Abdominoplasty without panniculus undermining and resection: Analysis and 3-year followup of 97 consecutive cases. Aesthetic Surg. J. 22: 16, 2002.

35. Saldanha, O. R., de Souza Pinto, E. B., Matos, W. N., Jr., Lucon, R. L., Magalhães, F., and Bello, É. M. L. Lipoabdominoplasty without undermining. Aesthetic Surg. J. 21: 518, 2001.

36. Jackson, T. I. Local Flaps in Head and Neck Reconstruction. St. Louis: C.V. Mosby, 1985. Pp. 12-15, 240.

37. Jamieson, E. B. A Companion to Manuals of Anatomy, 7th Ed. London: Oxford University Press, 1965. Pp. $361,628$.

38. Grant's Atlas of Anatomy, 6th Ed. Baltimore: Williams \& Wilkins, 1972. Pp. 24, 104, 2-110, 190.297.

39. Warwick, R., and Williams, P. L. (Eds.). Gray's Anatomy, 35th British Ed. Philadelphia: W.B. Saunders, 1973. Pp. 1254-1256, 519-527.

40. Adams, J. T. Abdominal wall, omentum, mesentery, and retroperitoneum. In S. I. Schwartz (Ed.), Principles of Surgery, 3rd Ed. New York: McGraw-Hill, 1974. Pp. 1451-1457.

41. Nahas, F. X., Augusto, S. M., and Ghelfond, C. Should diastasis recti be corrected? Aesthetic Plast. Surg. 21: 285, 1997.

42. Birdsell, D. C., Gavelin, G. E., Kemsley, G. M., and Hein, K. S. "Staying power": Absorbable vs. nonabsorbable. Plast. Reconstr. Surg. 68: 742, 1981.

43. Al-Qattan, M. M. Abdominoplasty in multiparous women with severe musculoaponeurotic laxity. Br. J. Plast. Surg. 50: 450, 1997.

44. Matarasso, A. Abdominoplasty: A system of classification and treatment for combined abdominoplasty and suction-assisted lipectomy. Aesthetic Plast. Surg. 15: 111, 1991. 
Chapter 4.

Diastasis Recti: Clinical Anatomy

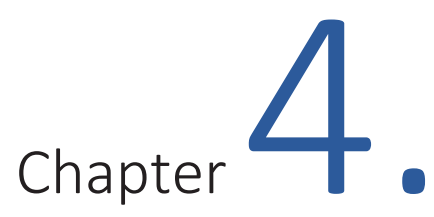

\section{Diastasis Recti: Clinical Anatomy}

PLASTIC AND RECONSTRUCTIVE SURGERY VOLUME 122

Issue 5 - p 1564-1569

November 2008 



\title{
Chapter 4. \\ Diastasis Recti: Clinical Anatomy
}

Plastic and Reconstructive Surgery: November 2008 - Volume 122 - Issue 5 - p 1564-1569

\author{
Daniel Brauman, MBBS, MD
}

White Plains, N.Y

From the Department of Plastic Surgery, Cornell University, Weill Medical College. Received for publication February 22, 2008; accepted April 28, 2008.

Background: Enduring diastasis repair is one of the yardsticks by which a successful abdominoplasty is measured, because the presence and size of diastasis recti are thought to be reliable indicators of abdominal wall laxity and protrusion. The author's study of the "normal" anatomy of diastasis challenges these concepts about diastasis recti.

Methods: Ninety-two consecutive abdominoplasty patients, in whom intraoperative measurements of the linea alba were taken, were included in this study. The degree of abdominal wall protrusion was quantified by estimating the intra-abdominal fat volume in the upright patient as large, medium, or small.

Results: The most striking anatomical finding was that the linea alba has a limited range of stretch, most commonly between 1 and 2 inches, regardless of the extent of the abdominal girth. Moreover, eight patients with diastasis did not manifest abdominal protrusion, and in five patients, diastasis was absent, although a significant protrusion was present. Also, the site of the widest diastasis (supraumbilical/infraumbilical) frequently did not correspond to the site of the protrusion.

Conclusions: Contrary to current thought, abdominal wall protrusions are caused by the stretching of the entire abdominal wall and not only the linea alba. Thus, significant abdominal wall protrusions may occur without diastasis and flat abdomens may exhibit a diastasis. Abdominal protrusion should replace diastasis as the prime indicator of abdominal wall laxity; stretching and the decision to perform diastasis repair should be influenced primarily by the evaluation of the protrusion rather than the diastasis.

Diastasis repair by plication of the rectus sheath is an integral part of most abdominoplasty procedures. As such, understanding the mechanism and natural history of abdominal wall stretching and protrusion is of paramount importance. The literature, however, is lacking in clinical anatomical studies of diastasis. Rath et al. ${ }^{1}$ defined the size of the normal linea alba in 40 fresh cadavers. 
Van Uchelen et al. $^{2}$ studied the durability of diastasis repair postoperatively by using ultrasonography. Birdsell et al. ${ }^{3}$ studied the durability of diastasis repair by placing metal vascular clips at the site of repair and obtaining radiographs postoperatively, and Nahas et al. ${ }^{4}$ studied the durability of diastasis repair using computed tomographic scanning postoperatively. Except for Rath et al., the above authors studied the durability of midline plication, which they equated with a successful result. However, they did not allow for a baseline description of the "normal" anatomy of diastasis recti and for the understanding that the object of a successful abdominoplasty is a flat abdomen.

\section{Patients and Methods}

Ninety-two consecutive abdominoplasty patients (2004 to 2007) with documented intraoperative measurements of diastasis were included in this study. There were 91 women and one man; their data are summarized in Table 1.

\section{Table 1. Patient Data}

\begin{tabular}{lccc}
\hline & Age $(\mathbf{y r})$ & Weight $(\mathbf{l b})$ & Body Mass Index \\
\hline Range & $21-72$ & $103-307$ & $20.8-41.7$ \\
Mean & 45 & 166.7 & 29.8 \\
\hline
\end{tabular}

Patients were divided into three groups based on their intra-abdominal fat volume/content, which was estimated by assessing the extent of abdominal wall protrusion (in the upright position). The fat volume was classified as small, medium, or large (Figs. 1 through 3 ). The width of the diastasis was measured intraoperatively at its widest location, usually at or within 1 inch of the umbilicus, and measurements were rounded up to the nearest 0.5 inch because of respiratory movement. Measurements greater than 0.5 inches were considered to indicate diastasis. ${ }^{5}$ Outpatient surgery was performed in an accredited office surgical suite under local anesthesia with sedation using the liposuction abdominoplasty technique described previously by the author. ${ }^{6,7}$

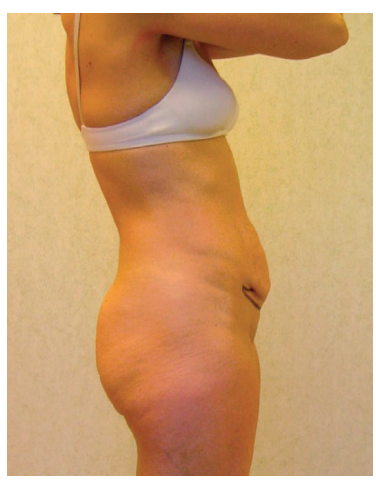

Fig.1. Smallintra-abdominalfatvolume.

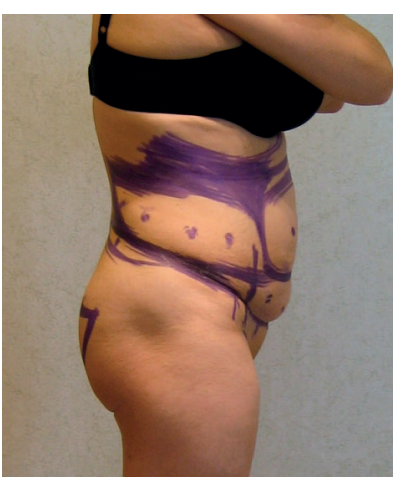

Fig.2. Mediumintra-abdominalfatvolume.

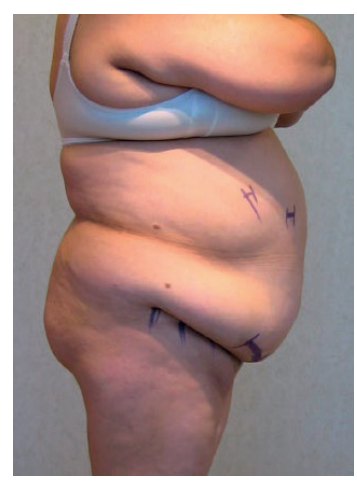

Fig.3. Largeintra-abdominalfatvolume. 


\section{Results}

There were no major complications or problems, such as death, deep venous thrombosis, major infections, tissue necrosis, unplanned hospital admissions, or transfusions. Table 2 and Figure 4 summarize the sizes of the diastases in each group; although the size of the diastases tended to increase with larger abdominal protrusions, smaller abdominal wall protrusions frequently displayed similarly wide diastases (Figs. 5 through 7). In 83 percent of cases, the width of the diastases was limited to between 1 and 2 inches, indicating that most commonly the stretch of the linea alba is restricted and beyond that range it stops stretching. Moreover, eight patients with diastasis did not manifest significant abdominal protrusion, and in five patients, diastasis was absent, although a sizable protrusion was present. An additional observation was that, frequently, the site of the widest diastasis did not correspond to the site of the protrusion in the upright patient (Figs. 1 and 5).

Table 2. Diastasis Sizes for All Patients*

\begin{tabular}{|c|c|c|c|c|c|c|}
\hline & \multicolumn{6}{|c|}{ Size of Diastasis } \\
\hline & $\begin{array}{c}<0.5 \text { Inch } \\
(\%)\end{array}$ & $\begin{array}{c}1.0 \text { Inch } \\
(\%)\end{array}$ & $\begin{array}{c}1.5 \text { Inches } \\
(\%)\end{array}$ & $\begin{array}{c}2.0 \text { Inches } \\
(\%)\end{array}$ & $\begin{array}{c}2.5 \text { Inches } \\
(\%)\end{array}$ & $3.0 \underset{(\%)}{\text { Inches }}$ \\
\hline Group I, small content (26 patients) & $2(8)$ & $12(46)$ & $9(35)$ & $3(11)$ & 0 & 0 \\
\hline Group II, medium content ( 37 patients) & $4(11)$ & $10(27)$ & $11(30)$ & $6(16)$ & $4(11)$ & $2(5)$ \\
\hline Group III, large content ( 29 patients) & $1(3)$ & $1(3)$ & $12(41)$ & $12(41)$ & $3(10)$ & 0 \\
\hline Total (92 patients) & 7 & 23 & 32 & 21 & 7 & 2 \\
\hline
\end{tabular}

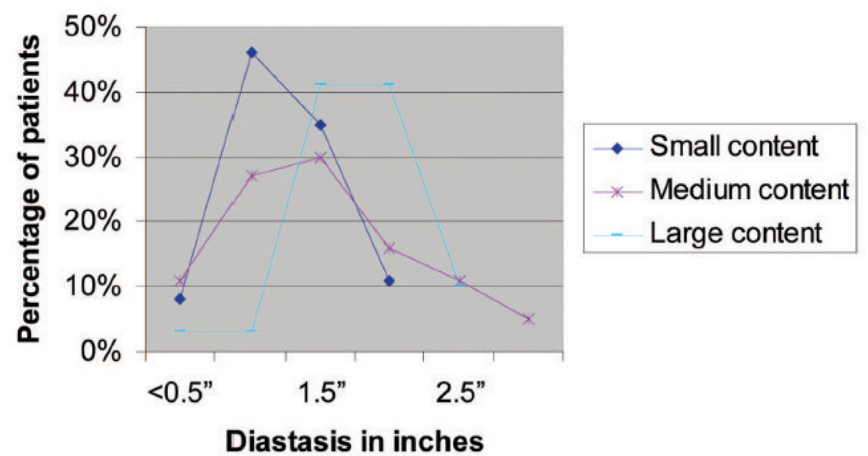

Fig. 4. Graph of width of diastasis plotted against the number (percentage) of patients. Note the lack of a linear relationship. 

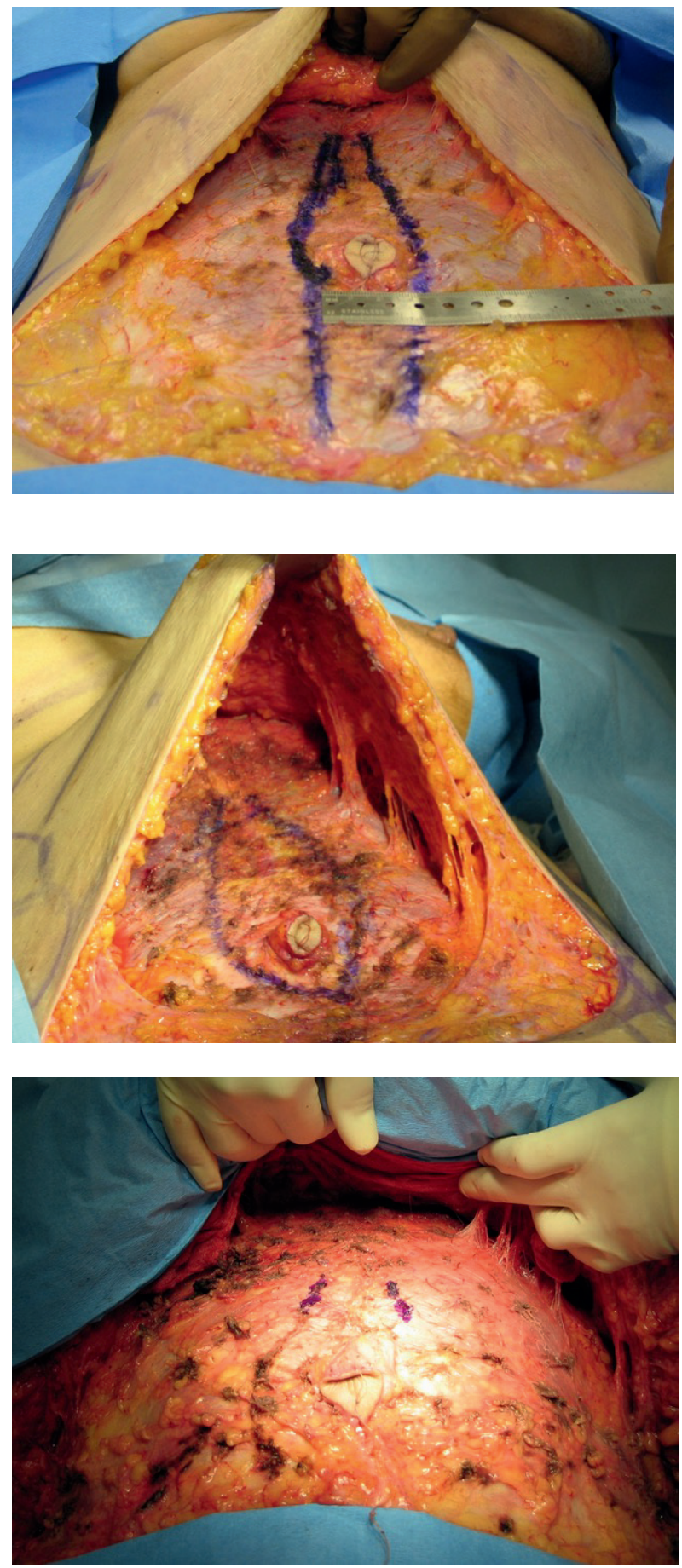

FIG. 5. Intraoperative photograph of the patient presented in Figure 1 shows a small intra-abdominal fat volume. Note the 1-inch infraumbilical diastasis. Also note the much wider supraumbilical diastasis (which appears narrower because of the direction of the photograph). The maximal muscular protrusion was infraumbilical.

FIG. 6. Intraoperative photograph of the patient presented in Figure 2 shows a medium intra-abdominal fat volume. Note the 2-inch diastasis, which is predominantly supraumbilical. The maximal muscular protrusion was central.

FIG. 7. Intraoperative photograph of the patient in Figure 3 shows a large intraabdominal fat volume. Note the stretched out, distended abdominal wall and the relatively narrow diastasis measuring 1.5 inches at the umbilicus. The maximal muscular protrusion was supraumbilical. 
To examine the effect of previous midline abdominal surgery, the 92 patients were subdivided into those who had undergone previous midline abdominal surgery (47 patients) and those who had not ( 45 patients). The results are shown in Tables 3 and 4 . The data show that most of the patients in both subgroups still displayed a limited diastasis measuring between 1 and 2 inches regardless of protrusions/waist size, as did those in the combined group of 92 patients.

Table 3. Data for 47 Patients Who Had Undergone a Previous Midline Abdominal Operation*

\begin{tabular}{|c|c|c|c|c|c|c|}
\hline & \multicolumn{6}{|c|}{ Size of Diastasis } \\
\hline & $\begin{array}{c}<0.5 \text { Inch } \\
(\%)\end{array}$ & $\begin{array}{c}1.0 \text { Inch } \\
(\%)\end{array}$ & $\begin{array}{c}1.5 \text { Inches } \\
(\%)\end{array}$ & $\underset{(\%)}{2.0 \text { Inches }}$ & $\begin{array}{c}2.5 \text { Inches } \\
(\%)\end{array}$ & $\begin{array}{c}3.0 \text { Inches } \\
(\%)\end{array}$ \\
\hline Group I, small content (10 patients) & 0 & $5(50)$ & $4(40)$ & $1(10)$ & 0 & 0 \\
\hline Group II, medium content (20 patients) & $1(5)$ & $5(25)$ & $9(45)$ & $3(15)$ & 0 & $2(10)$ \\
\hline Group III, large content ( 17 patients) & $1(6)$ & $1(6)$ & $7(14)$ & $6(35)$ & $2(12)$ & 0 \\
\hline Total (47 patients) & 2 & 11 & 20 & 10 & 2 & 2 \\
\hline
\end{tabular}

the large content group had diastasis measuring between 1 and 2 inches, indicating that regardless of previous midline surgery, diastais was still limited and of similar dimensions in differing protrusions/waist sizes.

Table 4. Data for 45 Patients Who Had Not Undergone a Previous Midline Abdominal Surgery*

\begin{tabular}{|c|c|c|c|c|c|c|}
\hline & \multicolumn{6}{|c|}{ Size of Diastasis } \\
\hline & $\begin{array}{c}<0.5 \text { Inch } \\
(\%)\end{array}$ & $\underset{(\%)}{1.0 \text { Inch }}$ & $\begin{array}{l}1.5 \text { Inches } \\
(\%)\end{array}$ & $\begin{array}{c}2.0 \text { Inches } \\
(\%)\end{array}$ & $\begin{array}{c}2.5 \text { Inches } \\
(\%)\end{array}$ & 3.0 Inches \\
\hline Group I, small content (16 patients) & $2(12)$ & $7(44)$ & $5(31)$ & $2(12)$ & 0 & 0 \\
\hline Group II, medium content (17 patients) & $3(18)$ & $5(29)$ & $2(12)$ & $3(18)$ & $4(23)$ & 0 \\
\hline Group III, large content (12 patients) & 0 & 0 & $5(42)$ & $6(50)$ & $1(8)$ & 0 \\
\hline Total (45 patients) & 5 & 12 & 12 & 11 & 5 & 0 \\
\hline
\end{tabular}

*Seventy-eight percent ( 35 of 45$)$ had diastasis between 1 and 2 inches; 87 percent of the small content group and 92 percent of the lar
content group measured between 1 and 2 inches, indicating measurements similar to those obtained for those with previous surgery.

A chi-square test was performed on the data in Table 2 combining the last two columns with the smallest cell sizes. The results were as follows: a chi-square statistic of $22.547,8$ degrees of freedom, and a value of $p=0.004$, indicating a significant association. The data in Tables 3 and $\underline{4}$ have total numbers less than 50 and cell sizes less than 5 . Given that the sample size is small, a significant association cannot be established with certainty. However, the data clearly indicate that regardless of the intra-abdominal volume/waist size, the great majority of the measurements exhibited a limited range between 1 and 2 inches.

\section{Discussion}

Quantification of intra-abdominal volume in clinical practice is problematic. The quantification could be readily performed radiologically by computed tomographic, ultrasound, or magnetic resonance imaging analysis. However, this is impractical in a medical office setting. Nevertheless, there is an urgent need for medical doctors and not just plastic surgeons to be able to clinically quantify intra-abdominal fat volume. 


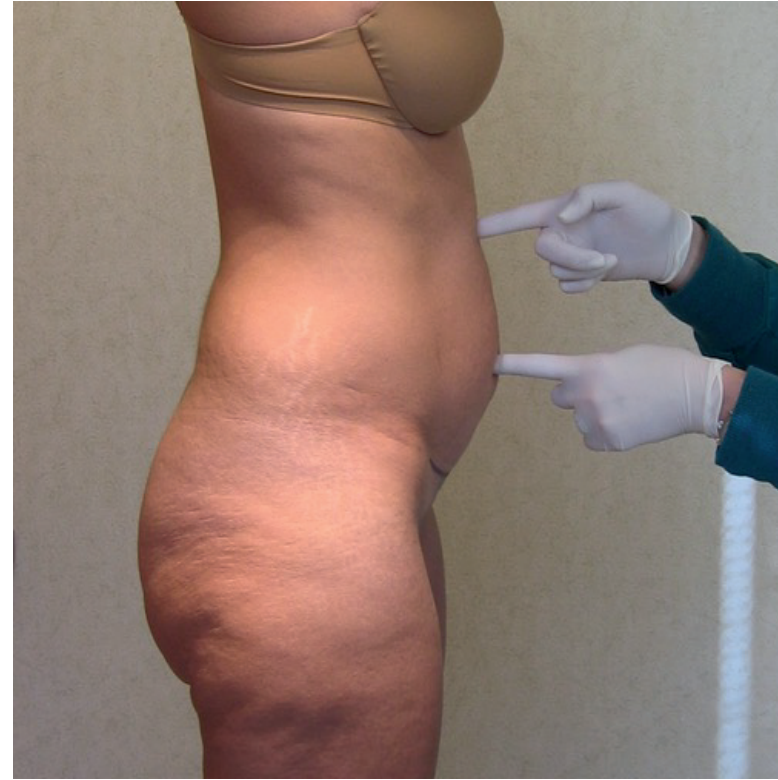

Fig. 8. Maximal muscular protrusion. The examiner's fingers are resting against the muscular abdominal wall in the midline of the supraumbilical and infraumbilical regions. This patient's protrusion is maximal in the infraumbilical area, and she is estimated to have a small intra-abdominal fat volume.
Intra-abdoinal fat is currently thought to be composed of adipose tissue functioning as an endocrine organ and interacting with the endocannabinoid system, causing the metabolic syndrome. ${ }^{8}$ Medical researchers attempted to quantify intra-abdominal fat volume and have proposed measurements such as waist circumference and, more recently, waist-to-hip ratio. ${ }^{9,10}$ The author has not found these measurements to be accurate because they include subcutaneous fat, which is highly variable. In addition, for soft-tissue measurements to be reproducible, they should refer to bony landmarks. Instead, in 2002 the author proposed a simple clinical classification that he has been using in his practice for intra-abdominal fat

quantification: small, medium, and large. ${ }^{6}$ The classification is user-friendly and is based on the midline palpation of the muscular abdominal wall in the standing patient. It is fairly easy to define the two extremes: small and large volumes. Medium volume is somewhere in between. Anatomically, the site of the maximal muscular protrusion in the midline is generally an accurate guide (Fig. 8). Supraumbilical (epigastric) protrusions generally denote a large volume. Infraumbilical protrusions (hypogastric) generally denote a small volume, and umbilical (central) protrusions denote a medium volume. Other factors, such as posture (pelvic tilt and lordosis) and previous midline abdominal surgery, affect the site of the maximal muscular protrusion. For example, an increase in lumbar lordosis and previous lower midline abdominal surgery usually displace the maximal muscular protrusion superiorly, whereas a decrease in lordosis lowers it.

Anatomy textbooks describe the linea alba as 0.5 inches wide superior to the umbilicus and narrower inferior to it. ${ }^{5}$ This study demonstrates that the stretching of the linea alba is usually limited to a fairly narrow range. When this narrow range of 2 inches or even 3 inches is compared with the much wider range of people's waist sizes, it should be concluded that contrary to current theories on the subject, the protrusion and stretching of the anterolateral abdominal wall are caused by the stretching of the entire musculofascial abdominal wall and not only the linea alba (diastasis). In a much larger series of patients, the author has occasionally observed diastases 
wider than 3 inches, but this is the exception rather than the norm. In addition, the presence of diastasis merely indicates past or present abdominal wall stretching, and patients with diastasis and without significant protrusion have been observed.

In any sizable sample of patients, the effect of previous midline abdominal surgery has to be reckoned with, because the purpose of an abdominoplasty is to correct abdominal deformities resulting from both pregnancy/weight gain and previous abdominal operations. Theoretically, previous midline abdominal surgery may have a dual and opposing effect on diastasis. Midline surgery may tighten the linea alba, especially when "large bites" are taken to the edge of the recti muscles. Conversely, it may weaken the linea alba when the midline scar is subsequently subjected to increasing intra-abdominal pressure caused by obesity or pregnancy. Other variables to be considered are the duration after the surgery, the site of the incision, and the individual nature of the healing. For example, if many years have passed since midline surgery has been performed, increasing intra-abdominal pressure is more likely to restretch the "repaired" linea alba. In addition, it has been observed that epigastric repairs have a tendency to restretch after repair more than hypogastric repairs. ${ }^{2,6,11}$ In view of the above, the assumption was that in a large enough sample of patients, the opposing effects of previous midline abdominal surgery would cancel each other out. This assumption proved to be correct. Nevertheless, factoring previous surgery is complex and has to be performed on a case-by-case basis.

Rath et al. ${ }^{1}$ published the only article found that is devoted to the anatomy of the linea alba. Studying 40 fresh cadavers, they observed that the linea alba is usually widest at the umbilicus. In addition, age older than 45 years was found to increase the width of the supraumbilical and infraumbilical diastasis by only $5 \mathrm{~mm}$. The size of the widest diastasis, the umbilical ring diastasis was not affected by age. The observations by Rath et al. are corroborated in this study.

Van Uchelen et al. ${ }^{2}$ examined recurrent diastases after abdominoplasty using ultrasonography. The diastases ranged between 1 and $4.2 \mathrm{~cm}$. In 17 cases, the supraumbilical separation was larger than the infraumbilical and in six the reverse was true. The data reported by Birdsell et al. 3 are mentioned in passing. The diastasis in their study ranged from 4 to $6 \mathrm{~cm}$ and was widest in the hypogastric area. Because their 30 female subjects were described as "nonobese" (with, most likely, scaphoid abdomens), they would be expected to have had wider hypogastric diastases. Nahas et al..measured the diastasis $3 \mathrm{~cm}$ above and $2 \mathrm{~cm}$ below the umbilicus in 14 patients, and their data show a range of 1 to $4.5 \mathrm{~cm}$, with the supraumbilical diastases wider than the infraumbilical diastases. Even though the above authors describe their findings accurately, the point missed is that in view of the findings of this study, a successful abdominoplasty should result in a flat abdomen and not in an enduring diastasis repair.

The results indicate that stretching of the linea alba is limited to 2 inches in the majority of cases ( 82 percent) and that linea alba stretching beyond 2.5 inches occurs only in a small number of cases [two of 92 patients ( 2 percent)]. Therefore, the reliance on linea alba stretching as an indicator of abdominal wall stretching is erroneous. 
Most frequently, the linea alba has a restricted upper limit beyond which it stops stretching. This stretching is not only limited but also similar in dimension in a wide range of individuals with a wide range of waist sizes. Additional abdominal distention is caused by the stretching of the entire anterolateral musculofascial wall. Therefore, the size of the diastasis is not a reliable indication of the extent of abdominal laxity and protrusion. The presence of abdominal laxity and protrusion is not synonymous with the presence of a diastasis of the recti muscles; conversely, flat abdomens may exhibit significant diastases. The site of maximal abdominal protrusion, namely, supraumbilical or infraumbilical, may not correlate with the site of the widest diastasis (Figs. 1 and 5). Therefore, the decision of whether or not to undertake muscle plication should be influenced more by the upright examination of the patient than by the mere presence of a significant diastasis in the supine patient. Moreover, the success of an abdominoplasty should be judged by the flat appearance of the abdomen and not by whether or not the diastasis repair endured.

\section{References}

1. Rath, A. M., Attali, P., Dumas, J. L., Goldlust, D., Zhang, J., and Chevrel, J. P. The abdominal linea alba: An anatomo-radiologic and biochemical study. Surg. Radiol. Anat. 18: 281, 1996.

2. Van Uchelen, J. H., Kon, M., and Werker, P. M. N. The long-term durability of plication of the anterior rectus sheath assessed by ultrasonography. Plast. Reconstr. Surg. 107: 1578, 2001.

3. Birdsell, D. C., Gavelin, G. E., Kemsley, G. M., and Hein, K. S. "Staying power": Absorbable vs. nonabsorbable. Plast. Reconstr. Surg. 68: 742, 1981.

4. Nahas, F. X., Augusto, S. M., and Ghelfond, C. Should diastasis recti be corrected? Aesthetic Plast. Surg. 21: 285, 1997.

5. Jamieson, E. B. A Companion to Manuals of Practical Anatomy, 7th Ed. London: Oxford University Press, 1950. Pp. 361, 368.

6. Brauman, D. Liposuction abdominoplasty: An evolving concept. Plast. Reconstr. Surg. 112: 288, 2003.

7. Brauman, D. Lipoplasty: A case for a low-volume procedure. Aesthetic Plast. Surg. 20: 373, 2000.

8. Kershaw, E. E., and Flier, J. S. Adipose tissue as an endocrine organ. J. Clin. Endocrinol. Metab. 89: 2548, 2004.

9. Yusuf, S., Hawken, S., Ounpuu, S., et al. Obesity and the risk of myocardial infarction in 27,000 participants from 52 countries: A case-control study. Lancet 366: 1640, 2005. 
10. Romero-Corral, A., Montori, V. M., Somers, V. K., et al. Association of bodyweight with total mortality and with cardiovascular events in coronary artery disease: A systematic review of cohort studies. Lancet 368: 666, 2006.

11. American Society of Plastic Surgeons. Practice parameter for abdominoplasty and panniculectomy unrelated to obesity or massive weight loss. Arlington Heights, III.: American Society of Plastic Surgeons. July 20, 2006. 

Chapter 4a Letters. Diastasis Recti: Clinical Anatomy

\title{
LETTERS
}

\author{
Diastasis Recti: \\ Clinical Anatomy
}

Diastasis Recti: Clinical Anatomy

PLASTIC AND RECONSTRUCTIVE SURGERY VOLUME 123

Issue 6 - p 1885-1886

June 2009

Factors that May Influence Failure of the Correction of the Musculoaponeutroic Deformities of the Abdomen PLASTIC AND RECONSTRUCTIVE SURGERY VOLUME 124

Issue 1- p 334-335

July 2009 



\section{Diastasis Recti: Clinical Anatomy}

\section{Sir:}

We read with interest the article by Dr. Brauman regarding diastasis recti and the sources of abdominal wall protrusions. We would like to commend the author on addressing a subject that is frequently underevaluated with respect to abdominal contouring. Abdominal protrusion can be a result of multiple factors including extra-abdominal fat, intraabdominal fat, diastasis recti, abdominal wall hernia, as well as global myofascial laxity.

Traditional abdominoplasty techniques address extra- abdominal fat through liposuction and resection, and correction of diastasis recti and abdominal wall hernia(s) through direct fascial repair including plication of the linea alba. Intraabdominal fat can be a significant source of abdominal protrusion, but it is not addressed in traditional abdominoplasty techniques.

The last potential source of abdominal wall protrusion is global myofascial laxity.

Abdominoplasty patients with significant abdominal protrusion and relatively little intra- and extra-abdominal fat frequently have abdominal wall laxity that is much greater than the degree of diastasis recti present. Deferring plication or plicating only sufficiently to de-crease the width of the linea alba frequently results in inadequate correction of abdominal wall laxity and the persistence of abdominal wall protrusion.

Based on these observations, we prefer to use the term myofascial plication to better describe the process of global myofascial laxity correction. ${ }^{1}$ The technique is similar to standard diastasis recti plication; however, plication involves imbricating a wider portion of the anterior rectus sheath as determined by preoperative fascial plication shortens the width of the anterior rectus sheath and in turn pulls the paired linea semilunaris toward midline, resulting in re-approximation of the medial edges of the rectus muscles. ${ }^{1}$ This entire process results in overall tightening of the abdominal wall as a myofascial unit. The frequency and significance of global myofascial laxity are often under-appreciated in the process of abdominal wall tightening for the abdominoplasty patient.

DOI: 10.1097/PRS.0b013e3181a84920

Remus Repta, M.D.

Advanced Aesthetic Associates Phoenix, Ariz.

Joseph P. Hunstad, M.D.

The Hunstad Center Charlotte, N.C.

Correspondence to Dr. Repta Advanced Aesthetic Associates

9225 N 3rd Street, Suite 103

Phoenix, Ariz. 85020

drrepta@gmail.com

\section{References}

1. Hunstad JP, Repta R. The Atlas of Abdominoplasty. London: Saunders;2008. 


\section{Reply}

Sir:

I appreciate the letter by Drs. Repta and Hunstad. They point out that they have identified a group of patients whose abdominal wall laxity is so marked that merely correcting their diastasis will lead to under-correction of their abdominal protrusion. They refer to this finding as "global myofascial laxity." In doing so, they have perceptively addressed an important aspect of abdominal wall reconstruction and diastasis recti repair: laxity. The authors proceed to describe their method of diastasis repair, which extends well lateral to the medial borders of the rectus muscles and is performed under muscular relaxation.

The authors' clinical findings lend support to my conclusion in the article entitled "Diastasis Recti: Clinical Anatomy" ${ }^{1}$ that, "contrary to current thought, abdominal wall protrusions are caused by the stretching of the entire abdominal wall and not only the linea alba."

The issue of the laxity of the musculofascial abdominal wall has been addressed at some length in my previous article on this subject, "Liposuction Abdominoplasty: An Evolving Concept." 2 In it, "the lax (or overstretched) abdominal wall" was defined as an "envelope that is larger than its contents and capable of accommodating more volume without additional stretching." In addition, in that article, a detailed description was included of my approach to preoperative and intraoperative assessment of laxity.

Because I continue to perform all my abdominoplasty procedures under local anesthesia, I have the opportunity to evaluate the laxity of the muscular abdominal wall under conditions that are as close to physiologic as possible. During surgery, laxity is assessed as the degree of tension required to approximate the rectus fascia until the abdominal wall feels tight and appears flat. I have also determined for myself a scale for laxity: grade I denotes minimal laxity, grade III denotes a very lax abdomen, and grade II is somewhere in-between.

My opinion about the intraoperative assessment of laxity differs from that of Drs. Repta and Hunstad. In my opinion, the intraoperative assessment and repair of diastasis under muscle relaxation may tend to overcorrect fascial laxity. Therefore, I had devised a segmental approach to diastasis repair, in which only the most protruding segment of the abdominal wall is repaired. I believe that this segmental approach satisfies the requirements for musculofascial repair and prevents unnecessary recurrences of abdominal protrusions.

My technique for diastasis repair is similar to that of Drs. Repta and Hunstad in that the fascial plication is confined to the anterior fascia and extends beyond the medial edges of the recti muscles. In addition, interrupted, figure-of-eight sutures (no. 1 Vicryl; Ethicon, Inc., Somerville, N.J.) in one or two layers add a vertical tightening component to the otherwise horizontal repair.

\section{References}

1. Brauman D. Diastasis recti: Clinical anatomy. Plast Reconstr Surg. 2008;112:1564-1569.

2. Brauman D. Liposuction abdominoplasty: An evolving concept. Plast Reconstr Surg. 2003;112:288-298; discussion 299-301. 


\section{Factors that May Influence Failure of the Correction of the Musculoaponeurotic Deformities of the Abdomen}

Sir:

We would like to congratulate Dr. Brauman for his excellent study published recently entitled "Diastasis Recti: Clinical Anatomy" (Plast Reconstr Surg. 2008;122:1564 -1569). The authors called our attention to the clinical aspect of the abdomen related to the width of diastasis, and they discovered that the relationship between these parameters is not always coincident. Most importantly, they verified that the correction of diastasis does not reflect a good cosmetic result in all cases, even if there is no recurrence of the diastasis with time. We completely agree with that, and we would like to add two points.

The first point is that not all deformities can be corrected surgically. The surgical correction will not be effective in cases in which the intraabdominal pressure is very high because of fat accumulation. In these cases, although diastasis may not recur, there will be an expansion of the musculoaponeuroticlayer that will become lax with time. Patients who present a weak musculoaponeurotic layer will also present projection of the abdominal contour. These patients may present an altered composition of the collagen fibers. There are two types of collagen fibers: type I and type III. Type I fibers present a higher tensile strength as compared with the type III fibers. ${ }^{1}$ Patients who present direct groin hernias present a higher number of type III fibers. Also, there is evidence that elderly patients present a higher number of type III fibers as compared with type I fibers. ${ }^{2}$ Therefore, patients present a more lax aponeurosis with age. For this reason, abdominal circumference increases and, even with surgical correction, these patients may have a poor cosmetic result. Although it may seem that plastic surgeons have limited action over the aponeurosis, it is possible to work in other areas of the musculoaponeurotic layer. We have created a classification in which four different corrections can be used to correct these deformities.3 The external oblique aponeurosis can be plicated, and this muscle can be advanced in specific deformities.

The second point is that not all patients present rectus diastasis secondary to pregnancy. Some patients (approximately 7 percent of our cases) may have a lateral insertion of the recti muscles on the costal margins: type C of our classification. 3 These patients may present hernias at the supraumbilical area and at the umbilicus. They are diagnosed by general surgeons as patients with epigastric hernias. We think that the use of "congenital rectus diastasis" is a more correct name, as not all patients present hernias. These patients may have recurrence of the rectus diastasis in the upper abdomen if the correction is made only by the plication of the anterior rectus sheath because there will occur a "violin string effect." As the recti muscles are apart from each other in their insertion, with the repeated contraction of these muscles, a dehiscence of the plication may occur. 4 If that occurs, a bulge will be formed at the superior abdomen, and the only way to correct it is to perform the entire operation again and advance anterior recti sheaths, as Dr. Brauman suggests in his article, is not the absolute way to correct abdominal wall deformities. There are other techniques that can be helpful, depending on the deformity. Also, it is important to mention that, in some cases, it is not possible to surgically correct certain deformities of the musculoaponeurotic layer.

Fabio Xerfan Nahas, MD. Ph.D.

\section{References}

1. Friedman DW, Boyd CD, Norton $P$, et al. Increases in type III collagen gene expression and protein synthesis in patients with inguinal hernias. Ann Surg. 1993;218:754-760.

2. Biondo-Simõ es Mde L, Whestphal VL, Paula JB, Borsato KS, Noronha L. Collagen synthesis after the implantation of polypropylene mesh in the abdominal wall of young and old rats. Acta Cir Bras. 2005;20:300-304.

3. Nahas FX. An aesthetic classification of the abdomen based on the myoaponeuroticlayer. Plast Reconstr Surg. 2001;108:1787-1795.

4. Nahas FX, Ferreira LM, Mendes Jde A. An efficient way to correct recurrent rectus diastasis. Aesthetic Plast Surg. 2004;28: 189-196. 


\section{Reply}

Sir:

I met Dr. Nahas briefly while giving my first presenation on this subject at the $71^{\text {st }}$ Annual Meeting of the American Society of Plastic Surgeons. His work in this field is indeed impressive.

There is evidence that the rectus muscles function moreeffectivelywhentheyareapproximated.Likewise, there is ample evidence that diastasis contributes to abdominal protuberance. Nevertheless, a diastasisis not a true ventral hernia and, aesthetically, is only visible when it is very large and/or when a person moves from lying down to standing up (and vice versa). Moreover, diastasis is merely a component of the laxity or stretching of the abdominal wall. Some of the causes of stretching and laxity are pregnancy, intraabdominal fat volume, genetic predisposition, aging, lumbar lordosis, and factors that cause an increase in intraabdominal pressure such as chronic obstructive pulmonary dis- ease, ascites, prostate hypertrophy, and others.

My first article on this subject established thatdiastasis repair does not endure if causes such as a large intraabdominal fat volume coexist. ${ }^{1}$ The present article ascertained that the presence or absence of a diastasis is immaterial because there is no linear correlation between diastasis and the degree of abdominal protuberance and that even in the absence of diastasis, abdominal protuberances occur because of the stretching of the entire musculofascial wall and not only the linea alba. ${ }^{2}$

The next article was going to describe my experience with segmental diastasis repairs, which are tapered repairs of the most protuberant segment of the abdominal wall. These repairs accomplish an aesthetic correction, decrease the chance for recurrences, and minimize the potential complications of traditional diastasis repairs. However, Dr. Nahas's comments obligate an earlier recount. Dr. Nahas is right; not all musculofascial deformities can be surgically corrected. Therefore, not only do I not perform diastasis repair on those whose deformities can- not be corrected, I also do not perform diastasis repairs on patients whom lanticipate will not significantly benefit from it. I also agree with Dr. Nahas that laxity increases with age and I believe that there is a genetic predisposition to it at all ages. I am not convinced by the evidence that typelll collagen plays a role in laxity, becauseits presence in increased amounts may represent a response to repeated injury.

Lastly, the rectus muscles diverge superiorly to beinserted into the (fifth through seventh) costal cartilages lateral to the xiphoid. Occasionally, they insert more medially into the xiphoid and in hypersthenic (more massive) individuals the subcostal angle is wide, resulting in an even more lateral insertion. ${ }^{3}$ Therefore, I plicate and taper the anterior rectus sheath repair to approximately 1 inch inferior to the xiphoid, preventing a sharp demarcation and a localized bulge, and because diastasis is not a hernia, I do not find it necessary to use rectus advancements that reinforce the diastasis but may not reduce the overall circumference because of future stretching.

DOI: 10.1097/PRS.0b013e3181a839ab

\section{Daniel Brauman, MBBS, MD}

Weill Medical College of Cornell University White Plains, N.Y.

\section{References}

1. Brauman D. Liposuction abdominoplasty: An evolving concept. Plast Reconstr Surg. 2003;112: 288-298; discussion 299-301.

2. Brauman D. Diastasis recti: Clinical anatomy. Plast Reconstr Surg. 2008;112: 1564-1569.

3. Warwick R, Williams PL, eds. Gray's Anatomy. 35th British ed. Philadelphia: Saunders; 1973:519-527, 1254-1257. 
Chapter 5.

Liposuction Abdominoplasty: An Advanced Body Contouring Technique

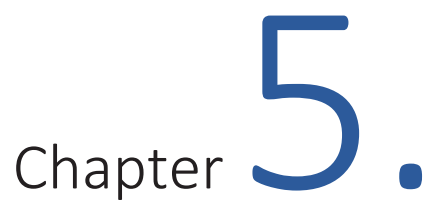

\author{
Liposuction Abdominoplasty: \\ An Advanced Body Contouring Technique
}

PLASTIC AND RECONSTRUCTIVE SURGERY VOLUME 124

Number 5 - $p$ 1685-1695

November 2009 



\section{Chapter 5. Liposuction Abdominoplasty: An Advanced Body Contouring Technique}

Plastic and Reconstructive Surgery: November 2009- Volume 124 - Number 5 - p 1685-1695

Daniel Brauman, MBBS, MD

White Plains, N.Y

From the Weill Medical College of Cornell University. Received for publication January 31, 2009; accepted June 2, 2009.

Background: Liposuction abdominoplasty was first performed by the author in 1997. In 2002, the procedure was presented as an "evolving concept" at the $71^{\text {st }}$ annual meeting of the ASPS ${ }^{1}$. Over the past 6 years an additional 294 procedures were added to the initial 43 ( $n=337$ patients), culminating in an advanced body contouring technique.

Methods: The advanced technique is characterized by a hydro-lipo-dissection technique which is selectively performed on the deep abdominal fascia, Scarpa's fascia and along the excision lines. Selective release of the skin retaining ligaments, preserves perforators and elevates a large island flap, extending from the pubis to the inframammary fold and from one flank to the other. Perforating neurovascular bundles tether the flap, minimizing dead space, and providing improved flap sensibility. Diastasis recti repair is performed utilizing newly devised segmental techniques. The flap possesses a robust circulation which enables upright patient ambulation and concomitant circumferential torso and additional areas liposuction (322 patients). Surgery is ambulatory, under local anesthesia with monitored intravenous sedation, drains are not used and patients are discharged, 2-3 hours after surgery.

Results: There were no major complications, deaths or deep vein thrombosis (DVT). Five (1.4\%) late infections of seromas responded to local drainage and six (1.7\%) marginal necroses healed without ill effect.

Conclusions: Over the past 11 years, liposuction abdominoplasty has established new concepts and evolved into an advanced body contouring technique.

\section{Introduction}

Illouz, in 1992 described a "suction abdominoplasty" technique. ${ }^{2}$ Full thickness liposuction loosened the supra-umbilical subcutaneous tissue sufficiently to allow downward advancement without undermining and when indicated, midline undermining enabled diastasis repair. Illouz also favored the creation of a neoumbilicus. The technique proved useful for those with ample loose fat in the supra-umbilical region. In 2001, Saldanha ${ }^{3}$ described his group's experience with 
Illouz's technique however when applied to all abdominoplasty candidates the technique falls short in sufficient downward flap advancement and has not gained full acceptance in the US.

In 1997 the author began performing liposuction abdominoplasty procedures, expanding the technique to all candidates and replacing traditional abdominoplasty. ${ }^{4}$ The technique introduced lipo-dissection, and a selective release of the skin retaining ligaments, while preserving many perforating vessels. The present study describes the advances in liposuction abdominoplasty made over the past 6 years and the considerable experience gained with 337 consecutive patients. The procedure is performed on all abdominoplasty candidates and enables concomitant circumferential torso and additional area liposuction.

\section{Materials and Methods}

The 337 consecutive patients included 330 females and 7 males. Their age weight and BMIs' are summarized in Table I. 324 patients underwent complete abdominoplasty and 13, a limited abdominoplasty. 15 patients underwent modified lower body lift procedures and 19, hernia repairs. 321 patients underwent concomitant circumferential torso liposuction. 105 patients underwent one additional area liposuction (eg. lateral thighs) and 44, two additional areas liposuction. 79 patients underwent buttock fat grafting, 3 underwent breast augmentations and 3 facial liposuction.

158 (47\%) patients had a hanging panniculus. 175 (52\%) had previous abdominal surgery, Table $\underline{I}, 2$ had undergone abdominal radiation for cancer and 19 had a significant smoking history.

The mean amount of fat removed per procedure (excluding the panniculus) was $2100 \mathrm{cc}$ (50$7800 \mathrm{cc})$. The mean weight of the panniculus was 384 gram and (100-3125gr.) The weight of the panniculus did not accurately represent its size since it had undergone liposuction.

Surgery was performed in an office surgical suite under local anesthesia with intravenous sedation. The usual length of surgery was 4 hours. Next day, patients walked upright, showered and returned to normal activity. Strenuous activities were avoided for 3 months when diastasis repair was done. The average follow up was 7 months ( 1 week -9 years). 


\section{$\underline{\text { Table I }}$}

Information on; age, sex, BMI.

\begin{tabular}{|lc|cc|cc|}
\hline \multicolumn{2}{|c|}{ Age (years) } & \multicolumn{2}{|c|}{ Weight (Lbs) } & \multicolumn{2}{c|}{ BMI } \\
Mean & Range & Mean & Range & Mean & Range \\
\hline 36 & $(21-72)$ & 143 & $(100-307)$ & 29.3 & $(19.5-41.7)$ \\
& & & & & \\
\hline
\end{tabular}

\section{Table II}

No. of previous abdominal surgeries, undergone by 175 (52\%) abdominoplasty patients.

\begin{tabular}{|l|l|l|l|l|l|l|l|}
\hline $\begin{array}{l}\text { No. of Previous } \\
\text { surgeries }\end{array}$ & 1 & 2 & 3 & 4 & 5 & 6 & 14 \\
\hline No. of patients & 78 & 53 & 24 & 13 & 4 & 2 & 1 \\
\hline
\end{tabular}

\section{Operative Technique}

Preoperatively, a detailed contour map is drawn, including skin creases which are the sites of the Skin Retaining Ligaments (SR-ligaments). The SR-ligaments are vertical condensations of fascia that attach the skin to the deep fascia, preventing downward advancement.

Hydro-dissection is done by the instillation of dilute anesthetic fluid using 60cc syringes and infiltration cannulas at predetermined anatomic tissue layers. First, Deep hydro-dissection is done on the deep abdominal fascia, from one flank to the other and from the inframammary crease down (over the ribcage) to the inguinal/pubic crease (ig.1a). Next, Superficial hydrodissection is performed on Scarpa's fascia from the inguinal/pubic crease to the prospective excision lines of the abdominal panniculus and somewhat superiorly, in order to bevel the inferior edge of the abdominal flap ( $\underline{\text { Fig.1b) }}$. Liposuction follows the hydro-dissections, with the objective of dissection rather than fat removal, The Deep liposuction dissects the flap free from the deep 
fascia of the abdominal wall and flanks and the Superficial liposuction dissects the panniculus away from Scarpa's fascia and bevels the lower edges of the abdominal flap. If flap thinning (below the costal margin) is necessary, it is done by additional deep liposuction of the undersurface of the flap ( $\underline{\text { Fig.1 }}$ ). Flap thickness is judged similarly to traditional abdominoplasty.

Through a curvilinear incision, mostly blunt dissection is carried superiorly on Scarpa's fascia to the umbilicus. The panniculus is bisected, and dissection proceeds inferiorly, deep to Scarpa's fascia on the deep fascia, to the pubic/inguinal crease (ig.2a,2b). Scarpa's fascia is separated from the deep fascia in the midline (for diastasis repair), and preserved laterally to add inferiorly based blood supply to the flap (Fig.2b,3). The dissection then continues superiorly from the umbilicus on the deep midline fascia to the sternal angle.

Liposuction partially separates the flap from the deep fascia by removing a layer of deep fat and by disrupting the stiff SR-ligaments with multiple holes perforations and tears while preserving the flexible vascular perforators. The flap however is still tethered to the deep fascia by the SRligaments and the neuro-vascular perforators. Therefore, it is next released by selective transection; stretching and blunt detaching of the SR-ligaments through lateral tunnels, using scissor spreading and "blind" finger dissection ( perforators which are located medial to the linea semilunaris, are spared (Fig.2b,3).

Next, traction is applied to the flap and perforating vessels that impede flap movement and "tent" the deep fascia are transected. This is done until the flap is mobile enough to reach the inferior incision with minimal tension. This stage of the procedure requires most of the experience and judgment since it is unique to each procedure. Nevertheless, as many perforators as possible are preserved.

Deep liposuction creates a layer of tissue, named "supporting tissue" by the author. The supporting tissue layer consists of a network of; blood vessels, lymphatics, nerves and collapsed connective tissue devoid of fat. It creates a distinct deep border to the flap and provides rich blood supply, lymphatic drainage and sensory nerves (Fig.3,4).

Next, when indicated, Segmental diastasis repair is performed (Fig.3,4). The term segmental diastasis repair refers to the repair of the diastasis of the most protruding segment of the musculo-fascial abdominal wall rather than a pubis to Xiphoid repair. The operating table is minimally flexed (less than 30 degrees). 

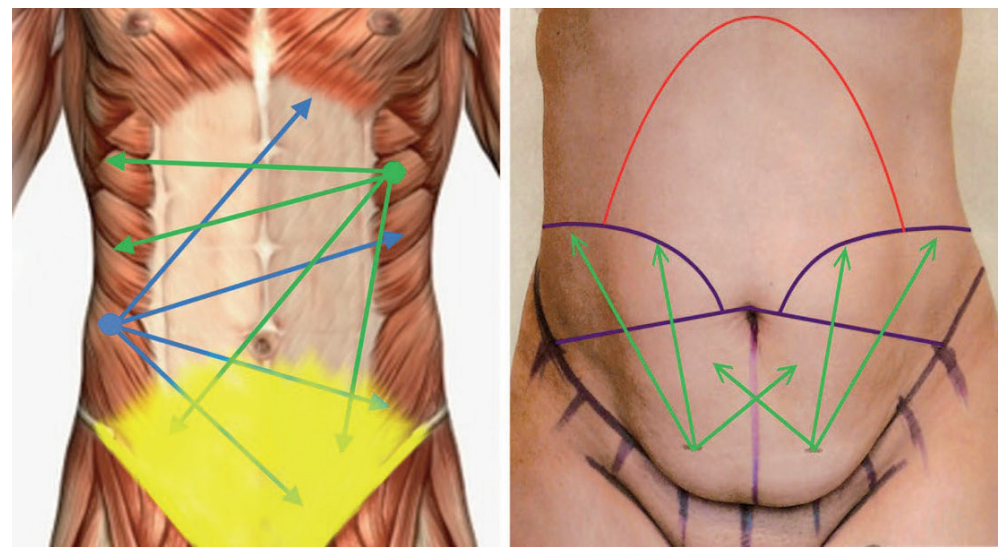

Figure 1a. Deep Lipo-Dissection. Done from the right side; lateral and just superior to the umbilicus - Blue arrows, and the left upper side - Green arrows. The dissection stays on the deep fascia inferior to the umbilicus, since the downward direction allows the cannula to "slip" deep to the upper edge of Scarpa's fascia - Yellow. Figure 1b. Superficial Lipo-Dissection. Done from two stab wounds near the inguinal crease - Green arrows. The upward direction from the crease directs the cannula to remain superficial to Scarpa's fascia which is densest at the inguinal crease. The dissection bevels the inferior edges of the abdominal flap up to the curved Blue lines. The straight Blue lines diverging from the umbilicus are the inferior edges of the abdominal flap. No liposuction into the flap is done below the Red line (costal margin).
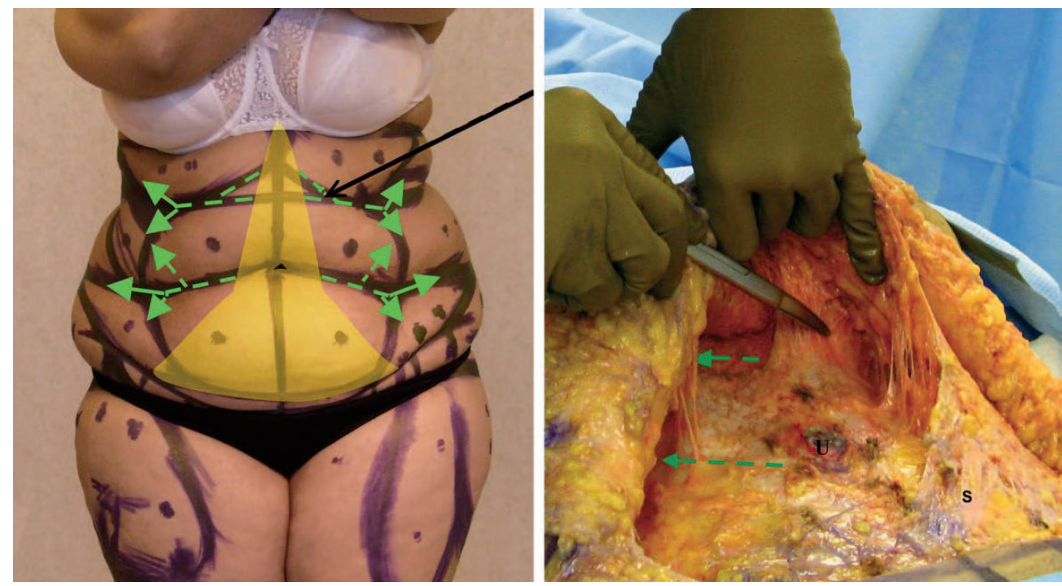

Figure 2a. Contour map of abdomen. Skin creases, marked with a purple marker, are the sites of the Skin retaining ligaments. The extent of the continuous flap release (undermining) on the deep fascia and Scarpa's fascia is highlighted in Yellow. The extent of lateral tunnels selectively releasing the skin retaining ligaments while preserving perforators (discontinuous undermining) is marked with Green arrows. The tight superior crease makes this patient an unsuitable candidate for Illouz's technique.

Figure $\mathbf{2 b}$. Intraperative photo of the patient in Figure $\mathbf{2 a}$. Green arrows point into the openings of right sided tunnels of selective release. The scissors point to the skin retaining ligament marked by a Black arrow in Figure 2a. Left sided release has not yet been done. $\boldsymbol{U}$-Umbilicus. $\boldsymbol{S}-\mathbf{S c a r p a}$ 's fascia has been preserved laterally. 


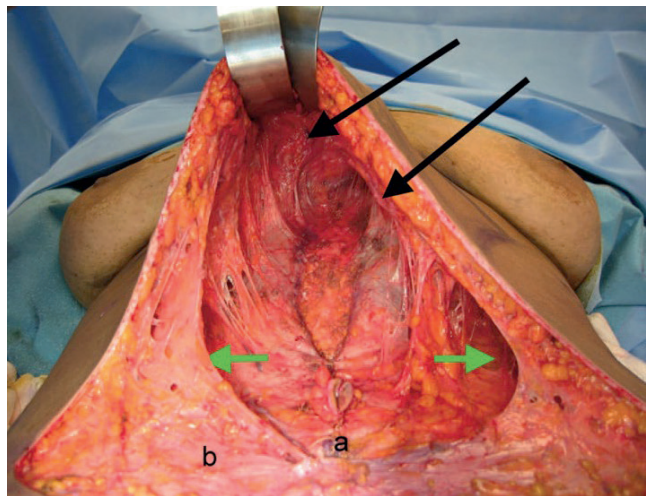

Figure 3. Supporting tissue; Segmental diastasis repair of the infra-umbilical diastasis (a) (and one inch of the supra-umbilical diastasis) is completed. Scarpa's fascia (b), was able to be preserved. It covers the lower portion of the repair and provides additional circulation to the flap laterally. The panniculus was excised leaving an even thickness flap. Black arrows point to a layer of supporting tissue lining the flap. Green arrows point to selective release tunnels. Release of the tissue over the ribs (and the costal margins) was not necessary in this patient with loose epigastric tissue and ligaments, suitable for Illouz's technique.

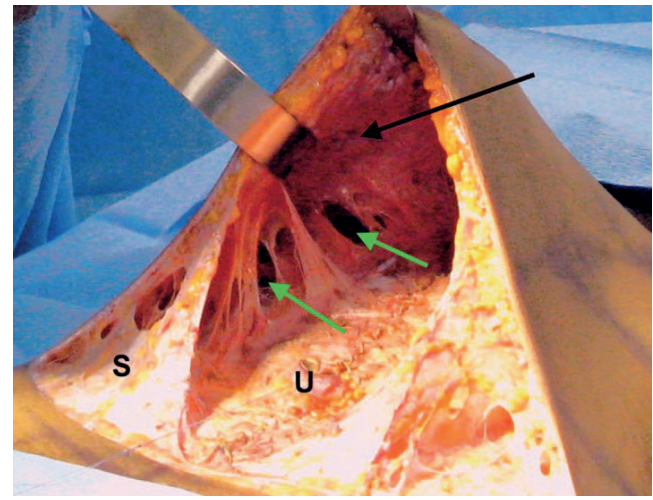

Figure 4. SR-ligaments and supporting tissue; Repair of 4" of the supra-umbilical and 2" of the infra-umbilical diastasis corrected the protrusion of the central abdomen. Green arrows point to the opening of lateral tunnels where SR ligaments were bluntly released. Black arrows point to bluish veins within the supporting tissue layer. This patient was thin (BMI: 18) and her tissues exceptionally tight and fibrous, not a candidate for Illouz's technique. $\boldsymbol{U}$; umbilicus. S: Scarpa's fascia.

The bisected panniculus is then excised, bleeding from the edges assessed, and the lower abdominal incision is closed with interrupted suture/staple closure. Tension in the midline is judged appropriate when flap blanching is not present and a 3:0 Vicryl suture (Ethicon) can approximate the flap unaided by the assistant.

The patient is then rotated onto the right and left sides, circumferential torso liposuction and other area liposuction is done. The lateral edges of the incision are closed. No drains are used.

\section{Results}

There were no deaths, major complications, anesthesia complications or DVT. One patient was transfused a few days after surgery when Plavix was prematurely resumed by her cardiologist. There were 5 infections (1.4\%), occurring several weeks after the surgery as a result of seromas becoming infected. The infections, attributed to the use of drains prior to 2005 , responded to simple drainage. There were 6 cases of minor tissue necrosis (1.7\%). All healed without affecting the final result. The 6 patients had significant smoking histories. 2 involved T-junctions and 2 were caused by drain site pressure. Both, drains and T-junctions have not been used since 2005 . 
2 more recent cases were caused by attempts to extend the inferior reach of the flap despite a high umbilicus and a tight epigastrium. 2 additional cases of tissue loss occurred in smokers, in whom concomitant repairs of umbilical hernias were done. The hernia repairs endured and the umbilical scars were subsequently sutured to the fascia. Patient satisfaction was high as evidenced from satisfaction surveys and patient referrals.

\section{Discussion}

Traditional abdominoplasty entails undermining to the costal margin, table flexion, closure of incision and an assumption that flap circulation will withstand the subsequent tension, even when a subcostal incision is present. ${ }^{5,6,7,8,9}$ In contrast, liposuction abdominoplasty introduces a new dissection technique (not undermining), which enhances blood supply, evaluates, not assumes flap circulation, utilizes new methods for patient evaluation and establishes new concepts in abdominoplasty.

Tension is the single most important cause of flap necrosis. Therefore, a balance needs to be struck between flap release and blood supply and a clear understanding of the clinical anatomy is a prerequisite.

The circulation to the supra-umbilical abdomen originates from the lateral and anterior branches of T, 7-10 intercostal vessels anastomosing with the deep epigastric vessels. T, 7-8 vessels cross the costal margin deep to the rectus muscle and T, 9-10 also enter the deep surface of the rectus and a pierce it medially. Therefore, undermining (especially discontinuous) beyond the costal margin does not devascularize the flap. The infra-umbilical circulation originates from T,11-L,1 anastomosing with the deep epigastric vessels and from the superficial epigastric and circumflex iliac vessels which travel superficial to Scarpa's fascia. Therefore, preservation of Scarpa's fascia/vessels laterally adds additional inferior circulation to the flap.

Camper's and Scarpa's fasciae are well described horizontal layers of the abdominal fascia, however the vertical layers of the fascia had not been described prior to the author's description (in 2002) of the Skin Retaining and their clinical significance. ${ }^{1,4}$ These ligaments are vertical condensations of the fascia that tether the skin to the deep fascia and their transection allows the downward advancement of the flap without tension. Therefore, the disruption of the ligaments by liposuction aided by lateral, blunt finger and spreading scissor dissection releases the flap and reduces tension. 

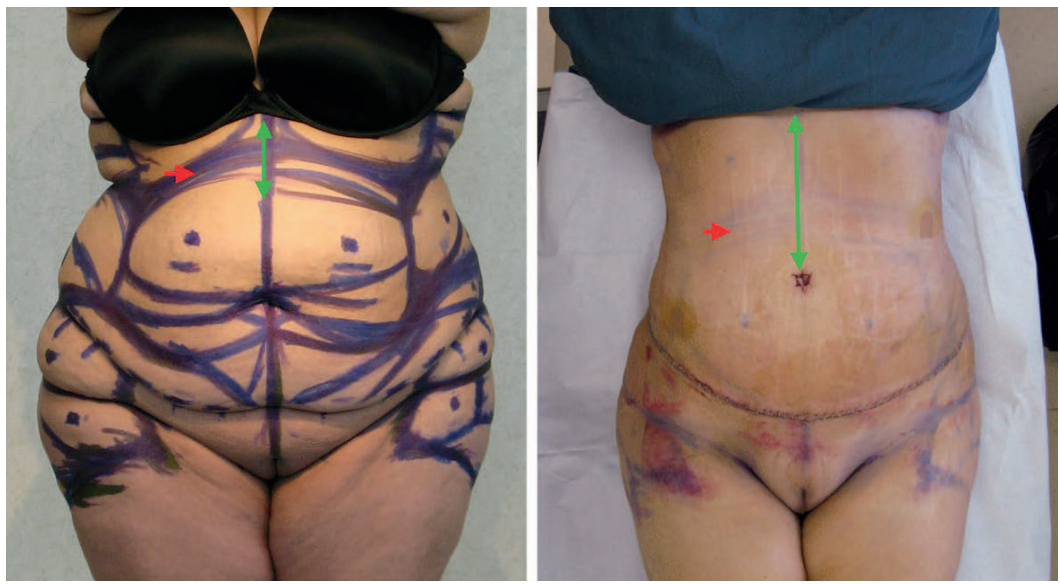

Figures 5 a \& b. Retaining ligament release; Before and after photos of 37 year old, (202 lb., BMI: 37, abdominal liposuction: $2800 \mathrm{cc}$, total lipoaspirate: $5800 \mathrm{cc}$, panniculus: 1200gr). The after photograph was taken the morning after surgery with the patient supine (flat). The photograph was rotated upright for ease of comparison. The Green arrows demonstrate the extent of the midline stretch enabled by the release of the tight superior transverse crease/ligament, marked with a Red arrow, (still faintly visible in the post operative photo).

Anatomically, the SR ligaments are distinct from the perforators therefore their selective transection does not impair the blood supply. They are visible on the surface as skin creases and depressions with constant locations. The superior transverse crease corresponds to the first tendinous intersection of the rectus muscle and the costal margin and the inferior transverse crease corresponds to the third intersection near the umbilicus. The two vertical depressions on either side correspond to the lateral edge of the external oblique aponeurosis and the linea semilunaris (Fig.2a)

A balance between tension and circulation is achieved during surgery, when selected ligaments are disrupted or released and later; while traction is applied to the flap, selected perforating vessels are transected if they impede flap movement. The rule is; tension trumps circulation. That is; perforators are transected to allow closure with minimum tension (Fig.2a,5a,b).The extent of undermining is less than with traditional abdominoplasty and varies from procedure to procedure. The flap will tolerate wide undermining, it will not tolerate excessive tension or a hematoma.

Illouz's "suction abdominoplasty" technique ${ }^{2}$ utilized liposuction of the entire thickness (except for the most superficial $1 \mathrm{~cm}$ ) of the supra-umbilical subcutaneous tissue to create tunnels that loosened the flap sufficiently to allow inferior advancement. Illouz's technique was (and is) only suitable for those who had loose and (frequently) obese supra-umbilical tissues and in whom liposuction tunnels and naturally slack SR-ligaments allowed downward flap advancement (ig. 3 ). 
However in those whose supra-umbilical tissues are tight or thin and whose ligaments are tight, sufficient advancement is not possible without ligament release (Fig.2a, 2b, 4,5a,b).

Saldanha ${ }^{3}$, in 2000, expanded Illouz's technique to all abdominoplasty candidates. However, the photographs presented in his article show high incisions with insufficient downward flap advancements and in the author's opinion, an attempt to extend the downward reach of the flap to the pubic crease would have resulted in a fairly high incidence of flap necrosis.

Brauman, in 1997 started performing his "liposuction abdominoplasty" technique. The initial published technique ${ }^{4}$ employed entire thickness liposuction similar to Illouz's with four major points of difference. Deep lipo-dissection identified the SR-ligaments and perforators and release (blunt detachment and stretching) of the skin retaining ligaments was performed while selectively preserving the perforators. Liposuction within the flap avoided an area superior and lateral to the umbilicus and the panniculus was separated from the flap with liposuction which defatted and beveled the deep surface of the flap's inferior edges. This extended the lower reach of the flap to the pubic crease; however $T$ junctions were still necessary when the upper abdominal tissues were exceptionally tight.

With experience gained during the past six years, the author enhanced his technique and extended the downward reach of the flap by more extensive lateral release, which reduced tension. The blood supply was enhanced by limiting liposuction to the deep layer and creating a supporting tissue layer and by preserving Scarpa's fascia. In the areas of the flanks and back, liposuction disrupts the ligaments and reduces the appearance of skin creases and fat rolls (Fig.6a,b). However, since there is more extensive body contouring and release than before, a greater theoretical potential for seromas exists and meticulous hemostasis is exercised in order to prevent hematomas. Nevertheless, the well vascularized flap enables safe circumferential torso liposuction and as well as immediate upright ambulation (Fig.7a-d, 8a-d).

The precise use of liposuction (not ultrasonic or laser) as a dissection tool is preferred to the use of cautery or scalpel, since it causes less vascular damage to the flap and less bleeding than undermining. It selectively disrupts the stiff ligaments while preserving the flexible perforators and it enhances flap circulation by creating a vascular supporting tissue layer, made of a rich network of blood vessels, lymphatics, connective tissue and nerves. The supporting tissue layer also provides a finite deep layer devoid of devascularized fat and assures even flap thickness and deep de-fatting without surface irregularities. 

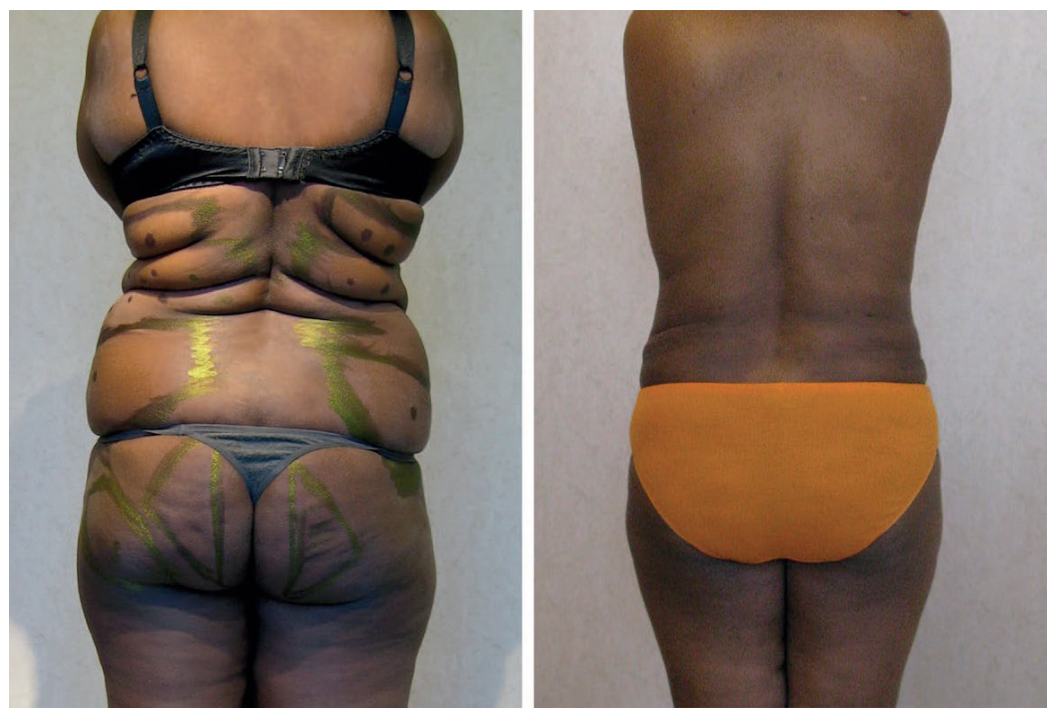

Figures 6 a \& b. Skin retaining ligaments of back; Before and after photos of liposuction of back, hips and waist, demonstrating disruption of the skin retaining ligaments and improvements to the appearance of skin creases and fat folds.

A complete discussion of Diastasis repair is not the scope of this paper. Briefly, the author believes that diastasis repair contributes to DVT and postoperative respiratory complications because it increases intra-abdominal pressure. Moreover, the author has established ${ }^{4,10}$ that diastasis will recur and/or the abdominal wall will re-stretch if musculo-fascial laxity is not present due to an increase in intra-abdominal volume/pressure. Therefore, a tapered repair of (only) the most protruding segment of the diastasis (segmental repair) is performed. This provides a good cosmetic result and has prevented recurrences and complications (Fig.3,4).

The preoperative evaluation of a surgical candidate is done standing up. The height of the umbilicus and the thickness and looseness of the skin and subcutaneous tissue are determined (Fig.9a,b). Scars affect tissue mobility and/or circulation and their internal retraction and external straightening predict healing. Muscle protrusion and intra abdominal fat volume are evaluated for diastasis repair and because a large/tense epigastric protrusion will hamper flap mobility. The mons pubis is evaluated for correct incision placement.

The percentage of complications in this study compares favorably with recent data from TOPS and CosmetAssure. ${ }^{11}$ The 5 late infections reported, occurred prior to 2005 when drains were used, as a result of seromas which became infected several weeks after surgery. Smoking, obesity 
and T junctions were predisposing factors. ${ }^{9}$ Reverse isolation techniques practiced since 1982 have been effective in that the organisms have always been "community based"/ non resistant.

The reported incidence of seromas varies widely. This seems to be due to differences in definition since seromas are defined as fluid collections occurring after drain removal and the time to drain removal varies widely from patient to patient and author to author. ${ }^{12}$ In this study seromas occurred more frequently in patients with high BMI's, massive weight loss, and following diastasis repair. It also seems that an individual predisposition to seromas exists, since histories of previous seromas were elicited in some patients. Moreover, the author had explored drain sites weekly after drain removal and drained small fluid collections for up to six weeks after surgery when clinical examination failed to detect them. Therefore, seromas were defined as fluid collection persisting (in 5 patients since 2005) beyond the "normal" six-week interval. Consequently, no drains were used after 2005 and periodic tapping is performed. The author is presently exploring the effect of systemic or instilled steroids since he views seromas as an expression of the inflammatory wound healing process.

The author judges the magnitude of the liposuction by the total input and output of anesthetic (and IV) fluids rather than the volume of lipoaspirate. ${ }^{13}$ The author has been performing procedures under local anesthesia with sedation since 1982. An acute care nurse administers medications under the surgeon's supervision. ${ }^{14,15,16,17}$ Over the past 26 years, several thousand procedures have been safely performed without any anesthetic complications or DVT/PE. Despite changes due to the advent of monitoring instrumentation and medications such as Propofol and Ketamine, two salient principles remain; accurately monitoring respiration without supplemental oxygen, and that the principal drugs used are reversible by antagonists. The performance of surgery under local anesthesia and sedation requires extensive training, experience and a special interest in medicine and acute care. 

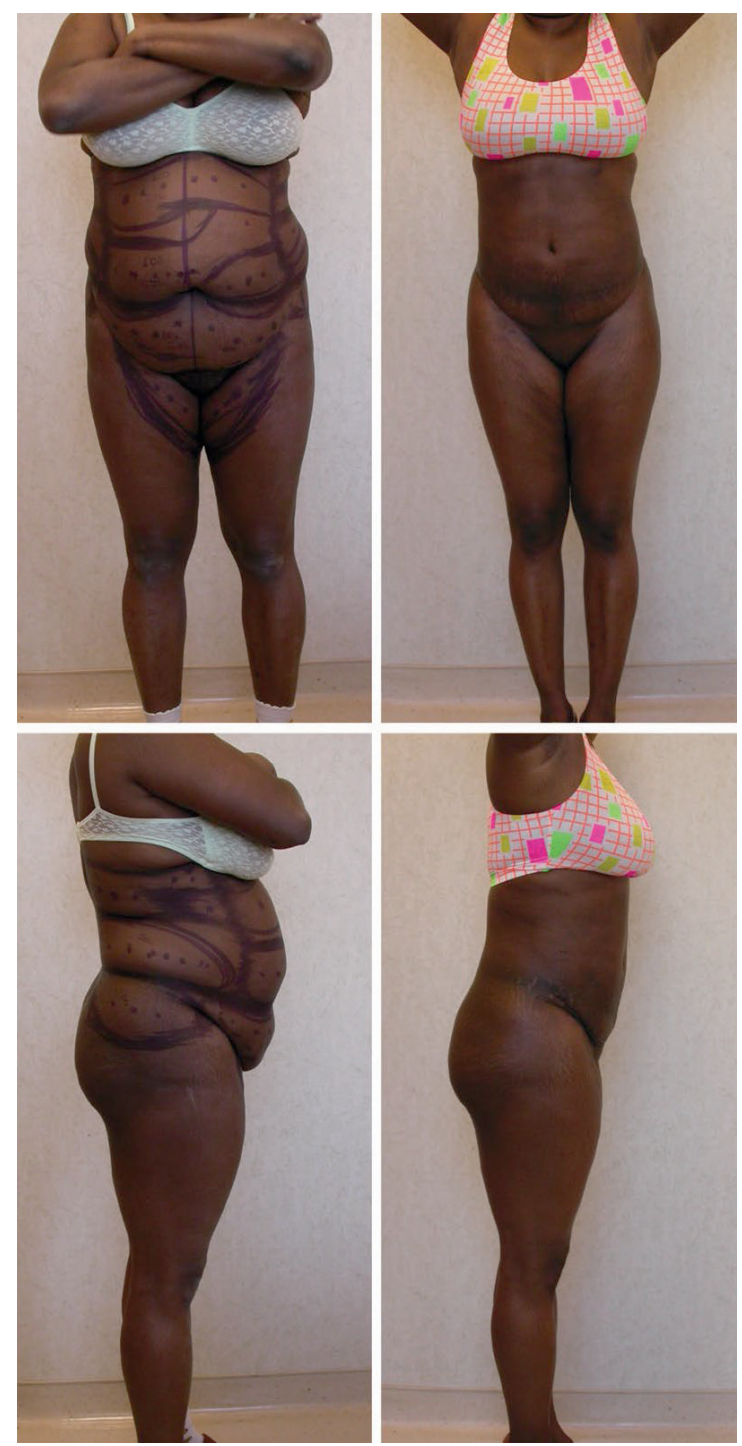

Figures 7 a-d. Before, and 3 weeks after photos, of a 43 year old who underwent liposuction abdominoplasty and torso liposuction. (Abdominal fat: 2500cc, panniculus: $500 \mathrm{gr}$ ). The contour map includes the skin retaining ligaments. The incision was placed in the crease, at the junction with the pubis. The depressed and hyper-pigmented area above the pubic area is the result of girdle compression and irritation. The incision extends only minimally to the side. 

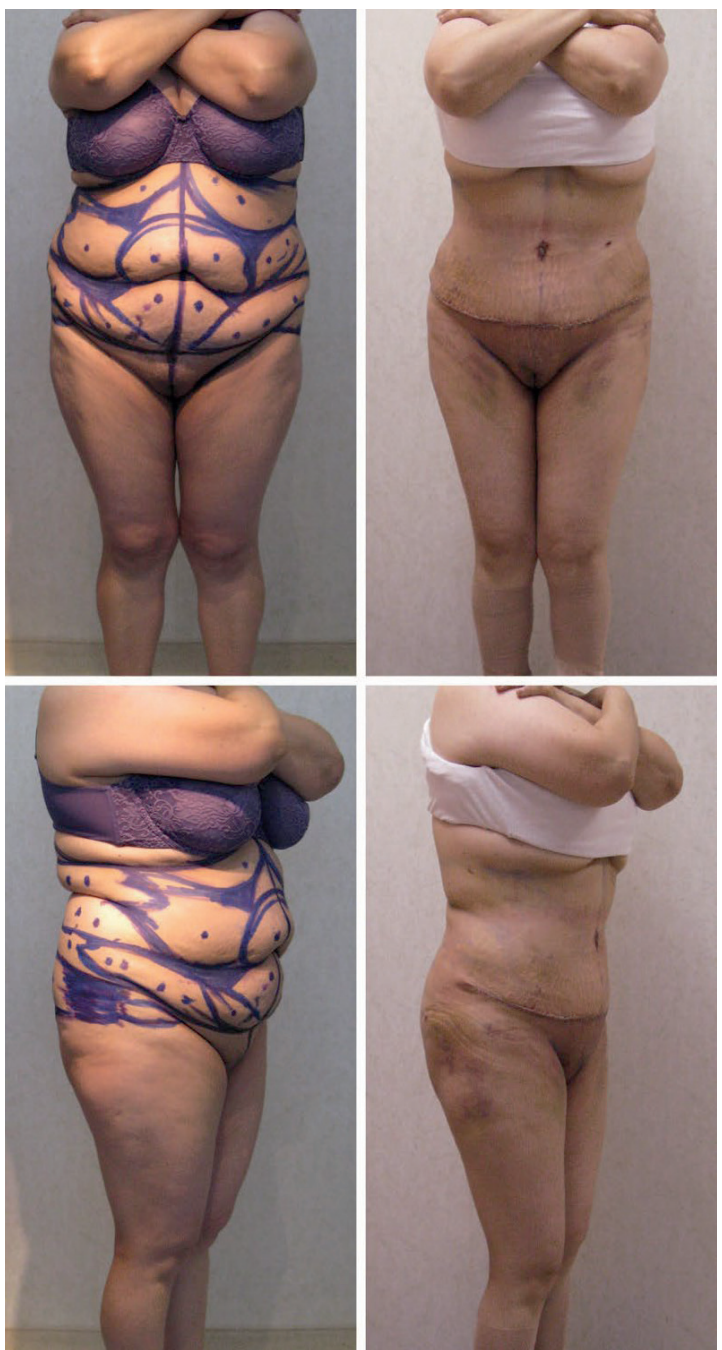

Figures 8 a-d. Before, and 1 week after photos, of a 53 year old who underwent liposuction abdominoplasty and torso liposuction. Abdominal fat: 1500cc, total lipoaspirate: $4750 \mathrm{cc}$, panniculus: $850 \mathrm{gr}$ ). The patient is seen for staple removal. The contour map includes ligaments to be divided or disrupted. Diastasis repair was not judged necessary and therefore not performed. The abdomen is flat and the patient has resumed normal activity. The deep creases of her waist and back show remarkable improvement due to disruption of the ligaments, and the buttocks are better shaped. 

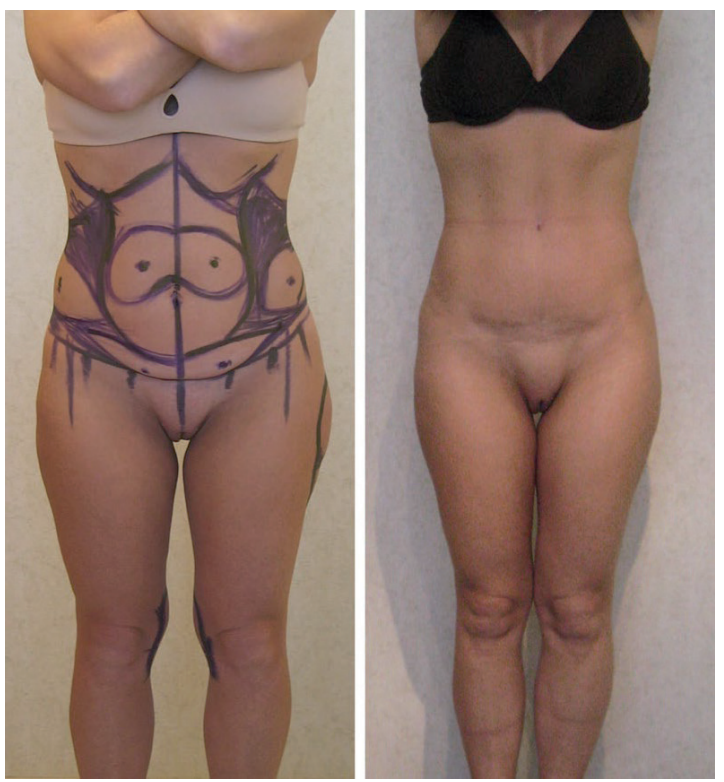

Figures 9 a \& b. Before and 1 year after photos of 39 year old who underwent liposuction abdominoplasty with liposuction of the hips and waist (100lb, BMI: 19.5, abdominal fat: $100 \mathrm{cc}$, total lipoaspirate: $375 \mathrm{cc}$, panniculus: $350 \mathrm{gr}$ ). This abdominoplasty presented a challenge because of tight and thin supra-umbilical tissues and the extensive ligamentous attachments to the deep fascia laterally. It is impossible to advance the tissues using Illouz's technique and even with the author's technique surgery is difficult to execute.

\section{Conclusion}

Liposuction abdominoplasty is not only a technique; it introduces many new concepts of tension, circulation, clinical anatomy, SR-ligaments, lipo-dissection, selective release and disruption, supporting tissue layer, deep de-fatting, diastasis repair and patient evaluation.

The enhanced abdominoplasty results and improved safety of the abdominoplasty component enables dramatic body contouring with less morbidity and a fast return to normal activity Liposuction abdominoplasty is reproducible with the caveat that beginners should be conservative with the extent of downward flap advancement. 


\section{References}

1. Brauman, D. Liposuction abdominoplasty: an evolving concept. Presented at the $71^{\text {st }}$ annual meeting of the ASPS. San Antonio, Texas. Nov. 6, 2002.

2. Illouz, Y-G. A new safe and aesthetic approach to suction abdominoplasty. Aesth. Plast. Surg. 1992;16:237-245.

3. Saldanha, O. R., de Souza Pinto, E. B., Matos, W. N., Jr., Lucon, R. L., Magalhães, F., and Bello, É. M. L. Lipoabdominoplasty without undermining. Aesthetic Surg. J. 2001; 21: 518.

4. Brauman, D. Liposuction abdominoplasty: an evolving concept. Plast. Reconstr. Surg. 2003;112: 288298.

5. Pitanguy, V. Abdominal lipectomy: An approach to it through an analysis of 300 consecutive cases. Plast. Reconstr. Surg. 40: 384, 1967.

6. Regnault, P. Abdominoplasty by the W technique. Plast. Reconstr. Surg. 55: 265, 1975.

7. Regnault, P. Abdominal dermolipectomies. Clin. Plast. Surg. 2: 411, 1975.

8. Wilkinson, T. S., and Swartz, B. E. Individual modifications in body contour surgery: The limited abdominoplasty. Plast. Reconstr. Surg. 77: 779, 1986.

9. Shermak, M.A., Mallalieu, J. E., Chang, D. Do pre-existing abdominal scars threaten wound healing in abdominoplasty. Plast. Reconstr. Surg. 2008; 122 (4) : 62.

10. Brauman D. Diastasis Recti: Clinical Anatomy. Plast. Reconstr. Surg. 2008, 112;5; 1564-1569.

11. Alderman, A. K. et al. Benchmarking outcomes in Plastic Surgery:National complication rates for abdominoplasty and breast augmentation. Plast. Reconstr. Surg. 2008; 122 (4) : 5-6.

12. Rezak, K. M., Borud, L. J. Reducing seromas: Lipoabdominoplasty with lower body lift in the massive weight loss patient. Plast. Reconstr. Surg. 2008; 122 (4) : 61.

13. Brauman D. Lipoplasty: A Case for a Low-volume Procedure. Aesthetic Plastic Surgery. 2000; 20:5:373-380.

14. Motura, A. A. Local anesthesia for abdominoplasty, liposuction and combined operations. Aesth. Plast. Surg. 17:117,1993.

15. Kryger, Z. B., Fine, N. A., Mustoe, T. A. The outcome of abdominoplasty performed under conscious sedation: A six year experience in 153 consecutive cases. Plast. Reconstr. Surg. 2004; 113:18071817. 
Chapter 5

16. Brauman, D. Liposuction: The case for a low volume procedure. Manuscript \# 99-ma1023. Submitted to Plast. Reconstr. Surg. 11/4/1999.

17. Evans, J. Propofol sedation for endoscopy faces obstacles. Surgery News. 2005; 1:19. 
Chapter 6.

A Case for Local Anesthesia and Prevention Protocols for Curbing DVT in Abdominoplasty

Chapter

\author{
A Case for Local Anesthesia \\ and Prevention Protocols \\ for Curbing DVT in Abdominoplasty
}





\title{
Chapter 6.
}

\section{A Case for Local Anesthesia and Prevention Protocols for Curbing DVT in Abdominoplasty}

\author{
Daniel Brauman, MBBS, MD \\ Rene R.W.J. van der Hulst, MD, Ph.D \\ and Berend van der Lei, MD, Ph.D
}

\begin{abstract}
Background: Abdominoplasties performed under general anesthesia carry a significant risk for venous thromboembolism (VTE). Abdominoplasties under local anesthesia with sedation have been reported to have a much lower risk for this complication. This study seeks to determine if the performance of abdominoplasties under local anesthesia with sedation reduces the risk for deep venous thrombosis (DVT) and pulmonary embolism (PE).

Methods: Relevant data on abdominoplasties performed under local and general anesthesia was retrieved and compared with regard to the incidence of VTE and anesthesia complications. Surgical complications and unsatisfactory aesthetic results were excluded.

Results: The overall risk of VTE in abdominoplasty under general anesthesia varies and ranges from 0.067\% -1.1\%. Chemoprophylaxis in abdominoplasty remains controversial. The risk of VTE increases with more extensive and/or combined procedures. Abdominoplasty under local anesthesia with sedation appears to prevent the risk of VTE without an increase in anesthetic complications.

Conclusions: The terms DVT and PE accurately describe thrombosis and embolism. The abbreviation VTE interferes with the accuracy of reporting and therefore ought to be replaced by the abbreviation DVT/PE. Local anesthesia with sedation likely prevents DVT/PE by reducing venous stasis. DVT prevention protocols are effective when used in their entirety.
\end{abstract}




\section{Introduction}

Highly publicized deaths in the US during the 1990's led to requirements mandating the reporting of adverse events in office-based surgery. In 2012, 10-year data from Florida revealed that $56.5 \%$ of deaths and $49.8 \%$ of hospital transfers were associated with cosmetic procedures. Of those, $67 \%$ of all deaths and $74 \%$ of all hospital transfers due to cosmetic surgery were done under general anesthesia. Plastic surgeons reported $45 \%$ and dermatologists reported $1.3 \%$ of all complications and no deaths. 6-year reporting from Alabama yielded similar results. This data was reported as "Cosmetic surgeries performed under general anesthesia accounted for the vast majority of deaths in Florida, with liposuction and abdominoplasty the most frequent procedures" and "No deaths occurred in the setting of local anesthesia." ${ }^{1-3}$

This study aims to correlate the risk of DVT with the use of general anesthesia (general) or local anesthesia (local) in abdominoplasty, and to find out if an expected reduction of DVT risk with local anesthesia comes at the expense of an increased incidence of anesthetic or post-anesthetic complications.

\section{Patients and Methods}

Relevant data from an extensive search of the literature was selected and cited.

Grazer and Goldwyn 1977 survey of 10,490 abdominoplasties under general anesthesia yielded a DVT incidence of $1.1 \%$, a pulmonary embolus (PE) rate of $0.8 \%$, deaths due to pulmonary emboli (PE) $0.057 \%$, and an overall death rate of $0.16 \%{ }^{4}$

Teimourian and Rogers 1989 surveyed 112,756 body contouring cases that included 26,562 Abdominoplasties performed from 1984 to 1988 under general anesthesia. The 26,562 abdominoplasties sustained $0.29 \%$ DVT, $0.24 \% \mathrm{PE}$, anesthetic complications $0.15 \%$, death from PE $0.022 \%$, and overall deaths $0.041 \%$. Furthermore, pulmonary embolism was the cause of $60 \%$ of the deaths in the 112,756 body contouring cases reviewed that included; liposuctions, nonabdominal dermolipectomies, and abdominoplasties. ${ }^{5}$ 
Motura 1993, described his experience with 25 abdominoplasties under local anesthesia with sedation without DVT/PE or anesthesia complications. ${ }^{6}$

Nguyen 1997, reported 35 outpatient mini-abdominoplasty surgeries utilizing tumescent lidocaine and oral diazepam or intravenous versed sedation without complications. ${ }^{7}$ Pravecek 1998, described performance of full abdominoplasties since 1996 under local anesthesia with sedation at his office surgical suite. ${ }^{8}$ Abramson 1998, shared his experience with 6 tumescent abdominoplasties under sedation, performed at his office suite with an anesthesiologist. ${ }^{9}$ Shestak 1999, related his experience with a -marriage limited abdominoplasty concept performed under local anesthesia and deep sedation. ${ }^{10}$ Brauman 2000, described office-based abdominoplasty performed under local anesthesia with surgeon-directed sedation since $1996 .{ }^{11}$ Kryger et al. 2004, conveyed his groups' experience with abdominoplasties under surgeon directed conscious sedation at their hospital's ambulatory department since $1996 .{ }^{12}$

Hatef, Kenkel et al. 2008 evaluated 86 abdominoplasties, not excluding liposuction 2003-2006 under general anesthesia. VTE incidence was $4.65 \% .^{13}$

Hatef, Trussler and Kenkel in 2010 evaluated 4606 abdominoplasties selected from 30 articles representing a literature search of 114 articles. ${ }^{14}$ The term VTE was chosen to replace DVT and PE to streamline the statistical analysis. The incidence of VTE in 1189 abdominoplasties was $0.34 \%$, the incidence in 2381 abdominoplasties with liposuction and/or other procedures was $0.67 \%$, in 507 abdominoplasties with intraabdominal procedures $2.17 \%$, and in 529 circumferential abdominoplasties $3.4 \%$. The Hatef article did not specify the type of anesthesia used in the 30 articles reviewed still, 4 of the 30 articles cited included, comprising 313 abdominoplasties, were performed under local anesthesia with sedation with a VTE incidence of $0 \%$ and no anesthesia complications. ${ }^{15-18}$

Wincour et al.2017. Reviewed the 2008 - 2013 CosmetAssure database. 8975 abdominoplasties were examined and the incidence of VTE was $0.20 \%$. Keyes's commentary of the article quoted 
similar data from the 2001 - 2011 American Association for Accreditation of Ambulatory Surgical Facilities (AAAASF) database. The study did not qualify the type of anesthesia used. ${ }^{19,20}$

Brauman, van der Lei, van der Hulst 2018, presented 600 consecutive prospectively studied abdominoplasties under local anesthesia with sedation (1996-2018) without DVT/PE or anesthesia complications. ${ }^{21,22}$

Sozer et al. 2018, reported a series of 1000 consecutive abdominoplasties under general anesthesia with enoxaparin prophylaxis. DVT $0.015 \%$ death $0.001 \%{ }^{23}$

\section{Results}

The roughly 52,389 abdominoplasties cited were a fraction of an extensive literature search that could not provide some of the details sought in this study. With a few notable exceptions (Teimourian), the studies targeted topics such as risk stratification, chemoprophylaxis, etiology and prevention. Data retrieval was also encumbered by overlapping time periods, different databases and more recent data that included the use of the term VTE to describe DVT and/or PE. Furthermore, the generally low incidence of DVT and PE makes the determination of statistical significance difficult.

The risk of DVT/PE in abdominoplasty under general anesthesia ranged from $0.067 \%$ to $1.1 \%$. The risk increased with extensive circumferential procedures, added body regions, invasion of body cavities, added procedures, and predisposing health issues. Chemoprophylaxis in abdominoplasty remains controversial because of inconsistent results, different protocols, a variety of anticoagulants and the potential for bleeding complications. ${ }^{14,23,24}$ Nevertheless, abdominoplasty under local anesthesia with sedation appears thus far to prevent the risk of DVT/PE without an increase in anesthetic complications. 


\section{Discussion}

Deep vein thrombosis is the clotting of pooled blood in the deep venous system and as such, its causes are 1 . Venous stasis 2. Hypercoagulability and 3. Injury to vessel wall. Therefore, a systematic approach to DVT prevention is the identification, grading, and elimination of its causes.

\section{DVT Prevention Protocols}

The DVT protocols discussed below have proven effective in preventing DVT in the cited 600 consecutive abdominoplasties with circumferential torso liposuction (that included 52 massive weight loss patients), and in several thousand additional body and facial contouring cases since 1982, and although many of these protocols are straight forward, well known, and widely practiced, the prevention of DVT lies in the steadfast adherence to the -entirety of the measures described. ${ }^{22}$

\section{Local anesthesia}

The absence of reported cases of DVT in abdominoplasty (thus far) under local anesthesia supports the belief that local anesthesia appears to be the most effective DVT prevention protocol. Venous return is controlled by the muscle pump, the respiratory pump and cardiovascular function. General anesthesia reduces muscle tone (pump) and positive pressure ventilation lessens the effectiveness of the respiratory pump thereby reducing venous return. Sedation better maintains muscle tone and enables the evaluation of abdominal laxity under truer physiologic conditions, preventing abdominal wall overtightening and a resultant increase in intraabdominal pressure and respiratory dysfunction. ${ }^{25,26}$ Diastasis repair was only undertaken if sufficient laxity was confirmed intraoperatively and if the individual was deemed medically low risk, ideally with a small intraabdominal fat volume. Segmental diastasis repairs aim to further reduce the intraabdominal pressure by only repairing the segment protruding the most, rather than the entire diastasis. ${ }^{27-29}$ 


\section{Protocols}

Additional DVT prevention protocols include. - Patient ambulation on and off the operating room (OR) table. -Surgeon positioning of patients on the OR table with elevated legs and slightly flexed knees. -Continuous active and passive ankle flexions and extensions to exercise the calf muscles performed by the patient when awake (with frequent prompting by facility staff in recovery, and family when at home), and performed intra-operatively by a nurse every 15-20 minutes in addition to -Intermittent compression devices. Periodically prompting deep breathing (respiratory pump) -Minimizing invasive monitoring such as urinary catheters. -Hydration and voiding at least twice post operatively until clear. -A dressing technique that eliminates groin compression. Ambulatory surgery - Prohibition of car or any other travel in excess of 20-30 minutes for 14 days postoperatively.

\section{Patient and Family Education}

Patient and family education by surgeon and staff include preparing the patient and family prior to surgery with emphasis on DVT avoidance. Repetition of instructions, emphasis on ankle flexion and extension, deep breathing, and walking every hour on the hour until midnight the night of surgery. Avoidance of travel and avoidance of sitting and dependent leg positions. Insistence on walking, getting out of the house, resumption of non-strenuous activities the day after surgery. Daily showers and dressing changes. Frequent post-operative follow-up visits. Phone calls to patients with encouragement and verification of compliance by patients.

Maintaining a close and personal relation with the patient and family is crucial and there needs to be a willingness to spend the time and to do it tactfully. In addition to the sense of safety imparted, the patient and caretakers are thereby recruited to join and assume an active role in their own care.

\section{Medications}

Aspirin, $81 \mathrm{mg}$ daily plus Vitamin E, $15 \mathrm{mg} /$ day, is begun on the 2nd post-operative day and continues for one month. The role of anticoagulants in abdominoplasty remains to be determined because the establishment of standards have been delayed by the use of different 
anticoagulants and administration routines, bleeding complications and inconsistent outcomes. ${ }^{14,23,24}$

\section{Venous Sonograms}

Ordered at the slightest suspicion of DVT.

\section{Local anesthesia and sedation}

Abdominoplasty under local anesthesia with sedation is contingent on an effective local anesthesia/numbing technique. The local anesthetic and sedation techniques are clinically based and have been shown effective and safe..1,22 The muscular abdominal wall is effectively anesthetized with a dilute lidocaine infiltrate of $0.056 \%$ lidocaine. Moreover, the subsequent addition of an epinephrine only infiltrate afterwards was found not to decrease the effectiveness of the lidocaine infiltrate. The use of an epinephrine infiltrate as an additional wetting solution and the sequential infiltration of the lidocaine all through the surgery keeps down the amount of lidocaine instilled per unit of time. Furthermore, tumescing the skin, provides skin anesthesia without the need to tumesce the entire subcutaneous tissue.

Despite the advent of improved monitoring devices and the development of new medications such as Propofol, three basic tenets were kept: monitoring of blood oxygenation without supplemental oxygen, the use of principal drugs that are reversible by antagonists, and the slow and gradual induction of sedation. ${ }^{21,30}$ Pain relief is obtained by successive undersized doses of narcotics, loaded slowly early in the surgery. Narcotics additionally lessen the epinephrine induced hypertension and tachycardia, and it is possible that they also moderate a clinically observed central nervous system activation by epinephrine. By the end of surgery, the narcotics had been metabolized to a great extent, lessening postoperative nausea and urinary retention. Lidocaine however, continues to provide analgesia for several hours post-surgery and patients remain comfortable. ${ }^{31}$ Benzodiazepines in small repetitive doses are used in combination with the narcotics throughout the surgery to provide sedation, dissociation and amnesia and reduce post-operative anxiety. The reliance on narcotics and diazepines that can be reversed with Flumazenil and Naloxone has enhanced safety in the studies cited. ${ }^{21,22}$ Propofol, which is not 
reversible and may be longer acting in some patients, was used to maintain a steady level of sedation and in the dosages used, did not prevent the reversal of narcotics and benzodiazepines.

Furthermore, gradually sedating patients prevents patients from suddenly crashing and allows familiarity with their idiosyncratic responses to the various medications used.

\section{Operative techniques}

The choice of an operative technique impacts the potential risk for DVT. Aggressive panniculus resections necessitate post-operative flexed-postures to protect flap viability. Tight diastasis repairs under general anesthesia likewise increase intraabdominal pressure obstructing venous return. ${ }^{32-37}$ Extensive and/or combined surgeries, especially ones involving circumferential abdominoplasties and intrusions into body cavities take longer and increase the risk for DVT/PE. Longer more extensive and complex surgeries such as circumferential abdominoplasties/body lifts also amplify other surgical risks and raise a question, to what extent do preexisting conditions such as morbid obesity contribute to the DVT risks of these surgeries? and should these cases regardless of the extent of surgery be also classified as -massive weight loss cases?

Finally, it is not known if prolonged pressure as in the pressure caused by lying in one position for a long time and ill-fitting garments (or the pressure of a plaster cast) causes compression or injury to susceptible vessels which may contribute to clotting.

\section{Conclusion}

Abdominoplasties increase venous stasis and therefore the risk of DVT/PE. General anesthesia is likewise an independent risk for DVT. ${ }^{38-40}$ Local anesthesia with sedation has so far shown a reduction in DVT risk without a concomitant increase in anesthesia complications.

Abdominoplasty under sedation imparts a sense of security to patients due to its record of safety. Nonetheless, it requires an experienced and knowledgeable anesthesia provider, takes longer to perform and a supportive staff is essential. Accordingly, clinical judgement ought to be exercised in finding the approach most suitable to the circumstances. 


\section{References}

1. Starling J, Thosani MK, Coldiron BM. Determining the Safety of Office-Based Surgery: What 10 Years of Florida Data and 6 Years of Alabama Data Reveal. Dermatologic Surgery. 2012; 38:2:171-177.

2. Newman, L. Combination Cosmetic Surgeries, General Anesthesia Drive AEs. Medscape Medical News @ 2012 WebMD, LLC. February 9, 2012.

3. Hanke CW. Commentary: Advocating for Mandatory Adverse Event Reporting. Dermatologic Surgery. 2012; 38:2:178-179.

4. Grazer FM, Goldwyn RM. Abdominoplasty assessed by survey, with emphasis on complications. Plast. Reconstr. Surg. 1977.; 59: 513.

5. Teimourian B, Rogers W B. A national survey of complications associated with suction lipectomy: A comparative study. Plast. Reconstr. Surg. 1989; 84: 628.

6. Motura, A.A. Local Anesthesia for Abdominoplasty, Liposuction, and Combined Operations. Aesth Plast Surg. 17:117-124,1993.

7. Nguyen TT, Kim KA, Young RB. Tumescent Mini Abdominoplasty. Ann Plast Surg. 1997; 38(3):209-212.

8. Pravecek EJ, Worland RG. Tumescent abdominoplasty: full abdominoplasty under local anesthesia with i.v. sedation in an ambulatory surgical facility. Plast Surg Nurs. 1998; 18:1: 38-43.

9. Abramson, D. L. Tumescent abdominoplasty: An ambulatory office procedure. Aesthetic Plast. Surg. 1998; 22: 404 .

10. Shestak K.C. Marriage Abdominoplasty Expands the Mini-Abdominoplasty Concept. Plast. Reconstr. Surg. 1999; 103:1020-103.

11. Brauman D. Lipoplasty: A Case for a Low-volume Procedure. Aesthet Surg J. 2000; 20 (5): 373-379.

12. Kryger ZB, Fine NA, and Mustoe TA. The Outcome of Abdominoplasty Performed under Conscious Sedation: Six-Year Experience in 153 Consecutive Cases. Plast. Reconstr. Surg. 2004; 113: 1807.

13. Hatef DA, Kenkel JM, Nguyen MQ, et al. Thromboembolic risk assessment and the efficacy of enoxaparin prophylaxis in excisional body contouring surgery. Plast Reconstr Surg. 2008; 122:269-279.

14. Hatef DA, Trussler AP, Kenkel JM. Procedural risk for venous thromboembolism in abdominal contouring surgery: A systematic review of the literature. Plast Reconstr Surg. 2010;125:352-362.

15. Byun MY, Fine NA, Lee JY, Mustoe TA. The clinical outcome of abdominoplasty performed under conscious sedation: Increased use of fentanyl correlated with longer stay in outpatient unit. Plast Reconstr Surg. 1999; 103: 1260-1266. 
16. Rosenberg MH, Palaia DA, Bonanno PC. Abdominoplasty with Procedural Sedation and Analgesia. Annals of Plastic Surgery. 2001; 46: 5: 485-487.

17. Brauman D. Liposuction abdomminoplasty: an evolving concept. Presented at the ASPS/PSEF/ASMS 71st annual scientific meeting November 2-6, 2002. San Antonio, Texas.

18. Brauman D. Liposuction abdominoplasty: An evolving concept. Plast Reconstr Surg. 2003; 112: 1: 288298.

19. Winocour j, Gupta V, Kaoutzanis C, Shi H, Shack RB, Grotting JC, Higdon KK. Venous Thromboembolism in the Cosmetic Patient: Analysis of 129,007 Patients. Aesth. Surg. J. 2017; 37(3): 337-349.

20. Keyes GR. Commentary on: Venous Thromboembolism in the Cosmetic Patient: Analysis of $129,007$. Aesthetic Surgery Journal. 2017; 37(3) 350-352.

21. Brauman D, Capocci J. Liposuction Abdominoplasty: An advanced Body Contouring Technique. Plast Reconstr Surg. 2009; 124: 5: 1685-1695.

22. Brauman D, van der Hulst RWJ, van der Lei B. Liposuction Assisted Abdominoplasty (LAA): An Enhanced Abdominoplasty Technique. Plast Reconstr Surg Glob Open 2018;6:e1940; doi:

10.1097/GOX.0000000000001940; Published online 14 September 2018.

23. Sozer SA, Basaran K, Alim H. Abdominoplasty with circumferential liposuction: a review of 1000 consecutive cases. Plast Recoinstruct Surg 142; 891, 2018.

24. Dini, G.M., Corsi M.C., Gianini F.L., Ferreira, A.L.M. How Safe Is Thromboprophylaxis in Abdominoplasty? Plast. Reconstr. Surg. 2012; 130: 851e.

25. Klabunde RE. Factors promoting venous return. In, Cardiovascular Physiology Concepts, 2nd edition. 2012; Lippincott Williams \& Wilkins.

26. Blatnik JA. Predicting Severe Postoperative Respiratory Complications following Abdominal Wall Reconstruction. Plast Reconstr Surg . 2012; 130: 83.

27. Brauman D. Diastasis Recti: Clinical Anatomy. Plast Reconstr Surg. 2008; 112;5; 1564-1569.

28. Brauman D. Reply, Letter to the Editor. Diastasis Recti: Clinical Anatomy. Plast Reconstr Surg. 2009; 123(6):1885-1886.

29. Brauman D. Reply, Letter to the Editor. Factors That May Affect. Plast. Reconstr. Surg. 2009; 24(1):334335.

30. Keidan I, Gravenstein D, Berkenstadt H, Ziv A, Shavit I, Sidi A. Supplemental Oxygen Compromises the Use of Pulse Oximetry for Detection of Apnea and Hypoventilation During Sedation in Simulated Pediatric Patients. Pediatrics, August 2008, 122: 2. 
31. Klein JA. Local Anesthesia for Liposuction "Secrets" for performing tumescent liposuction without conventional anesthesia. Plastic Surgery Products. January 2006,46.

32. Saggi BH Sugerman HJ Ivatury RR Bloomfield GL. Abdominal compartment syndrome. J Trauma. 1998; 453:597-609.

33. Watson RA Howdieshell TR. Abdominal compartment syndrome. South Med J. 1998; 914:326-332.

34. Talisman R Kaplan B Haik J et al. Measuring alterations in intra-abdominal pressure during abdominoplasty as a predictive value for possible postoperative complications. Aesthet Plast Surg. 2002; 263:189-192.

35. Tercan M Bekerecioglu M Dikensoy $\mathrm{O}$ et al. Effects of abdominoplasty on respiratory functions: a prospective study. Ann Plast Surg. 2002; 496:617-620.

36. Rodrigues MA Nahas FX Gomes HC Ferreira LM. Ventilatory function and intra-abdominal pressure in patients who underwent abdominoplasty with plication of the external oblique aponeurosis. Aesthet Plast Surg. 2013; 375:993-999.

37. Rodrigues MA, Nahas FX, Reis RP, et al. Does Diastasis Width Influence the variation of the IntraAbdominal Pressure After Correction of Rectus Diastasis. Aest. Plast. Surg. 2015; 35(5) 583-588.

38. Stuzin, J. M., Baker, T. J., and Baker, T. M. Deep venous thrombosis and pulmonary embolus after face lift: A study of incidence and prophylaxis (Discussion). Plast. Reconstr. Surg. 107: 1576, 2001.

39. Daane, S. P., and Rockwell, W. B. Analysis of methods for reporting severe and mortal lipoplasty complications. Aesthetic Plast. Surg. 1999; 23: 303.

40. Christman KD. Death following suction lipectomy and abdominoplasty (Letter). Plast. Reconstr. Surg. 1986; 78: 428 . 

Chapter 7.

Liposuction Assisted Abdominoplasty (LAA): An Enhanced Abdominoplasty Technique

Chapter

\section{Liposuction Assisted Abdominoplasty (LAA): an Enhanced Abdominoplasty Technique}

Brauman, van der Hulst and van der Lei

PLASTIC AND RECONSTRUCTIVE SURGERY Global Open

September 2018, Volume 6, Issue 9 - p 1940 



\title{
Chapter 7.
}

\section{Liposuction Assisted Abdominoplasty (LAA): An Enhanced Abdominoplasty Technique.}

Daniel Brauman, MBBS, MD, Rene R.W.J. van der Hulst MD, PhD and Berend van der Lei, MD, Ph.D.

Plastic and Reconstructive Surgery Global Open: September 2018 - Volume 6 - Issue 9 p e1940

\begin{abstract}
Background: Combining liposuction with abdominoplasties was considered risky during the 1980's and 1990's due to reports of increased complications rates and the belief that liposuction posed a danger to flap circulation ${ }^{1-6}$. However, the corresponding author's intraoperative observations at that time, that liposuction preserved all but the smallest blood vessels, negated the prevailing opinions that liposuction increased the risk to flap circulation, and in October 1996, Liposuction Assisted Abdominoplasty (LAA) was first performed. Thereafter, LAA was honed to become a lipoabdominoplasty technique - not merely a combination of liposuction and abdominoplasty, a technique that utilizes liposuction as a dissection tool - Hydro and Lipo-Dissection, to dissect free and separate the abdominal flap from the deep fascia. Enhanced flap excursion could be demonstrated intraoperatively by selectively transecting the skin retaining ligaments and limiting liposuction to the flap's undersurface, created a vascular lining layer rich in anastomosing blood vessels that provided a rich blood supply to the flap, enabling increased flap excursion.
\end{abstract}

Methods: 593 consecutive ambulatory Liposuction Assisted Abdominoplasties with circumferential torso liposuction and other area liposuction are presented and the surgical technique is illustrated and discussed.

Results: There were no serious adverse events, anesthesia complications, hospital transfers, no venous thromboembolism (VTE) or postoperative respiratory complications in the 593 cases. Patient satisfaction was high, and the results compared favorably with abdominoplasty results published in the scientific literature.

Conclusions: Liposuction Assisted Abdominoplasty is a safe and effective abdominoplasty technique. Extensive clinical experience with LAA suggests longer flap excursion and improved perfusion. It routinely incorporates circumferential torso and other areas liposuction and has commonly included buttock fat grafting. 


\section{Introduction}

Abdominoplasties have grown in popularity in the US incentivized by the growth of outpatient surgery and advances in anesthesia care that enabled safer and faster recoveries. The introduction of Liposuction by Illouz revolutionized body contouring and the addition of liposuction of the torso to complement the results of abdominoplasties, naturally followed ${ }^{7}$. Articles on combining liposuction and abdominoplasty were published during the 1990's however the literature at that time was not generally supportive of this combination, advising caution. ${ }^{1-6,8-13}$ In 2001, Saldanha et. al. described their group's experience (since 2000) with a lipoabdominoplasty technique similar to Illouz'z Suction Abdominoplasty (1992). ${ }^{14,15}$ Since then Lipoabdominoplasty has become synonymous with the Saldanha technique.

Liposuction Assisted Abdominoplasty (LAA), (formerly, Liposuction Abdominoplasty), was first performed in October 1996 by detaching an abdominal flap from the deep fascia with liposuction. The experience with the first 43 patients was presented in 2002 and published in 2003. It offered an increased flap excursion and enhanced perfusion. ${ }^{16-18}$ The experience subsequently gained from 337 consecutive cases (including the first 43) was published in 2009 and demonstrated in Live surgery in 2012. This clinically verified the safety, efficacy and advantages of this approach. 256 additional cases were added since 2009, and their results are included in this paper.

\section{Patients and Methods}

This prospective longitudinal ongoing study was undertaken in 1996. 593 abdominoplasty candidates were divided into two groups. Group -1 - 337 patients, reported on in 2009, and Group -2 - 256 patients, added since 2009. (Table 1).

52 of the 593 abdominoplasty patients were massive weight loss patients who lost weight with bypass surgery and/or lifestyle changes. They underwent extended abdominoplasties and body lifts. 31 of the 593 patients underwent concomitant abdominal hernia repairs. All abdominoplasties since 1996 included circumferential torso liposuction and often other areas liposuction. Additional procedures, most commonly fat grafting, were also performed.

$31 \%$ of Group-1 patients underwent liposuction of one additional area in addition to circumferential torso liposuction, $13 \%$, two additional areas. This compares to $29 \%$ of Group-2 patients who underwent liposuction of one additional area, $12.5 \%$ had two additional areas, 
5.5\% three additional areas, one patient had four areas and another one had six additional areas liposuctioned.

Group -1 included 15 massive weight loss patients (4.4\%) and Group-2 included 73 (14.5\%). Finally, $23 \%$ of Group-1 underwent buttock fat grafting compared with 15\% of Group-2.

Surgery was performed in an office surgical suite under local anesthesia with monitored intravenous sedation. The usual length of surgery was 4-4.5 hours. Patients were discharged home with a responsible adult after approximately 2-3 hours in the recovery room. All patients were seen the morning after surgery, they were allowed to walk upright, showered, and returned to normal activity (Figs. 1a, 1b). Strenuous abdominal exercises were avoided for 3 months when diastasis repair was performed.

The corresponding author Daniel Brauman (DB) performs surgery under intravenous sedation. The coauthor Berend van der Lei (BvdL) has been performing this procedure since 2010 under general anesthesia. ${ }^{19,} 20$

Table 1. Data on the 593 patients.

\begin{tabular}{|l|l|l|}
\hline Parameters: & Group 1. 1996-2009 & $\begin{array}{l}\text { Group 2. 2009- } \\
\mathbf{2 0 1 7}\end{array}$ \\
\hline Number of patients: & 337 & 256 \\
\hline $\begin{array}{l}\text { Age (in years): } \\
\text { Average age (in years): }\end{array}$ & $\begin{array}{l}21-72 \\
36\end{array}$ & $\begin{array}{l}17-73 \\
44.1\end{array}$ \\
\hline Gender: & Females: 330 & Females: 254 \\
Weight range: & Males: 7 & Males: 2 \\
Average weight: & $100-307$ lbs. & $\begin{array}{l}115-265 \text { lbs. } \\
168.1 \text { lbs. }\end{array}$ \\
\hline $\begin{array}{l}\text { BMI range: } \\
\text { Average BMI: }\end{array}$ & $\begin{array}{l}143.5-41.7 \\
\text { lbs. }\end{array}$ & $\begin{array}{l}21-42.8 \\
29.4\end{array}$ \\
\hline $\begin{array}{l}\text { Number: } \\
\text { Weight range: }\end{array}$ & $\begin{array}{l}\text { Hanging } \\
\text { panniculus: } 158\end{array}$ & $\begin{array}{l}\text { Hanging } \\
\text { Panniculus: } 174 \\
60-2650 \text { gr. }\end{array}$ \\
Average: & $\begin{array}{l}100-3125 \text { gr. } \\
384 \text { gr }\end{array}$ & 858 gr \\
\hline
\end{tabular}




\begin{tabular}{|c|c|c|}
\hline${ }^{*}$ Total Fat Removed: Average: & $\begin{array}{l}50-7800 \mathrm{ml} . \\
2100 \mathrm{ml} .\end{array}$ & $\begin{array}{l}20-8200 \mathrm{ml} \\
2234 \mathrm{ml} \text {. }\end{array}$ \\
\hline $\begin{array}{l}\text { No. of body areas treated in addition to Circumferential Torso } \\
\text { Liposuction. } \\
\text { No. of patients with additional 1-2 area liposuction }\end{array}$ & 149 patients & 64 patients \\
\hline $\begin{array}{l}\text { No. of patients who underwent procedures in addition to } \\
\text { liposuction }\end{array}$ & 85 patients & 53 patients \\
\hline Fat grafting to buttocks & 38 patients $(15 \%)$ & 59 patients $(23 \%)$ \\
\hline Massive weight loss patients & 15 patients $(4.4 \%)$ & 37 patients (14.5\%) \\
\hline Average follow-up average: & 7 months & 7.3 months \\
\hline Range: & 1 week - 9 years & 4 weeks-20 years \\
\hline
\end{tabular}

The volume of fat recorded at 30 minutes did not significantly change when allowed to settle for an hour or two - In 189 cases. ${ }^{16 *}$ The volume of fat removed was the volume of fat measured after allowing it to settle for 30 minutes, Not the volume of the aspirate. The volume of aspirate typically varies with the volume of infiltrate and with the method of fat removal and its resultant lipolysis - fat breakdown and dispersion.
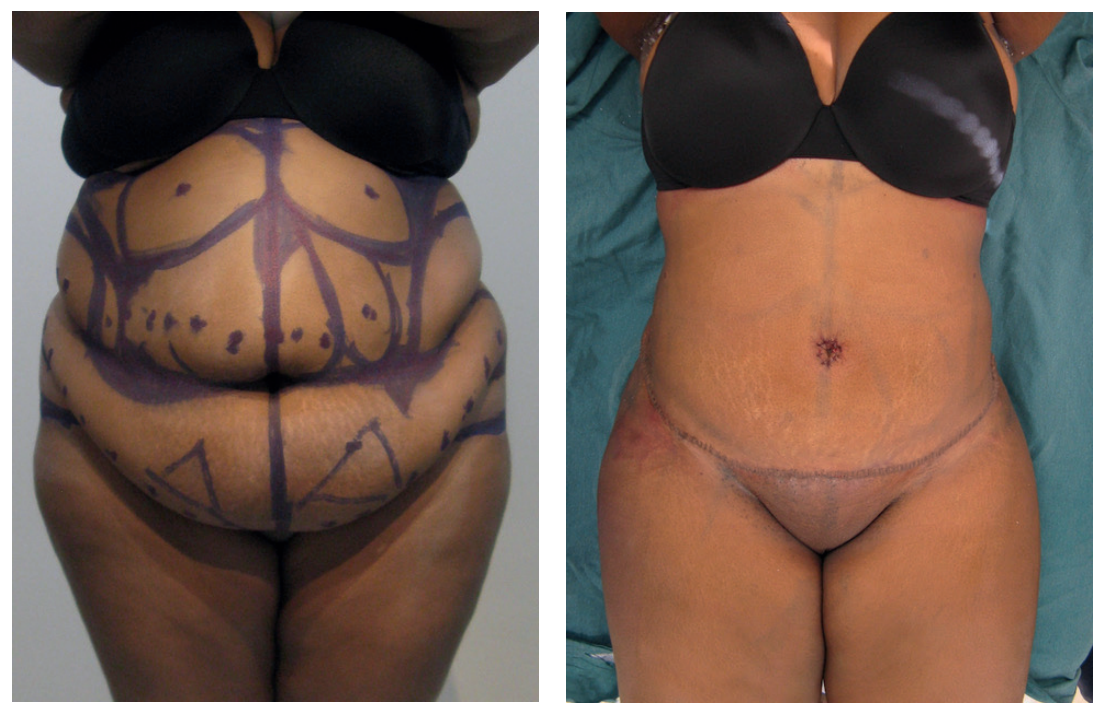

Fig. 1. Before and next morning after LAA with circumferential torso liposuction. Note the patient is fully extended and the abdominal flap at its inferior border is healthy looking. The incision is at the pubic hairline with pubic uplift also performed. 


\section{Surgical Technique}

First, anesthetic fluid of the surgeon's choosing is infiltrated between the deep fascia and the subcutaneous tissue with $3 \mathrm{~mm}$ infiltration cannulas attached to $60 \mathrm{cc}$ Toomey syringes, to separate the subcutaneous tissue from the muscular abdominal wall - Hydro-dissection. The point of entry is from the right or left sides, superior to the umbilicus.

The entry to the surface of the muscular plane is done "by feel", a maneuver not difficult to master. Initially infiltration/separation is performed in the epigastric area and then in the infra umbilical area. The plane of dissection remains on the deep fascia, deep to Scarpa's fascia. The cannula slips under Scarpa's fascia because its point of entry is superior to the umbilicus which is where Scarpa's fascia ends as a discernable layer (Fig.2). Next, Liposuction follows infiltration, removing the deep fat layer bordering the muscle and disrupting the stiff skin retaining ligaments while preserving the flexible blood vessels - Lipo-dissection. The skin retaining ligaments affix the skin to the fascia and form body creases such as the tendinous intersections (six pack). Disrupting - is done by performing liposuction while applying traction to the flap. Traction places the ligaments under tension. The ligaments are stiff and will tear, the blood vessels however are flexible and will move aside (LAA Video 1). Disruption of the skin retaining ligaments is sufficient to provide flap mobility when the patient's skin and subcutaneous tissue is loose. Otherwise, discontinuous undermining is necessary, and some neurovascular perforators may need to be divided/sacrificed.

Detaching the subcutaneous tissue from the muscle with liposuction and removing the fat bordering the muscle creates a "safe zone", a space that prevents inadvertent perforations. Scarpa's fascia's superficial surface is next separated from the infraumbilical skin by infiltrating and liposuctioning the subcutaneous fat. This exposes Scarpa's fascia and preserves femoral artery branches coursing on the fascia's superficial surface (Fig. 3 ). Further liposuction of the flap's undersurface is used to detach, thin and even the flap. The flap ends up being lined by a compact layer of fibrous tissue (scaffolding) containing blood vessels, nerves and lymphatics coined - Supporting tissue layer. The Supporting tissue distributes the blood supply and forms a distinct compact deep border for the flap (igs. 4-6).

Next, a curvilinear incision is made, Scarpa's fascia is split in the midline to preserve its vessels and lymphatics, the abdominal flap is elevated sharply and bluntly from the deep fascia, preserving the Supporting Tissue layers on the undersurface of the flap, the abdominal wall and the deep and superficial surfaces of Scarpa's fascia. Traction on the flap is applied to evaluate the desired tension and neurovascular perforators preventing flap downward movement are divided. 


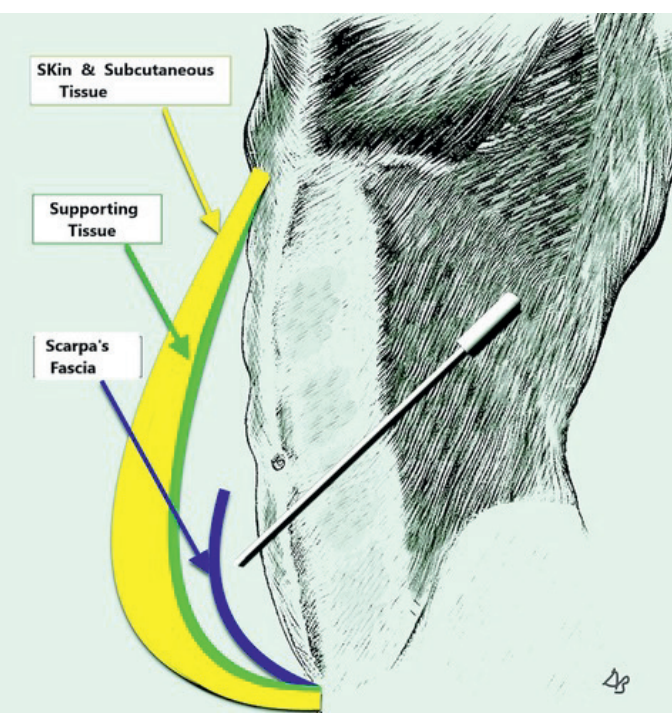

Fig. 2. Depicting the cannula's point of entry from the side, superior to the umbilicus. The cannula's tip is deep to Scarpa's fascia. Supporting tissue (in green) forms the deep layer of the abdomina flap. Supporting tissue (not shown) is also present on the deep fascia and on Scarpa's fascia's deep and superficial surfaces.

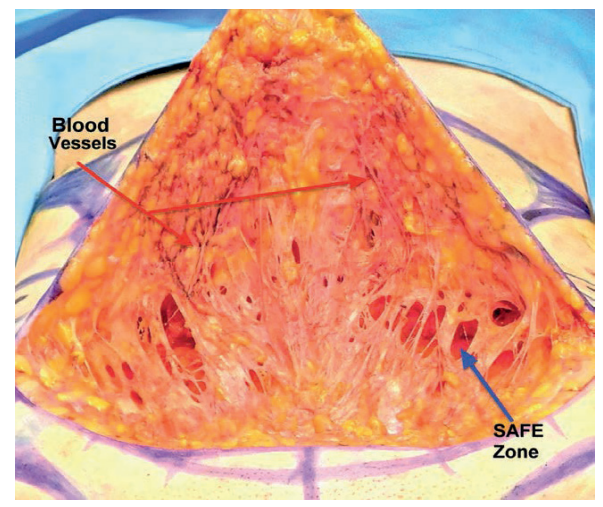

Fig. 3. The superficial surface of Scarpa's fascia had been separated from the skin and subcutaneous tissue with liposuction. The blue arrow points to the "safe zone," a space created by liposuction deep to Scarpa's that separated the deep surface of the fascia from the

muscle. The red arrows point to vaso-constricted femoral artery branches coursing on the fascia toward the skin. These blood vessels can augment the abdominal flap's blood supply.

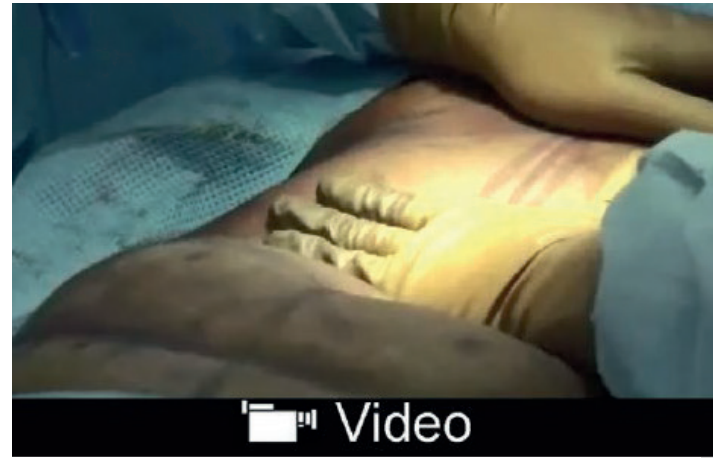

Video Graphic 1. See video, Supplemental Digital Content 1, which displays lipo-dissection and lipo-disruption of ligaments. This video is available in the "Related Videos" section of the Full-Text article at PRSGlobalOpen.com or at http://links.Iww.com/PRSGO/A856.

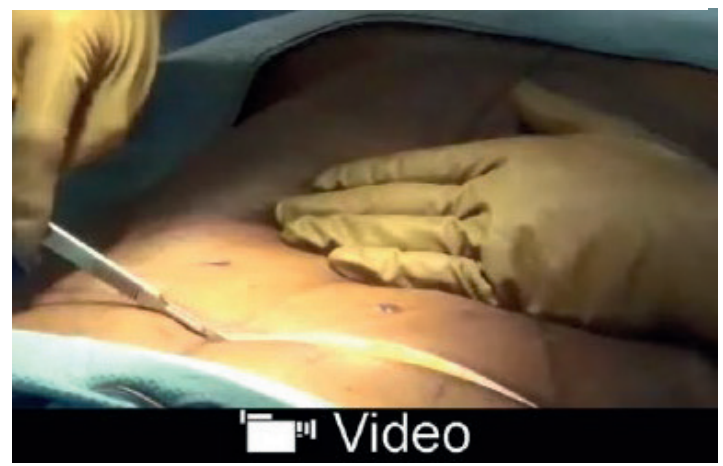

Video Graphic 2. See video, Supplemental Digital Content 2, which displays extension of flap excursion by targeted ligament and perforator release This video is available in the "Related Videos" section of the FullText article at PRSGlobalOpen.com or at http://links.Iww.com/PRSGO/A857.

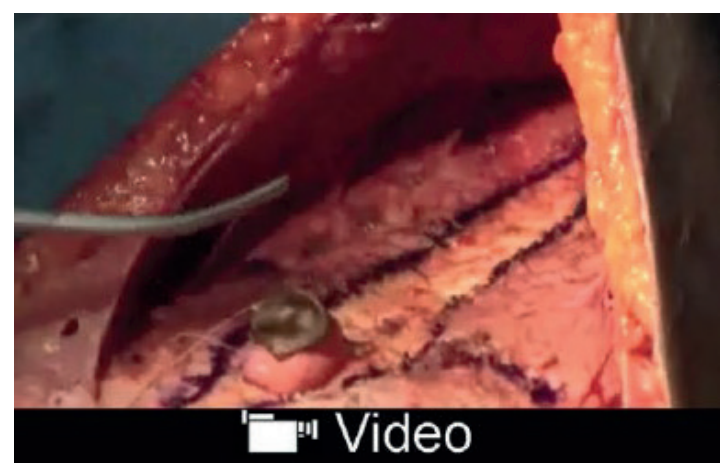

Video Graphic 3. See video, Supplemental Digital Content 1, which displays supporting tissue layer and segmental diastasis repair. This video is available in the "Related Videos" section of the Full-Text article at

PRSGlobalOpen.com or at http://links.Iww.com/PRSGO/A858. 
Panniculus excision is done last. The operating table is extended (not flexed), and the flap is placed under tension while the panniculus is excised. Panniculus excision is done by superficial liposuction just distal to the excision line that exposes a strip of intact Supporting tissue which is preserved distal to the flap's edge. Bleeding from the edge is expected with the flap under tension and the patient fully extended (LAA Video 2). Segmental Diastasis repair is performed when indicated and closure of the lower abdominal incision is done with tissue overlap to prevent linear scar contracture (LAA Video 3). ${ }^{21-23}$ Drains may be used but weekly tapping is an alternative depending on surgeon's choice. Tacking sutures are not recommended for fear of damage to the flap's vascular Supporting Tissue layer. Patients are then positioned on their side and circumferential torso and other areas liposuction is carried out.
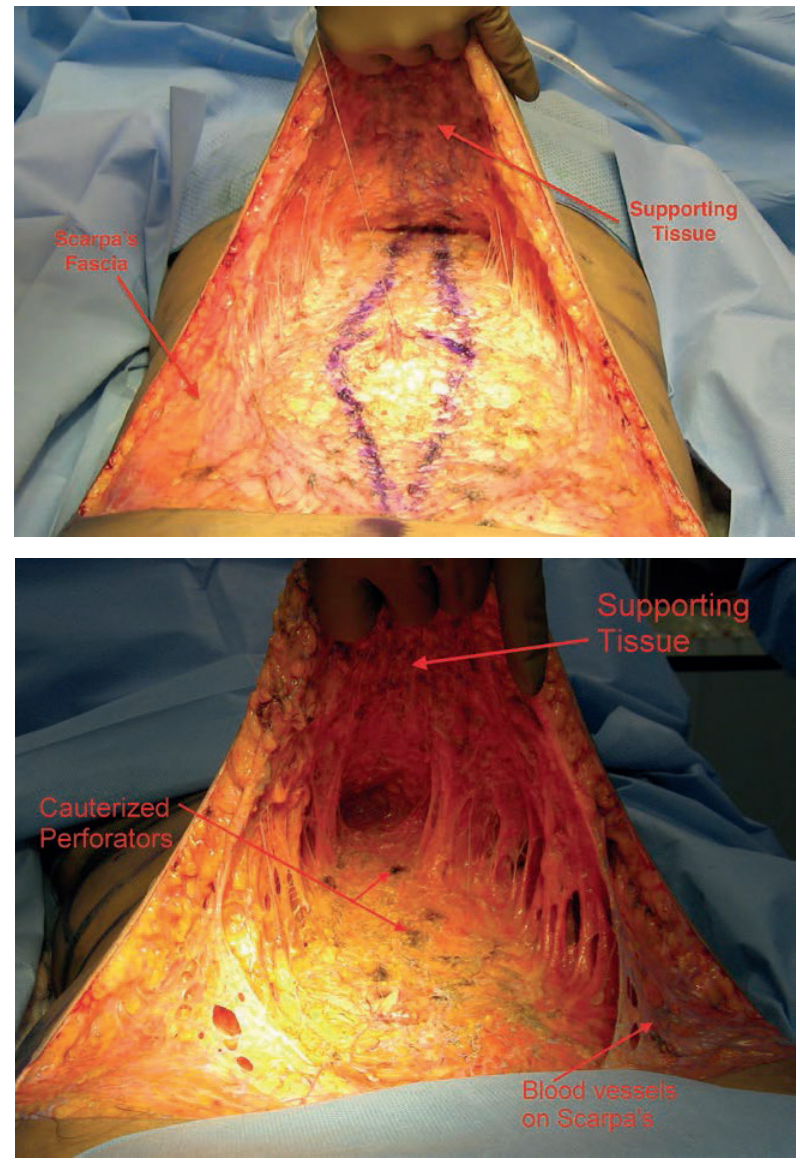

Fig. 4. The flap is even, lined by a supporting tissue layer that is "bluish" in color as epinephrine vaso-constriction is abating, and veins are filling up. Scarpa's, split in the midline, its vessels still vaso-constricted, nevertheless, bleeding from the distal edge was observed. In this patient, tissues were tight, and perforators needed to be transected to obtain more mobility. The flap can withstand loss of some important perforators since it is perfused through its supporting tissue vessels.

Fig. 5. The tissue was looser and less perforators needed to be sacrificed. Note femoral artery branches on Scarpa. 


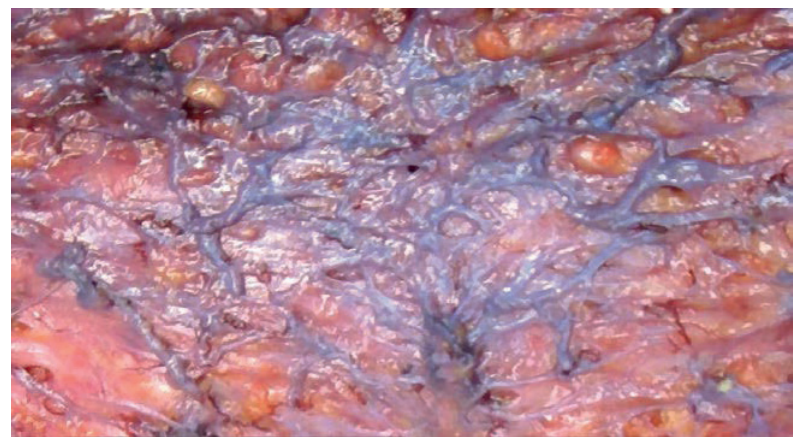

Fig. 6. A magnified section of a supporting tissue layer. Epinephrine vaso-constriction is subsiding with veins becoming filled with blood, first.

\section{Results}

593 consecutive patients who underwent office-based Liposuction Assisted Abdominoplasty (LAA) under local anesthesia with monitored intravenous sedation between 1996 and 2017 are included. No abdominoplasty patients were excluded unless medically unsuitable for surgery. Overall, there were no serious complications, venous thromboembolism (VTE) or unplanned hospital transfers in the 593 cases. Patient satisfaction was high as evidenced by satisfaction surveys and the many referrals by satisfied patients over 21 years.

Group-1- 337 patients operated on between 1996 - 2009 suffered no anesthetic complications or serious surgical complications. There were five (1.4\%) late home acquired infections before 2005, attributable to the prolonged use of drains and six cases $(1.7 \%)$ of minor tissue necrosis in smokers that healed without affecting the final aesthetic results. 2 of the 6 cases of minor necrosis involved $T$ junctions and two were caused by drain-site pressure. ${ }^{16-19,}{ }^{21}$ Drains were discontinued in 2005 but reinstated several years later because of the increase in seroma incidence caused by the inclusion of many more massive weight loss patients.

In Group-2 - 254 patients, 2009-2017, there were also no serious complications, VTE, anesthesia complications or unplanned hospital transfers. One patient, placed on Enoxaparin Sodium prophylaxis the morning after surgery was found to have bled under the flap, a week later. She was treated with aspirations during the next few visits without any aesthetic long-term sequelae. There was $1(0.39 \%)$ home acquired infection of a seroma in a chronic rheumatoid arthritis, massive weight loss patient who was difficult to care for, repeatedly neglecting to close and compress her drain's suction bulb, admitting herself to the hospital to qualify for home care and trying this again after discharge but being refused admission to another hospital. There were no other cases of infection and no marginal necrosis. 
Seroma formation occurred much more commonly in massive weight loss patients. Groups-2 had 37 massive weight loss patients and seroma formation affected 7 of them (19\%). In comparison, the remaining 219 non-massive weight loss patients in that group, sustained only 3 seromas (1.3\%). In Group-1, 5 of 15 massive weight loss patients had seromas (33\%) and no seromas occurred in the remaining 322 patients of that group. Seromas contributed to excess fibrosis and scar contractures. ${ }^{24}$ In Group-2, there were 2 (5\%) scar revisions in the 37 massive weight loss patients compared to $4(1.3 \%)$ revisions in the remaining 219 cases. Lastly, of the 6 scar revisions in Group-2, 3 were due to seromas (50\%).

Group-1 seromas were treated successfully with 4-5 mg Kenalog ${ }^{\circledR}-10$ Injections into the seromas (triamcinolone acetonide injectable suspension, USP, Bristol-Myers Squibb Company. Princeton, NJ, USA). In Group-2, the 7 seromas were treated with dehydrated alcohol-sclerotherapy. Furthermore, the 37 massive weight loss patients who incurred 7 seromas, resulted in the reinstatement of drains. These numbers confirmed other published data that massive weight loss patients are prone to seromas and their resultant scar contractures. ${ }^{24}$ Sclerotherapy with dehydrated alcohol continues to be performed by an interventional radiologist, interested in seroma treatment at the affiliated hospital. The procedural risks in the 593 are summarized in (table 2).

Table 2. Procedural risks- 593 patients. 1996-2017. Groups 1 \& 2

\begin{tabular}{|l|l|l|l|}
\hline & $\begin{array}{l}\text { Group -1 } \\
\mathbf{3 3 7} \text { patients }\end{array}$ & $\begin{array}{l}\text { Group -2 } \\
\mathbf{2 5 4} \text { patients }\end{array}$ & $\begin{array}{l}\text { Total } \\
\mathbf{5 9 1} \text { patients }\end{array}$ \\
\hline Seromas & $5(1.4 \%)$ & $7(2.7 \%)$ & $12 \%)$ \\
\hline Infections & $\begin{array}{l}5(1.4 \%) \text { Late, Home } \\
\text { acquired. }\end{array}$ & $\begin{array}{l}1(0.39 \%) \text { “difficult" } \\
\text { patient }\end{array}$ & $6(1 \%)$ \\
\hline Hematomas & 0 & $\begin{array}{l}1 \text { (0.4\%). Due to } \\
\text { Enoxaparin }\end{array}$ & $1(0.16 \%)$ \\
\hline
\end{tabular}

Abdominal scar contractures can occur despite the interposition of subcutaneous tissue under the flap. The successful release of 6 contractures undertaken a year after the surgery consisted of excising contracted scars and once again interposing subcutaneous tissue under the incision. Currently, short courses of oral steroids are being evaluated in susceptible individuals to prevent contracture. Pfizer ${ }^{\circledR}$ Medrol $^{\circledR}$ Dosepak $^{\mathrm{TM}}$ (Methylprednisolone) $4 \mathrm{mg}$. The experience gained from this large series of patients helped anticipate scar contractures and seromas. Patients that appear prone to scar contractures are offered alternative procedures such as liposuction. Liposuction 
can harness patients' tendency to contract their scars and utilize it to their advantage by contracting and eliminating a hanging panniculus (Figs. 7a, 7b).
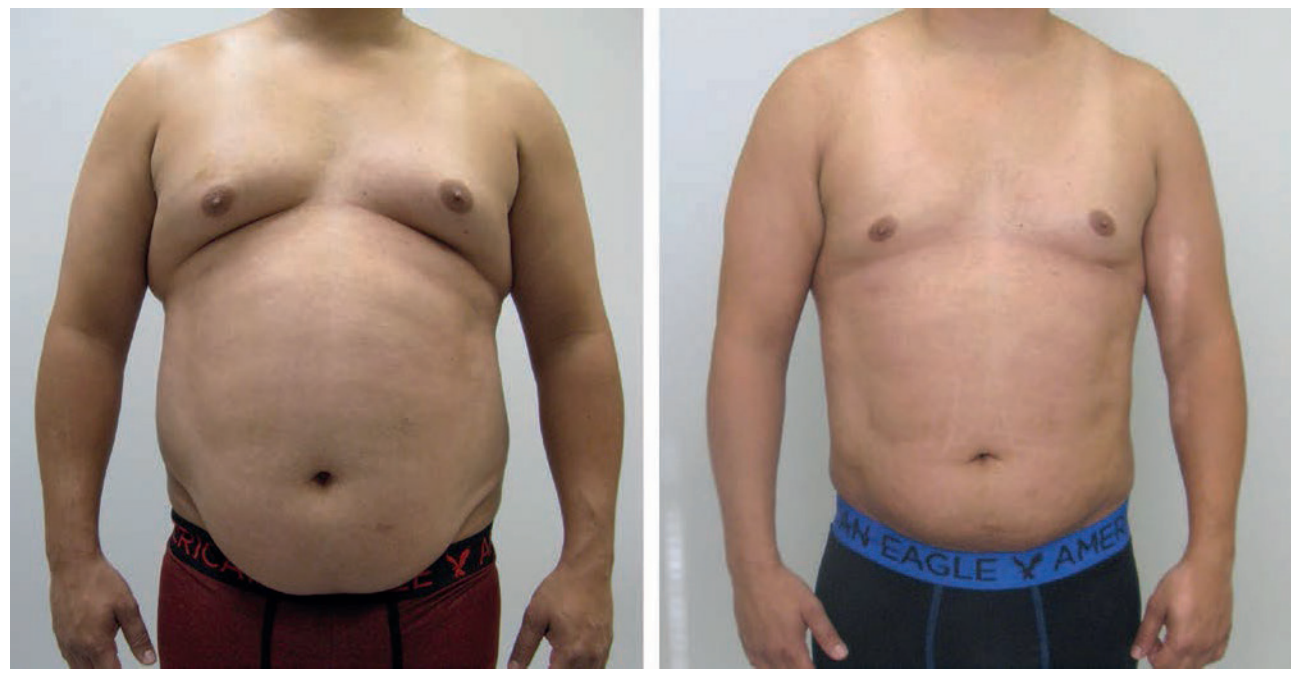

Fig. 7. Before and 6 weeks after liposuction of abdomen chest and circumferential torso. Note the improvement in the hanging panniculus and the smooth result possible when anatomical liposuction separates the muscular abdominal wall from the panniculus enabling precise anatomic liposuction.

Concomitant abdominal hernia repair without mesh was done in 31 of the 593 abdominoplasties (Group-I and II). Follow-up data showed no recurrence in 18 patients who returned for followup (3.5 months -6 years, average, 20.2 months).

The majority of patients reported experiencing a marked improvement in their appearance and function. Very few, mostly in the massive weight loss group were hard to please and presented body image issues in addition to an increase frequency of seromas and scar contractures. The added experience with the technique and the newly available urine nicotine test after 2009, resulted in the absence of flap ischemia and any marginal necrosis. Patients who smoked were not treated. Overall, patient satisfaction continued to remain very high, evidenced by satisfaction surveys and referrals. Patients were pleased with aesthetic results that were enabled by the safe inclusion of routine circumferential torso liposuction, as well as additional area liposuction that integrated their abdominal contouring with their figure and often the ability to conceal their scars (Figs. 8, 9). 

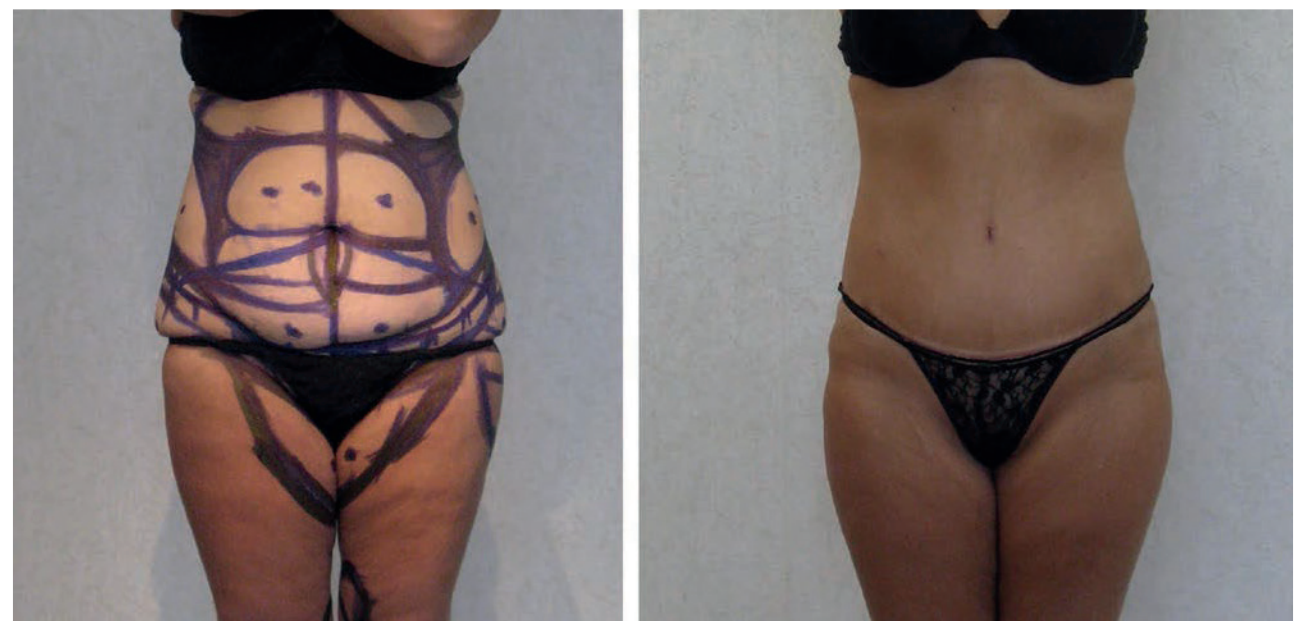

Fig. 8. Before and after photographs of LAA with circumferential torso and thighs liposuction. This patient healed with a slightly hypertrophied scar visible just above the scant underwear but able to be hidden by it. A smooth transition to the pubic area is present. A scar contracture would have created a secondary fold negating the smooth transition, and the patient would not have been able to conceal her scar with underwear.

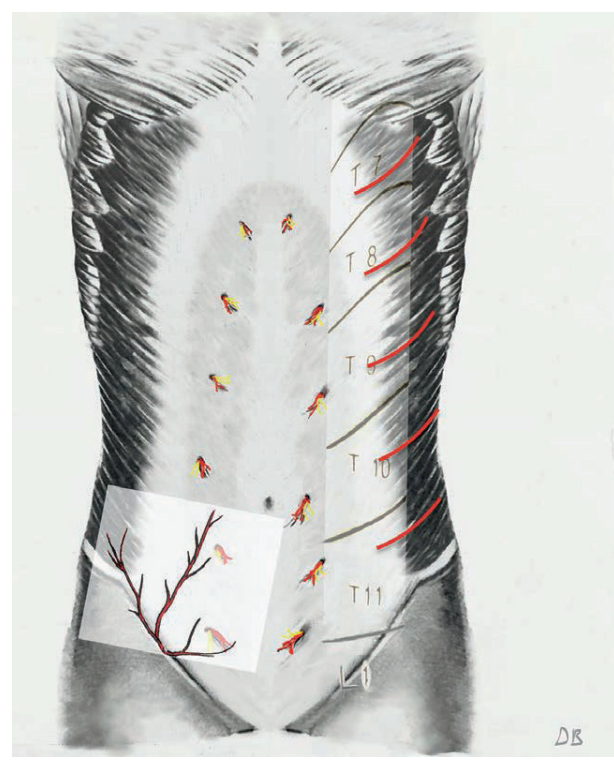

Fig. 9. A simplified view of the abdominal wall's blood supply. On the right, the deep anterior and lateral neurovascular perforators- T7- L1. On the left, the 3 superficial branches of the femoral coursing on Scarpa's fascia.
For the 43 patients who underwent liposuction abdominoplasties beginning in 1997 (group III), abdominal incisions were closed with a fluidtight, absorbable, running subcuticular suture. However, after one minor postoperative wound seroma (in 1999), an interrupted staple closure was adopted in all cases. After their operations, two patients had minor revisions to their neoumbilicus, two others underwent additional (preplanned, staged) liposuction procedures, and one underwent a mastopexy. Most importantly, there were no occurrences of deep venous thrombosis, flap necrosis, sensory loss, or other complications.

Before the operation, the intraabdominal fat content (volume) was evaluated in all 60 patients (groups I, II, and III). The volume was then classified as small, medium, or large. (The author routinely uses this classification when deciding the appropriateness of a rectus sheath plication for a given patient.) In every case, the postoperative appearance of the abdomen correlated directly with the preoperative evaluation of the intraabdominal fat content, regardless 
of whether a diastasis recti repair was performed (23 of 60 patients). More specifically, 25 patients whose intraabdominal fat content was judged as small recovered with a flat abdomen. Eighteen patients estimated to have medium fat volume healed with a medium protrusion, and 17 patients assessed with a large volume ended up with a larger protrusion.

Data were also available for 11 of the 23 patients (groups I, II, and III) in whom diastasis repair was performed. At an average follow-up time of 29 months from the time of diastasis repair, seven patients experienced recurrence of their diastasis when the intraabdominal fat volume was evaluated as large (three patients) or medium (four patients). Four diastasis repair patients whose intraabdominal fat was evaluated as small did not experience a recurrence.

\section{Discussion}

The fundamental requirement for a safe and effective abdominoplasty is a well-perfused and mobile flap. Flap mobility, in addition to umbilical release, requires the detachment of the skin retaining ligaments that affix the skin to the fascia (especially) at the tendinous intersections (six pack) and along the midline (Linea Alba) and flap perfusion is maximal when it contains a wellperfused vascular network.

Liposuction Assisted Abdominoplasty (LAA) evolved gradually. In 1996, it was named Liposuction Skin Excision. ${ }^{16,} 25$ Liposuction loosened the subcutaneous tissue, the skin was stretched, and umbilical transposition was performed. Yet, this only allowed limited flap mobility. However, Intra-operative observations revealed that placing the flap under tension while performing liposuction, disrupted - tore, the stiff, unyielding skin retaining ligaments and spared the (more flexible) blood vessels resulting in greater flap mobility than was possible with liposuction alone. (LAA Video 1)

To further increase flap mobility, discontinuous dissection and selective transection of the unyielding retaining ligaments and freeing the more stretchable neurovascular perforators was performed. The flap was freed sharply and bluntly to the inframammary crease superiorly and to the flanks laterally where the surgical dissection blended with the circumferential torso liposuction that further released the flaps lateral borders. In order to maximize flap mobility some periumbilical perforators could be sacrificed without a discernible effect on the flap's profuse blood supply (LAA Video 2).

To clinically confirm the extended downward excursion of the LAA flap compared to the Saldanha flap was done by performing liposuction only, following it with tension on the liposuctioned flap, to disrupt the ligaments and then carrying out a discontinuous dissection to further mobilize the flap (LAA Video 2). 
Further intraoperative observations revealed that a well-defined vascular layer ended up lining the flap's undersurface. Liposuction (with blunt tip cannulas - not laser or ultrasound), limited to the flap's deep surface, was observed to have "plucked" fat globules from the connective tissue scaffolding while leaving the loose scaffolding and its vessels and nerves largely intact. The scaffolding and its content - vessels, nerves and lymphatics appeared to have collapsed and formed a compact connective tissue layer that was coined the Supporting tissue layer, and when the epinephrine vasoconstriction subsided, the undersurface layer was found to consist of a rich network of arteries, nerves and lymphatics, embedded in a fibrous-connective tissue scaffolding (Figs. 4-6).

The resultant flap ended being an island flap based on neurovascular perforators lined by a compact vascular Supporting tissue layer rather than a friable adipose tissue undersurface that could have contained de-vascularized fat fragments. Furthermore, the flap could be further thinned and evened by open liposuction of its undersurface (LAA video 1). Not performing liposuction throughout the full thickness of the subcutaneous tissue in contrast to the Saldanha approach of liposuctioning the entire subcutaneous tissue (except for the "subcutaneous $1 \mathrm{~cm}$ "), seemed safer, as no intrusion into the flap was judged best. ${ }^{15}$

The flap's blood supply is distributed through the anastomosing network of the Supporting tissue undersurface supplied by deep T-7-L-1 lateral and anterior cutaneous neurovascular perforators. Additional blood supply may be harnessed by incorporating the 3 superficial branches of the femoral artery coursing on the superficial surface of Scarpa's fascia. Abdominoplasty techniques resecting the infra-umbilical panniculus also resect Scarpa's fascia and its vessels, LAA, can preserve some of these vessels as Scarpa's Fascia's superficial surface is separated with liposuction and split in the midline. (LAA Video 2). These additional vessels, when present, (unless damaged by $\mathrm{C}$-sections etc.), supercharge the flap. ${ }^{26-33}$

A recent article by Smith, Sr. \& Jr distinguishes between lipoabdominoplasty techniques such as Saldanha and Brauman and analyzes the abdominal wall's blood supply He concludes that If perforators of the epigastric system vessels are spared, liposuction should be able to be safely performed on the central abdominal skin, Huger zone-l. ${ }^{34}$ Smith also surmises that Brauman likely preserves these perforators. ${ }^{18,19}$ This may very well be so in many cases, however, when the flap is tight, epigastric central perforators are sacrificed in favor of umbilical central perforators that are usually longer, larger and more flexible since Brauman relies heavily on the flap's anastomosing-vascular Supporting tissue lining for the distribution of blood to the central, vulnerable portion of the flap (LAA Video 2).

Compared with the Saldanha's flap, the LAA's flap improved perfusion and excursion can be demonstrated intraoperatively as the flap is released and extended and when the panniculus is 
excised with the operating table extended and the flap under tension. (LAA Videos 2 \& 4). The well perfused flap can be advanced lower, and its safe and longer excursion is attested to by the absence of marginal necroses in several hundred consecutive abdominoplasties, the surgical results obtained even in the presence of a high umbilicus and limited epigastric laxity (Figs 10a \& b) and the ability of patients to walk upright to the recovery room after surgery. Allowing patients to walk upright, likely also helps with respiration and venous return. Additionally, flap sensibility appears to be much enhanced and infection is rare because of the flap's abundant perfusion.

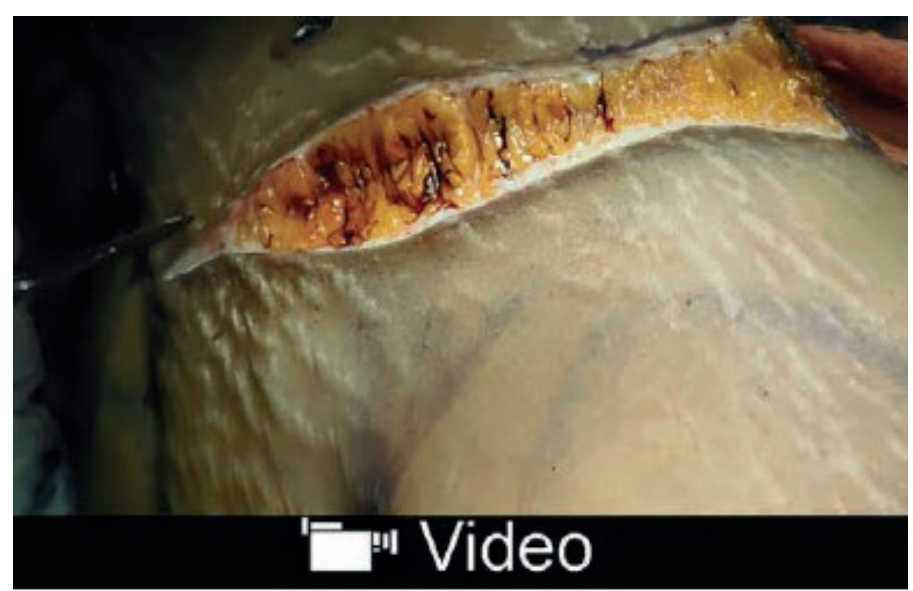

Video Graphic 4. See video, Supplemental Digital Content 4, which displays excision of panniculus with liposuction and bleeding from the edge with flap under tension, http://links.lww.com/PRSGO/A859.
Seroma formation remains a persistent problem in abdominoplasty. ${ }^{35,} \quad 36$ Intraoperatively, massive weight loss patients appear to exhibit denser panniculi with an abundance of fibrous tissue and enlarged blood vessels. It seems that weight loss results in the loss of fat but the Supporting tissue, containing enlarged, numerous blood vessels, nerves and lymphatics, appears to have been retained. This could explain the preponderance for seroma formation and the potential for lengthier surgeries and blood loss in massive weight loss surgery.

Preservation of the supportive tissue layer on the superficial surface of Scarpa's fascia (that may contain lymphatics), is probably beneficial for seroma prevention. The recent finding that the lymphatic drainage from the lower abdomen courses superficial to Scarpa's and pierces the fascia low, 2-3 cm proximal to the inguinal ligament supports this assumption. ${ }^{37}$

Seromas have been effectively treated by the corresponding author with local and systemic steroids prior to 2009, and Janis et. al have recently confirmed the inflammatory nature of seroma fluid. ${ }^{19,} 38$ Treatment with steroids may be feasible for long standing seromas however when steroids are used for prevention or treatment of fresh seromas the benefits have to be weighed against the potential effect of steroids on the tensile strength of the healing tissues. 

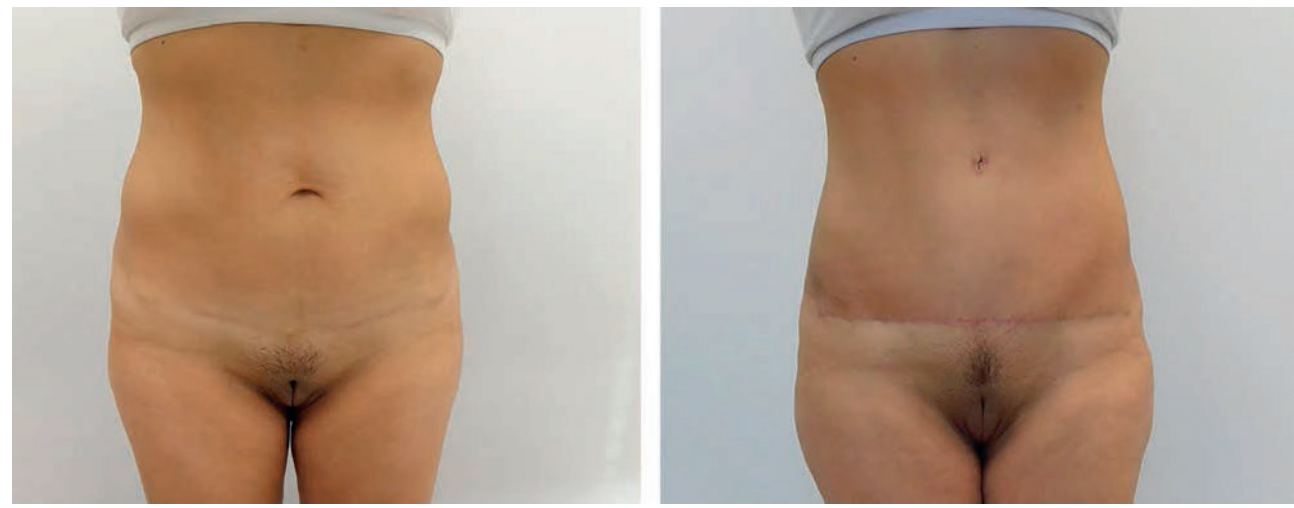

Fig. 10. Thin patients with tight epigastric tissues/skin and high umbilicus, demonstrating the extent of the safe downward reach of the flap due to its superior mobility and rich blood supply distributed by its supporting tissue layer.

\section{Conclusion}

The LAA technique is based on sound surgical principals. The LAA flap's extended downward reach is enabled by its rich blood supply. The $L A A$ technique is uncomplicated to perform. The basic surgical maneuvers of Hydro and Lipo-dissection and separation of the subcutaneous tissue from the muscular fascia are straightforward and the rest follows from there. Backed by a 22year experience gained by the authors and others, the LAA technique is suggested to surgeons as a proven alternative to other lipoabdominoplasty techniques.

\section{References}

1. Christman KD. Death following suction lipectomy and abdominoplasty (Letter). Plast. Reconstr. Surg. 1986; 78: 428.

2. Abbes $M$ and Bourgeon $Y$. Fat embolism after dermatolipectomy and liposuction (Letter). Plast Reconstr Surg. 1989; 84: 546.

3. Ersek RA. Serial suction lipectomy. Clin Plast Surg. 1989; 16: 313.

4. Dellerud E. Abdominoplasty Combined with suction lipoplasty: a study of complication, revisions, and risk factors in 487 cases. Ann Plast Surg. 1990; 25:333-338.

5. Lockwood T. High-lateral-tension abdominoplasty with superficial fascial system suspension. Plast. Reconst. Surg. 1995; 96: 3 :603-615.

6. Matarasso, A. Liposuction as an adjunct to full abdominoplasty. Plast. Reconstr. Surg. 2000; 106: 5: 1197. 
7. Illouz YG. Body contouring by lipolysis: a 5-year experience with over 3000 cases. Plast Reconstr Surg. 1983; 72: 591-597.

8. Nguyen, T. T., Kim, K. A., and Young, R. B. Tumescent mini abdominoplasty. Ann. Plast. Surg. 1997; 38: 209.

9. Cardenas-Camarena, L., and Gonzalez, L. E. Large-volume liposuction and extensive abdominoplasty: A feasible alternative for improving body shape. Plast. Reconstr. Surg. 1998; 102: 1698.

10. Baroudi, R. Liposuction as an adjunct to a full abdominoplasty revisited (Discussion). Plast. Reconstr. Surg. 2000; 106: 1203.

11. Shestak, K. C. Liposuction as an adjunct to a full abdominoplasty revisited (Discussion). Plast. Reconstr. Surg. 2000; 106: 1205.

12. Heppe, H. P. Combined liposuction with abdominoplasty (Letter). Plast. Reconstr. Surg. 2001; $108: 577$.

13. Avelar, J. M. Abdominoplasty without panniculus undermining and resection: Analysis and 3-year follow-up of 97 consecutive cases. Aesthetic Surg. 2002: J. 22: 16.

14. Illouz YG. A new safe and aesthetic approach to suction abdominoplasty. Aesthetic Plast Surg. 1992; $16: 3$.

15. Saldanha OR, Pinto EB, Matos WN Jr, Lucon RL, Magalhães F, Bello EM. Lipoabdominoplasty without undermining Aesthet Surg J. 2001; 21:518-526.

16. Brauman D. Lipoplasty: A Case for a Low-volume Procedure. Aesth Surg J. 2000; 20 (5): 373-379.

17. Brauman D. Liposuction Abdomminoplasty: An evolving concept. Presented at the ASPS/PSEF/ASMS $71^{\text {st }}$ annual scientific meeting November 2-6, 2002. San Antonio, Texas.

18. Brauman D. Liposuction abdominoplasty: An evolving concept. Plast Reconstr Surg. 2003; 112: 1: 288298.

19. Brauman D, Capocci J. Liposuction Abdominoplasty: An advanced Body Contouring Technique. Plast Reconstr Surg. 2009; 124(5):1685-1695. Brauman D. Anatomical Liposuction \& Liposuction Abdominoplasty. The second Huffstadt International Conference in Groningen, The Netherlands. University Medical Centre of Groningen. November 12-13, 2010.

20. Brauman D. Anatomical Liposuction \& Liposuction Abdominoplasty: Live Surgery. Sint Lucas Andreas Hospital, Amsterdam, The Netherlands. NVEPC 6th International Live Surgery and Congress on Aesthetic Plastic Surgery Body contouring, Amsterdam, The Netherlands. December 7 \& 8, 2012.

21. Brauman D. Diastasis Recti: Clinical Anatomy. Plast Reconstr Surg. 2008; 112;5; 1564-1569. 
22. Brauman D. Reply, Letter to the Editor. Diastasis Recti: Clinical Anatomy. Plast Reconstr Surg. 2009; 123(6):1885-1886.

23. Brauman D. Reply, Letter to the Editor. Factors That May Affect. Plast Reconstr Surg. 2009; 124(1):33433.

24. Di Summa PG, Wettstein R, Erba P, Raffoul W, Kalbermatten DF. Scar asymmetry after abdominoplasty: the unexpected role of seroma. Ann Plast Surg. 2013 Nov; 71(5):461-3.

25. Pitman GH. Commentary. Aesthetic Surgery Journal 2000; 20: 380.

26. Huger WE, Jr. The anatomic rationale for abdominal lipectomy. Am Surg. 1979: 45: 612.

27. Jamieson EBA. Illustrations of Regional Anatomy. Section III, Abdomen and Section VII, Lower limb. E \& S Livingston Ltd. Edinburgh. 1936.

28. Ellis H. Clinical Anatomy. $5 \mathrm{t}^{\mathrm{h}}$ Edition. Blackwell Scientific Publications. Oxford, London, Edinburgh, Melbourne. 1966, 53-62.

29. Romanes GJ. Cunnigham's Manual of Practical Anatomy. Thorax and Abdomen. $13^{\text {th }}$ Edition. Volume 2. London Oxford University Press. 1968; 93-111.

30. Jamieson EBA. Companion to Manuals of Anatomy. $7^{\text {th }}$ Ed. London. Oxford University Press. 1965; 359371.

31. Warwick and Williams. (Eds.). Gray's Anatomy. 35th British Edition. Philadelphia. W.B. Saunders. 1973; 519-527, 656-673, 1047-1052, 1254-1257.

32. Grant JCB. Grant's Atlas of Anatomy. 6th Edition. Baltimore. Williams \& Wilkins, 1972. 105-112.

33. Clemente CD. Anatomy, A Regional Atlas of the Human Body. $4^{\text {th }}$ Edition. Williams and Wilkins. 1996; 246-270

34. Smith LF Sr., Smith LF Jr. Safely Combining Abdominoplasty with Aggressive Abdominal Liposuction Based on Perforator Vessels: Technique and a Review of 300 Consecutive Cases. Plast. Reconstr. Surg. 2015; 135: 1357.

35. Shermak MA, Rotellini-Coltvet LA, Chang D. Seroma development following body contouring surgery for massive weight loss: Patient risk factors and treatment strategies. Plast Reconstr Surg. 2008; 122:280-288.

36. Gusenoff JA. Prevention and management of complications in body contouring surgery. Clin Plast Surg. 2014; 41: 805-818. 
Chapter 7

37. Saam S, Tourani SS, Taylor I, Ashton MWM. Scarpa Fascia Preservation in Abdominoplasty: Does It Preserve the Lymphatics? Plast. Reconstr. Surg. 136: 258, 2015.

38. Janis JE, Khansa L, Khansa I. Strategies for Postoperative Seroma Prevention: A Systematic Review. Plast Reconstr Surg. 2016; 138: 240. 
Chapter 8.

Abdominoplasty with Circumferential Liposuction, DVT, Local Anesthesia: How Much Proof is Needed

Brauman, van der Hulst and van der Lei: Accepted PRS. In Press

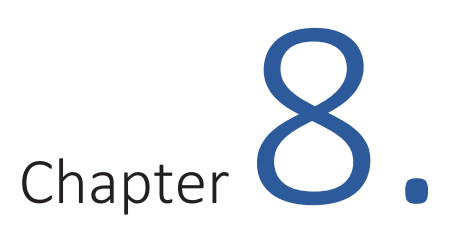

Letter to Editor

PLASTIC AND RECONSTRUCTIVE SURGERY.

Submitted 11/08/2018

Accepted 11/16/2018

IN PRESS 



\title{
Chapter 8.
}

\author{
Abdominoplasty with Circumferential Liposuction, DVT, Local \\ Anesthesia: How Much Proof is Needed? \\ Brauman, van de Hulst and van der Lei
}

Plastic and Reconstructive Surgery: Letter to editor

$11 / 08 / 2018$

Dear Sir,

\section{Abdominoplasty with Circumferential Liposuction, DVT, Local Anesthesia: How Much Proof is Needed?}

We read with interest the article authored by Sozer et. al., describing their vast experience with "abdominoplasty with concurrent circumferential liposuction." ${ }^{1}$ Their aesthetic results are exceptional and their concept of approaching the entire trunk as an aesthetic unit is indeed how the abdominoplasty patient should be approached.

We had already described the same central unit approach to abdominoplasty with circumferential liposuction, an approach we have been practicing since the 1990's and published in a series of articles titled Liposuction Abdominoplasty. ${ }^{2,3}$ Our articles described abdominoplasties with concurrent circumferential liposuction, performed under local anesthesia with sedation, an approach we continue to prefer as a means to prevent deep venous thrombosis/pulmonary embolism - DVT/PE/VTE. In a series now totaling now more than 600 consecutive patients since 1996, we have successfully prevented and avoided DVT/PE

Our approach to abdominoplasties with circumferential liposuction is identical to that of Sozer's, however our surgeries figure more complex and our patient population is at least as challenging. Our patients' mean BMI was 29.34 the supernatant fat removed - mean- $2174.7 \mathrm{ml}$, maximum - $8200 \mathrm{ml}, 59.1 \%$ underwent concurrent procedures and additional contouring, $16.3 \%$ buttock fat grafting, 52 had massive weight loss, necessitating body uplifts, and so on. Our flap undermining extends beyond the costal margin to the inframammary crease, higher and wider than that described by Sozer, and $90 \%$ of our abdominoplasties would be classified - extended abdominoplasties using Sozer's classification. Our abdominoplasty technique employs a rich network of vessels lining the flap's deep surface to distribute blood supply to the undermined flap, resulting in an increased flap excursion and obviating the need for vertical incisions. Our complications included $2 \%$ seromas, $1 \%$, infected seromas, and One hematoma.

15 of Sozer's patients sustained DVTs despite anticoagulant prophylaxis, 3 of the 15 experienced pulmonary emboli and one of the 3 died (1:1000 deaths). Thus, despite following practice recommendations, VTE still occurred. This raises questions about the validity of practice recommendations on DVT prevention. Furthermore, anticoagulants in abdominoplasty have risks. Dini et. al. sustained $29.6 \%$ serious bleeding complication and had to discontinue their study of anticoagulants in abdominoplasties. ${ }^{4}$ We sustained one hematoma - the only time we used anticoagulants. But then, we have never experienced DVT in the more than 600 abdominoplasties since 1996 and in hundreds of additional body contourings since 1990 .

Although abdominoplasties are known risk factors for DVT which is likely potentiated by general anesthesia, abdominoplasties under local anesthesia with sedation have been largely overlooked. Under general anesthesia muscle tone is reduced, lessening the effectiveness of the muscle pump mechanism, positive pressure ventilation lessens the effectiveness of the respiratory pump, further reducing venous return. ${ }^{5}$ Sedation better maintains 
muscle tone and enables evaluation of abdominal laxity under truer physiologic conditions preventing abdominal wall overtightening, respiratory dysfunction and a further increase in intra-abdominal pressure. ${ }^{6}$

2012 safety data from Florida and Alabama implicating abdominoplasties under general anesthesia in surgical deaths and the information in this letter back our call to readers to consider abdominoplasty under local anesthesia.

Sincerely Yours,

D. Brauman, MBBS, MD., R. van der Hulst, MD., Ph.D., and B. van der Lei, MD., Ph.D.

\section{References}

1. Sozer SA, Basaran K, Alim H. Abdominoplasty with circumferential liposuction: a review of 1000 consecutive cases. Plast Recoinstruct Surg 142; 891, 2018.

2. Brauman D. Liposuction abdominoplasty: An evolving concept. Plast Reconstr Surg. 112: 288, 2003.

3. Brauman D, van der Hulst RWJ, van der Lei B. Liposuction Assisted Abdominoplasty (LAA): An Enhanced Abdominoplasty Technique.

4. Dini, G.M., Corsi M.C., Gianini F.L., Ferreira, A.L.M. How Safe Is Thromboprophylaxis in Abdominoplasty? Plast. Reconstr. Surg. 2012; 130: 851e.

5. Klabunde RE. Factors promoting venous return. In, Cardiovascular Physiology Concepts, 2nd edition. 2012; Lippincott Williams \& Wilkins.

6. Blatnik JA. Predicting Severe Postoperative Respiratory Complications following Abdominal Wall Reconstruction. Plast Reconstr Surg . 2012; 130: 83. 
Chapter 9.

General Discussion and Future Perspectives

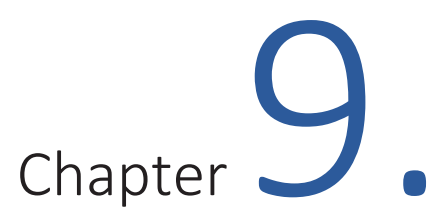

General Discussion

and

Future Perspectives 



\section{Chapter 9.}

\section{General Discussion and Future Perspectives}

\section{General Discussion and Future Perspectives}

This thesis describes the evolution of an approach to outpatient ambulatory surgery under local anesthesia from its inception in 1982 to the present. It focuses on body contouring surgery revolutionized by the introduction of liposuction to the US by Illouz in $1982 .{ }^{1}$ The thesis contains a series of published, peer reviewed articles and texts, describing and discussing techniques and concepts developed and updated over the years, supported by prospectively collected data since 1990.

Liposuction Assisted Abdominoplasty (LAA) is a comprehensive approach to body contouring, as it comprises a surgical technique, an approach to local anesthesia and sedation, and a series of concepts. LAA includes both liposuction and excisional body surgery. The liposuction technique that preceded and then evolved into the LAA technique was coined Anatomical Liposuction because it was defined as liposuction at predetermined anatomical layers or tissue planes. In the case of abdominal liposuction, liposuction was first performed at the layer that adjoined the muscular abdominal wall to separate the subcutaneous tissue from the muscular abdominal wall, to define the deep border of the subcutaneous tissue and allow an accurate estimation of the thickness of the tissue to be liposuctioned. ${ }^{2,3}$ Furthermore, deep layer liposuction created a safe zone that prevented abdominal wall perforations and allowed precise thinning of the subcutaneous tissue. The anatomical liposuction technique also targeted other layers in order to address special requirements such as, contracting the skin with Superficial Liposuction and as well as allowing the loosened fat to act as a fat graft - smoothing the skin surface.

\section{Chapter by Chapter}

The ensuing discussion summarizes the thesis chapter by chapter.

Chapter 1. This chapter recanted the history of the abdominoplasty procedure from its inception in the late 19th century as purely functional surgery into an aesthetic and functional procedure. ${ }^{4}$ It described a procedure that became popular while becoming increasingly scrutinized as problems and complications emerged.

The issue of venous thromboembolism (VTE) became associated with abdominoplasty when Grazer in 1973, and Grazer and Goldwyn in 1977 brought attention to the VTE risk of 
abdominoplasties. ${ }^{5,6}$ Since then, abdominoplasties have been recognized as independent risk factors for VTE and although general anesthesia had long been recognized an independent risk factor for VTE, the plastic surgery literature fully embraced the general anesthesia risk for DVT when Stuzin et al. and Reinisch et al. in 2001 reported on the unexpected occurrence of VTE in facelifts under general anesthesia. ${ }^{7-11}$ Nevertheless, despite direct and indirect evidence there continues to be a reluctance to implicate general anesthesia as a major cause for VTE in abdominoplasty. ${ }^{11}$

Chapter 1 also described the state of the art in 1996 when LAA was conceived. At that time, the plastic surgical literature advised against combining liposuction and abdominoplasty, warning of an increased complication rate and a risk to the flap's circulation from liposuction. ${ }^{12,13}$ However, intra-operative observations led to a different conclusion, that dissecting the flap with liposuction was superior to a dissection with existing surgical tools (such as, cutting or cautery) in protecting flap circulation.

Chapter 2. A proposition that liposuction be performed under local anesthesia and that less infiltrate be utilized was made in $2000 .{ }^{14}$ The article also suggested the use of a different hemodynamic classification based on the overall fluid infiltrated rather than the volume of fat removed, it argued that the volume of the infiltrate is a better indicator of the hemodynamic stress-load of liposuction than the volume of aspirate and it also inferred that liposuction under local was safer than under general anesthesia. ${ }^{15,16}$ The article was written in response to reports of deaths from complications of liposuction under general anesthesia. The reports described general anesthesia mishaps, deaths from VTE and deaths due to lidocaine toxicity and fluid overload. ${ }^{17,18}$ These complications likely occurred because plastic surgeons performed the then popular tumescent liposuction under general anesthesia. Not constrained by their patients' discomfort, they were able to treat larger body surface areas than their dermatologic counterparts. In the 2000 article, the link of VTE to general anesthesia had to be made indirectly, by the fact that a plastic surgeon was (uncharacteristically) performing liposuction under local anesthesia and by the reference to VTE and general anesthesia in the article's conclusion. This, because it was not until one year later in 2001 that Stuzin et al. finally demonstrated the connection between general anesthesia and VTE in aesthetic surgery. ${ }^{8}$

Chapter 3. The article formally introduced the Liposuction-Abdominoplasty approach (now LAA) with its updated anesthesia and sedation techniques. ${ }^{19,20} 60$ consecutive body contouring patients of whom 48 underwent abdominoplasties safely without DVT, flap necrosis, or other complications were presented. The results of this study were compared to other published data that associated wide undermining with flap necrosis, concluding that the absence of flap necrosis and the reduction in the incidence of seroma was due to less undermining inherent in the Liposuction Assisted Abdominoplasty (LAA) technique. ${ }^{12,19}$ The LAA flap is a mobile island flap with a set thickness and a well-defined deep layer. The flap's mobility is extended by the targeted 
disruptions and divisions of the skin retaining ligaments, it therefore has a longer downward range of excursion since it is tethered by flexible neurovascular perforators and not restrained by the stiff skin retaining ligaments. Furthermore, the anatomical liposuction of the interface between the muscular abdominal wall and the subcutaneous tissue creates a well-defined layer of a collapsed subcutaneous tissue scaffolding coined the supporting tissue layer, composed of fibrous tissue containing a rich network of blood vessels, nerves and lymphatics. The supporting tissue layer distributes blood supply originating in the proximally based neurovascular perforators, to the distal edges of the flap, enabling a longer flap excursion and ample blood (and nerve) supply that make possible in most cases an immediate post-operative upright (not flexed) posture and prevents marginal necrosis and wound complications. ${ }^{21}$ Furthermore, the supporting tissue layer, lining the deep surface of the flap provides a sharp border that permits a precise determination of the thickness and evenness of the flap.

The 2003 article also introduced an approach to diastasis recti repair and the estimation of the intra-abdominal fat content/volume. The article differentiated between abdominal wall laxity and protrusion concluding that, without sufficient laxity diastasis repair will not endure, that the mere presence of diastasis should not be considered an absolute indication for diastasis repair, and that diastasis repair was unlikely to endure in the presence of a large intra-abdominal fat volume with minimal laxity.

Chapters 4 \& 4 a. Chapter 4 was an intraoperative anatomical study of 92 consecutive abdominoplasties in which the width, length and site of the widest diastasis, was compared to the site of the maximal abdominal wall protrusion in the upright patient. ${ }^{22-24}$ The study challenged the notion that abdominal wall protrusions are caused by diastasis only and that the larger the diastasis the larger the protrusion. Instead, the findings indicated that abdominal wall protrusions are caused by the stretching of the entire abdominal wall, not only the linea alba and that significant abdominal wall protrusions occur without diastasis. Furthermore, that the site of the maximal abdominal protrusion may not correspond to the site of the widest diastasis, that flat abdomens may exhibit a diastasis, that the presence of diastasis is not synonymous with the presence of a protrusion and finally, that $82 \%$ of diastases are less than 2 inches wide and that only $2 \%$ are wider than 2.5 inches regardless of the size of the muscular-fascial protrusion.

Chapters 4, \& 4a tackled Diastasis Recti repair, an integral component of abdominoplasty surgery. Diastasis repair reduces abdominal protrusion and increases core strength by aligning the Recti muscles in the midline. ${ }^{25,26}$ Aligning the muscles against the midline greatly enhances their contraction efficiency however, the reduction in the abdominal protrusion increases intra-abdominal pressure, which likely contributes to VTE and respiratory complications. ${ }^{27-32}$

The evaluation of the muscular-fascial abdominal wall is complex. It has to include the evaluation of the intra-abdominal fat volume, the evaluation of abdominal wall laxity (overstretching), the 
evaluation of the lumbar lordotic curve, the maximal protruding segment of the muscular abdominal wall, previous midline surgeries, and the like. ${ }^{22}$

Chapter 5. Chapter 5 is a PRS article that discussed the experience with 337 patients who underwent LAA. ${ }^{21}$ There were no serious complications or DVT and the flap dissection technique using hydro and lipo-dissection was described as well as three principles that guide the sedation technique. They were, the use of principal drugs that are reversible by antagonists, monitoring of the blood oxygen level without supplemental oxygen - enabling a faster response time to a drop in pulse oximeter readings, and a slow and gradual induction of sedation. ${ }^{21,33}$ Circumferential torso liposuction was an integral component of the surgical technique in all patients, $31 \%$ of patients underwent liposuction of one more areas in addition to the torso liposuction, $13 \%$ underwent liposuction of 2 additional areas, and 23\% underwent buttock fat grafting. 15 of the 337 patients had undergone modified body lift/massive weight loss procedures, and 19 of the 337 had concomitant hernia repairs. $52 \%$ of patients had a history of prior abdominal surgeries and many others had various medical conditions in keeping with their ages that ranged from 21 to 72 years old. The patients therefore represented a normal cross section of the population and thereby demonstrated that the Liposuction Assisted Abdominoplasty (LAA) approach was applicable to all abdominoplasty candidates.

The discussion focused on describing in detail the Liposuction Assisted Abdominoplasty (LAA) technique and highlighting the fundamental differences between it and the illouz technique, popularized by Saldanha in 2001 and known as Saldanha's Lipoabdominoplasty. ${ }^{34,35}$ Importantly, the Liposuction Assisted Abdominoplasty (LAA) technique was based on first separating the muscular abdominal wall from the skin and subcutaneous layer with liposuction. The blunt-tip liposuction spared the elastic neurovascular pedicles while piercing/disrupting the stiff skin retaining ligaments and teasing off the adipose tissue from the layer closest to the deep fascia. This resulted in the separation of the panniculus from the deep fascia and the creation of a supporting tissue layer of blood vessels, nerves and lymphatics held together by a collapsed fibrous tissue scaffolding. The supporting tissue layer not only nourishes the flap but also creates a well-defined dense layer that functions as a deep border to the flap ensuring even flap thickness.

In contrast, the Illouz/Saldanha Lipoabdominoplasty technique uses liposuction on the greater part of the flap (except the most superficial $1 \mathrm{~cm}$ below the skin) without creating a well-defined and even flap and without actually separating it from the abdominal wall. Furthermore, LAA targeted the skin retaining ligaments, first disrupting (tearing) them with the closed liposuction performed under tension and then incising them under direct vision as the flap was being dissected away from the deep fascia. The blood-rich supporting tissue layer ended distributing blood supply to the distal edge of the flap where liposuction was again utilized to separate the panniculus to be discarded creating a mesentery-like extension of the supporting tissue layer, 
extending for about a quarter of an inch beyond the distal flap border. Thus, compared to Saldanha's lipoabdominoplasty where the panniculus is first excised, the LAA panniculus is excised last so that blood supply to the distal edge can be demonstrated by bleeding from the distal edge, usually requiring light cautery. The technique was demonstrated (with videotape) in a 2018 PRS Global Open article - Chapter 7 -that described the experience with 593 consecutive cases. $^{36}$ Finally, the profuse blood supply permitted (with increased experience) additional discontinuous undermining and division of some neurovascular perforators under vision, to further extend the flap's reach, since the supporting tissue layer was relied on (as a vascular lining of the entire undersurface of the flap) to distribute the blood supply originating in the proximal perforators. Furthermore, Scarpa's fascia (when undamaged by previous $C$ sections etc.) was preserved and vessels originating from the femoral artery were able to "supercharge" the flap with added blood supply to augment superior epigastric and spinal perforators.

The article ended with a brief discussion and a definition of seromas as fluid collections that persist beyond 6 weeks. Treatment of seromas with steroids was described, and segmental diastasis repairs, that only repair the most protruding segment of the diastasis to reduce tension and avoid a rise in intraabdominal pressure, were also noted.

Chapter 6. The chapter formatted in an article format and was devoted to the performance of body contouring under local anesthesia with sedation as a means to prevent DVT and PE. ${ }^{37}$ It concluded that although literature searches of DVT/PE had been conducted by authors researching this topic, the search is hampered by different and overlapping databases (and articles), as well as the focus by different authors on distinct aspects of this topic such as the etiology, prevention, chemoprophylaxis, magnitude of surgeries and so on. Most distracting is the grouping of DVT and PE under the heading of VTE, not allowing a precise and separate data analysis for DVT's and PE's.

The DVT prevention protocols utilized by the author have been common sense measures, most of which are well known. But the article emphasizes that the full prevention effect of these measures can only be achieved when practiced in their entirety and adhered to. Chemoprophylaxis is problematic in excisional body contouring and therefore practice guidelines are difficult to draft, also, different protocols and anticoagulants are utilized and therefore conclusions are difficult to come by. Furthermore, despite chemoprophylaxis, DVT continues to plague abdominoplasties under general anesthesia and Chapter 8 of this thesis, has given voice to this problem. ${ }^{40}$

In spite of the above restrictions in data analysis, the only plausible conclusion that can be reached so far is that abdominoplasty under local anesthesia with sedation offers the best DVT prophylaxis, since no reports of DVT have been thus far reported. That is not to say that this approach is immune to this problem and that DVT has not occurred, however it definitely appears that local anesthesia with sedation is an effective DVT prevention protocol. 
Chapter 7. Chapter 7 is a PRS Global open article summarizing the experience with 593 consecutive LAA cases under local anesthesia with sedation, underscoring the safety of this approach. ${ }^{36}$ Current experience with this approach now numbers 600 consecutive patients studied prospectively and longitudinally. The experience with this technique and especially with the last group of 256 patients from 2009-2018 demonstrated its safety and efficacy. The article also emphasizes the flexibility of $L A A$ technique to be used under general anesthesia with equal safety.

The article discusses the issue of seroma in some length. LAA attempts to prevent seroma formation by preservation of perforators attaching the panniculus to the abdominal wall, reducing dead space and by the preservation of lymphatics with liposuction. Furthermore, LAA preserves Scarpa's fascia and liposuctions tissues superficial and deep to it, and although a recent article demonstrated that the lymphatic drainage from the lower abdomen courses superficial to Scarpa and pierces the fascia low, 2-3 cm superior to the inguinal ligament, liposuction of the tissue superficial and deep to Scarpa most likely preserves many of the lymphatics now coursing on Scarpa's superficial surface within the preserved layer of supporting tissue. ${ }^{41}$ Nonetheless, lower abdominal surgery, such as Caesarian Sections, prevalent in the US, would render preservation of the fascia moot, because the surgery disrupts the lymphatics and may predisposes to seroma formation.

Finally, the treatment of seromas by the author with intralesional and systemic steroids has been effective for the past 20 years and in keeping with seromas' inflammatory nature. More recently sclerotherapy by a radiologist interested in the treatment of fluid collections has been helpful and patients are referred to him for treatment. ${ }^{21}$

Chapter 8. Chapter 8 is a letter to the editor commenting on an article by Sozer et al, who reported on 1000 consecutive cases of abdominoplasty, naming their approach of abdominoplasty with circumferential torso liposuction, a central unit approach. ${ }^{40}$ Sozer et al. incurred 15 cases of DVT and 1 death from PE despite routine prophylaxis with enoxaparin and venous sonograms. ${ }^{42}$ The letter points out that the central unit approach had been previously carried out and published in $600+$ cases under local anesthesia since 1996 without deaths or DVT and the letter asked how much proof is needed to satisfy the requirement for evidence that local anesthesia with sedation is an effective DVT prophylaxis? ${ }^{19,36,43,44}$

\section{Future Perspectives}

DVT and PE prevention have been a central topic in this thesis. DVT/PE had been first brought to the attention of the plastic surgery profession by Grazer in 1973 and again by Grazer and Goldwyn in 1977 and has vexed body contouring surgery ever since, despite practice guidelines, 
chemoprophylaxis and prevention protocols. ${ }^{42}$ This thesis discussed DVT and PE in some detail and through several publications (included in this thesis), recommended that local anesthesia with sedation be accepted an effective method of DVT prevention, suggesting that there is ample evidence in the literature to support this opinion. A suggestion was made to replace the term VTE with the terms PE and DVT, and when reporting DVT/PE events to specify if the number of $\mathrm{PE}$ cases is included in the number of DVTs reported. This, to allow accurate statistical analysis. This thesis also submits that DVT prevention protocols are effective only if they are followed in their entirety. It is hoped that these suggestions will be publicized and accepted.

LAA is a technique that uses liposuction as a dissection tool. The LAA title was coined to differentiate this technique from other lipo-abdominoplasty techniques. LAA is also different from approaches that describe liposuction with or as an adjunct to abdominoplasties since, liposuction + abdominoplasty approaches appear to retain the belief that liposuction is more injurious to the flap and therefore either the abdominoplasty or liposuction (or both) are constrained. It is hoped that the name change will help popularize this technique since only wide usage can verify the benefits of this technique.

Current practice is to classify liposuction by the volume of aspirate and volumes higher than $5000 \mathrm{cc}$ are considered high. Although this standard has worked reasonably well in reducing excessive liposuction, it is too restrictive for some large patients with localized adiposities and too permissive for small patients who undergo liposuction of multiple areas. Because tumescent liposuction for example, may infiltrate roughly twice the volume of blood and extract much less than $5000 \mathrm{cc}$ in an individual whose cardiac reserve may not be sufficient to tolerate the infiltration of so much fluid in a very short period of time. It is hoped that this suggestion will be investigated.

The current standard for diastasis repair is a xiphoid to pubis repair. Nevertheless, repair of the most protruding segment of the diastasis - a tapered segmental repair, ought to be studied as a means to prevent respiratory complications and DVT, potentiated by the increase in intraabdominal pressure. Furthermore, segmental repairs prevent postoperative epigastric protrusions in selected individuals with large intraabdominal volumes, and likely increase the durability of diastasis repairs. Ultimately, an algorithm to guide the decision to perform diastasis repairs ought to be developed.

Massive weight loss patients have been found to incur a higher incidence of seroma formation. ${ }^{36}$ Intra-operative clinical observations suggest that weight loss results in the loss of fat, but that the supporting tissue scaffolding containing dilated blood vessels, lymphatics and nerves persists. This can explain the greater blood loss and higher incidence of seromas in these patients. This intraoperative observation needs to be substantiated by others. 
Body uplifts and circumferential abdominoplasties are categorized by the extent of their surgery. It is suggested that they should be additionally classified as massive weight loss surgeries, a term used in this thesis. ${ }^{19,21,22,36}$ The term massive weight loss does not depend on whether or not a patient had undergone bariatric surgery, because many are able to lose a great deal of weight, maintain it (if only for several years), and seek body contouring surgery. Furthermore, regardless of the improvement in peoples' obesity related medical conditions, it is not known what the sequelae of long-term morbid obesity are following weight loss surgery, and how it affects these patients' surgical stress tolerance. In any case, the metabolic syndrome (that may have preexisted) is a serious condition that should be taken into account, and the predisposition of massive weight loss patients to seromas, may indicate that their pre-existing morbid obesity left residual damage. ${ }^{45-47}$

Finally, the author had previously treated seromas effectively with systemic and intralesional steroids. ${ }^{21,36}$ A study to determine the effectiveness of this treatment undertaken at that time, was interrupted when a radiologist interested in seromas undertook their treatments and has been performing sclerotherapy at an affiliated hospital's outpatient department ever since. In light of a recent article confirming the inflammatory nature of seromas, it would be valuable to study the effectiveness of steroid treatment in cases where the integrity of a diastasis repair or the tensile strength of a healing incision is not in question. ${ }^{48}$

\section{References:}

1. Illouz YG. First international presentation on BLUNT lipoplasty technique. Presented at Shirakabe Clinic, Osaka, Japan, 1980.

2. Brauman D. Anatomical Liposuction \& Liposuction Abdominoplasty. The second Huffstadt International Conference in Groningen, The Netherlands. University Medical Centre of Groningen. November 12-13, 2010.

3. Brauman D. Anatomical Liposuction \& Liposuction Abdominoplasty: Live Surgery. Sint Lucas Andreas Hospital, Amsterdam, The Netherlands. NVEPC 6th International Live Surgery and Congress on Aesthetic Plastic Surgery Body contouring, Amsterdam, The Netherlands. December 7 \& 8, 2012.

4. Brauman D, Chapter 1. Introduction and outline of this thesis.

5. Grazer FM. Abdominoplasty. Plast. Reconstr. Surg. 1973; 51:617-623.102.

6. Grazer FM, Goldwyn RM. Abdominoplasty assessed by survey with emphasis on complications. Plast. Reconstr. Surg. 1977; 59:513-517.

7. Reinisch, J. F., Bresnick, S. D., Walker, J. W. T., and Rosso, R. F. Deep venous thrombosis and pulmonary embolus after face lift: A study of incidence and prophylaxis. Plast. Reconstr. Surg. 107: 1570, 2001.

8. Stuzin, J. M., Baker, T. J., and Baker, T. M. Deep venous thrombosis and pulmonary embolus after face lift: A study of incidence and prophylaxis (Discussion). Plast. Reconstr. Surg. 107: 1576, 2001. 
9. Daane, S. P., and Rockwell, W. B. Analysis of methods for reporting severe and mortal lipoplasty complications. Aesthetic Plast. Surg. 23: 303, 1999.

10. Venturi, M.L., Davison, S.P., Caprini, J.A. Prevention of Venous Thromboembolism in the Plastic Surgery Patient: Current Guidelines and Recommendations Aesthet Surg J (2009) 29 (5): 421-428.

11. Keyes, G.R. Commentary on: Venous Thromboembolism in the Cosmetic Patient: Analysis of 129,007. Aesthetic Surgery Journal. 2017, Vol 37(3) 350-352.

12. Dillerud, E. Abdominoplasty combined with suction lipoplasty: A study of complications, revisions, and risk factors in 487 cases. Ann. Plast. Surg. 1990; 25: 333.

13. Heppe, H. P. Combined liposuction with abdominoplasty (Letter). Plast. Reconstr. Surg. 2001; 108: 577.

14. Brauman D. Lipoplasty: A Case for a Low-volume Procedure. Aesthet Surg J 2000; 20 (5): 373-379.

15. Hemodynamic Society. What is Hemodynamics? Hemodynamicsociety. org/ hemodyn. html

16. Jones EC, Devereux RB, O'Grady MJ, Schwartz JE, Liu JE, Pickering TG, Roman MJ. Relation of Hemodynamic Volume Load to Arterial and Cardiac Size. Journal of Cardiology. May 1997; JACC Vol. 29, No. 6:1303-10.

17. Gilliland MD, Coates N. Tumescent Liposuction Complicated by Pulmonary Edema. Plastic \& Reconstructive Surgery. January 1997; 99: 1: 215-219.

18. Samdal, Frode; Amland, Petter F.; Bugge, Jan F. Plasma Lidocaine Levels During Suction-Assisted Lipectomy Using Large Doses of Dilute Lidocaine with Epinephrine. Plast. \& Reconst. Surg. 1994; 93(6):1217-1223.

19. Brauman D. Liposuction abdominoplasty: An evolving concept. Plast. Reconstr. Surg. 2003; 112:2882.

20. Brauman D. Liposuction abdominoplasty: An evolving concept. Presented at: 71st Annual Meeting of the American Society of Plastic Surgeons; November 6, 2002; San Antonio, Texas.

21. Brauman D, Capocci J. Liposuction Abdominoplasty: An advanced Body Contouring Technique. Plast. Reconstr. Surg. 2009; 124(5):1685-1695.

22. Brauman, D. Diastasis Recti: Clinical Anatomy. Plast. Reconstr. Surg. 122: 1564, 2008.

23. Brauman, D. Reply, letter to the editor. Diastasis Recti: Clinical Anatomy. Remus Repta, R., Hunstad, JP. Plast. Reconstr. Surg. 123: 1885-1886, 2009.

24. Brauman, D. Reply, letter to the editor. Factors that May Influence Failure of the Correction of the Musculoaponeurotic Deformities of the Abdomen. Nahas, FX, Barbosa M V, Ferreira LM. Plast. Reconstr. Surg. 124: 334-335, 2009.

25. Kroll PG, Arnofsky S, Leeds S, Peckham, Douglas, Rabinowitz A. Journal of Back and Musculoskeletal Rehabilitation. 2000; vol. 14, no. 1-2.

26. 24. Król, A Polak, Maciej, Szczygieł, Elżbietac, Wójcik, Paweł, Gleb. Journal of Back and Musculoskeletal Rehabilitation, 3-27-2017, pp. 1-7.

27. Saggi BH Sugerman HJ Ivatury RR Bloomfield GL. Abdominal compartment syndrome. J Trauma. 1998; 453:597-609. 
28. Watson RA Howdieshell TR. Abdominal compartment syndrome. South Med J. 1998; 914:326-332.

29. Talisman R Kaplan B Haik J et al. Measuring alterations in intra-abdominal pressure during abdominoplasty as a predictive value for possible postoperative complications. Aesthet Plast Surg. 2002; 263:189-192.

30. Tercan M Bekerecioglu M Dikensoy O et al. Effects of abdominoplasty on respiratory functions: a prospective study. Ann Plast Surg. 2002; 496:617-620.

31. Rodrigues MA Nahas FX Gomes HC Ferreira LM. Ventilatory function and intra-abdominal pressure in patients who underwent abdominoplasty with plication of the external oblique aponeurosis. Aesthet Plast Surg. 2013; 375:993-999.

32. Rodrigues MA, Nahas FX, Reis RP, et al. Does Diastasis Width Influence the variation of the IntraAbdominal Pressure After Correction of Rectus Diastasis. Aest. Plast. Surg. 2015; 35(5) 583-588.

33. Keidan I, Gravenstein D, Berkenstadt H, Ziv A, Shavit I, Sidi A. Supplemental Oxygen Compromises the Use of Pulse Oximetry for Detection of Apnea and Hypoventilation During Sedation in Simulated Pediatric Patients. Pediatrics, August 2008, 122: 2.

34. Illouz YG. A new safe and aesthetic approach to suction abdominoplasty. Aesthetic Plast Surg. 1992; $16: 237-245$.

35. Saldanha OR, de Souza Pinto EB, Matos WN, Lucon RL, Magalhães, F, and Bello ÉML. Lipoabdominoplasty without undermining. Aesthetic Surg. J. 2001; 21: 518.

36. Brauman D, van der Hulst RRWJ, van der Lei B. Liposuction Assisted Abdominoplasty (LAA): An Enhanced Abdominoplasty Technique. PRS Global Open. Plastic and Reconstructive Surgery Global Open: September 2018; Volume 6; Issue 9 p e1940.

37. Chapter 6. Brauman D, van der Hulst R, van der Lei. A Case for Local Anesthesia and Prevention Protocols for Curbing DVT in Abdominoplasty.

38. Dini, GM, Corsi MC, Gianini FL, Ferreira ALM. How Safe Is Thromboprophylaxis in Abdominoplasty? Plast. Reconstr. Surg. 2012; 130: 851e.

39. Janis JE, Khansa L, Khansa I. Strategies for Postoperative Seroma Prevention: A Systematic Review. Plast Reconstr Surg. 2016; 138: 240.

40. Chapter 8. Brauman, van der Hulst and van der Lei. Abdominoplasty with Circumferential Liposuction, DVT, Local Anesthesia: How Much Proof is Needed. Accepted PRS. In Press.

41. Saam S. Tourani, S, S, Taylor,I,. Ashton, M,W M., Scarpa Fascia Preservation in Abdominoplasty: Does It Preserve the Lymphatics? Plast. Reconstr. Surg. 2015; 136: 258.

42. Sozer SA, Basaran K, Alim H. Abdominoplasty with circumferential liposuction: a review of 1000 consecutive cases. Plast Recoinstruct Surg 142; 891, 2018.

43. Klabunde RE. Factors promoting venous return. In, Cardiovascular Physiology Concepts, 2nd edition. 2012; Lippincott Williams \& Wilkins.

44. Blatnik JA. Predicting Severe Postoperative Respiratory Complications following Abdominal Wall Reconstruction. Plast Reconstr Surg . 2012; 130: 83. 
45. Kershaw EE, and Flier JS. Adipose tissue as an endocrine organ. metabolic syndrome Hip to waist ratio J. Clin. Endocrinol. Metab. 2004; 89: 2548.

46. Yusuf S, Hawken S, Ounpuu S, et al. Obesity and the risk of myocardial infarction in 27,000 participants from 52 countries: A case-control study. Lancet. 20015; 366: 1640.

47. Romero-Corral A., Montori VM, Somers VK, et al. Association of bodyweight with total mortality and with cardiovascular events in coronary artery disease: A systematic review of cohort studies. Lancet 2006; 368: 666 .

48. Janis JE, Khansa L, Khansa I. Strategies for Postoperative Seroma Prevention: A Systematic Review. Plast Reconstr Surg. 2016; 138: 240. 

Chapter 10.

Valorization Addendum. Acknowledgement. About the Author

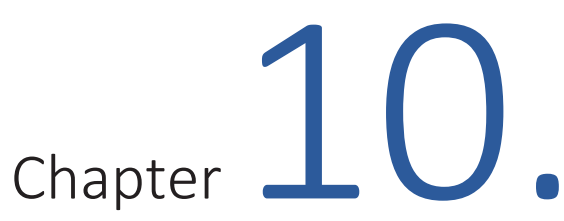

Valorization Addendum Acknowledgement About the Author 



\section{Valorization Addendum}

According to the American Society of Plastic Surgeons (ASPS), 1,790,832 aesthetic surgical procedures were performed in the US during 2017. Of these, there were 129,753 abdominoplasties, 246,354 liposuctions, and 9,075 body uplifts, Altogether 385,182 body contouring surgeries in one year. Of note, abdominoplasties more than doubled in number since 2000, when 62,713 abdominoplasties were performed. ${ }^{1}$

When assigning a value to a surgical procedure such as an abdominoplasty, it would be easy to monetize it by calculating its financial value and its contribution to the economy. Nevertheless, this would omit the considerable positive effect of such an aesthetic-reconstructive procedure on those who underwent it, despite the risks and costs. The removal of a hanging panniculus for example, improves a person's self-esteem, level of activity and day to day comfort because the discomfort, intertrigo, and backaches -helped by a tighter core are eased. Moreover, in a marketbased economy it is reasonable to assume that a person who is content and has a better selfesteem would contribute by producing and consuming more. This thesis chronicled the advances made in body contouring surgery since Grazer 1973 and Grazer and Goldwyn 1977 first pointed out the safety issues in abdominoplasty and since Illouz introduced liposuction to the US in 1981..$^{2-4}$ Since then, the general advances in anesthesia, techniques and medical care have rendered body contouring surgery safer and its outcomes more predictable. The thesis relates the author's approach to this field and the effort to further improve the safety and effectiveness of body contouring into the future.

Obesity has reached an epidemic proportion in the western world and during a visit to Amsterdam in 2012 at the NVEPC 6th International Congress on Body Contouring, a Netherlands plastic surgeon surprised me by conveying that morbid obesity was no longer an "American problem", it has also become a problem in the Netherlands. What's more, the patient I was assigned to, turned out to be a massive weight loss patient who successfully lost weight with her Lap-Band port still in place. Obesity is a public health problem. The Center for Disease Control and Prevention (CDC) website discloses that 39\% of Americans in 2015-2016 were obese, that the estimated annual medical cost of obesity in the United States was $\$ 147$ billion (in 2008), and the medical cost for people who are obese was $\$ 1,429$ higher than for normal weight adults. Morbid obesity is about $8 \%$ and the CDC considers obesity an epidemic. ${ }^{5}$

Bariatric surgery has been recognized by the National Institute of Health (NIH) to be the only effective treatment for severe obesity. Bariatric surgeries are effective in that the majority of individuals are successful in maintaining $50 \%$ or more of their excess weight loss following bariatric surgery. ${ }^{6}$ However, despite the improvement in their health, sagging tissues plague those who undergo bariatric surgery and their body image issues and discomfort lead them to seek body uplift type surgeries. According to the ASPS there was a $4000 \%$ increase in body uplifts 
since $2000 .{ }^{1}$ A staged approach to these surgeries as a means to limit complications and untoward sequelae has been described in this thesis through classifying this surgery massive weight loss surgery. ${ }^{7}$ Staging the procedures kept complications at bay and helped the understanding of the massive weight loss problem. The study of massive weight loss surgery also raised the question if pre-existing morbid obesity in it of itself, places candidates for surgery in a medically higher risk category regardless of the type of surgery planned.

According to data published on the CDC website (page last reviewed on February 5, 2018), deep vein thrombosis and pulmonary embolism (DVT/PE) is estimated to affect approximately 900,000 people in the (US, $0.1 \%-0.2 \%$ of the population) each year, although the precise number is unknown. ${ }^{8,9}$ Estimates further suggest that $60,000-100,000$ Americans die premature death due to DVT/PE each year and that 10 to $30 \%$ of those diagnosed, will die within one month of diagnosis with sudden death. Sudden death is the first symptom in about $25 \%$ of people who have a PE. It is also estimated that $50 \%$ of those who have had DVT will have long-term complications as in post-phlebitic leg syndrome, that $33 \%$ will have another DVT within 10 years, and that approximately 5 to $8 \%$ of the U.S. population has a genetic risk factor for DVT originating from disorders known as inherited thrombophilias. ${ }^{8,9}$

The health care costs of DVT/PE are estimated at $\$ 10$ billion or more each year in the United States. Over half of blood clots are related to a recent hospitalization or surgery and most of these do not occur until after discharge. ${ }^{10-13}$ Furthermore, despite estimates that as many as $70 \%$ of cases of hospital derived DVT/PE could be prevented, less than $50 \%$ of these patients receive adequate prevention measures. ${ }^{14-16}$

The statistics, even if half true are sobering. The CDC therefore recommends that best-practices, proven prevention tools and evidence-based education tools be "shared for partners and stakeholders". Accordingly, it is wished that this thesis will contribute in some measure to this effort.

\section{References}

1. American Society of Plastic Surgeons. Statistics. 2017.

2. Grazer FM. Abdominoplasty. Plast. Reconstr. Surg. 1973; 51:617-623.102.

3. Grazer FM, Goldwyn RM. Abdominoplasty assessed by survey with emphasis on complications. Plast. Reconstr. Surg. 1977; 59:513-517.

4. Illouz YG. First international presentation on BLUNT lipoplasty technique. Presented at Shirakabe Clinic, Osaka, Japan, 1980.

5. Centers for Disease Control and Prevention (CDC). Website. 2019.

6. National Institute of Health (NIH). Website. 2019. 
7. Brauman D, van der Hulst R, van der Lei. Liposuction Assisted abdominoplasty (LAA): An Enhanced Abdominoplasty Technique.

8. Beckman MG, Hooper WC, Critchley SE, Ortel TL. Venous thromboembolism: a public health concern. Am J Prev Med. 2010 ;38 (4 Suppl): S495-501. doi: 10.1016.

9. Division of Blood Disorders, National Center on Birth Defects and Developmental Disabilities, Centers for Disease Control and Prevention. Page last reviewed: February 5, 2018.

10. Streiff MB, Brady JP, Grant AM, Grosse SD, Wong B, Popovic T. Centers for Disease Control and Prevention (CDC). CDC Grand Rounds: preventing hospital-associated venous thromboembolism. MMWR Morb Mortal Wkly Rep. 2014; 63(9):190-3.

11. Heit JA, Silverstein MD, Mohr DN, Petterson TM, Lohse CM, O'Fallon WM, Melton LJ 3rd. The epidemiology of venous thromboembolism in the community. Thromb Haemost. 2001; 86:452-63.

12. Spencer FA, Emery C, Joffe SW, Pacifico L, Lessard D, Reed G, Gore JM, Goldberg RJ. Incidence rates, clinical profile, and outcomes of patients with venous thromboembolism. The Worcester VTE study. J Thromb Thrombolysis. 2009; 28:401-9.

13. Spencer F, Lessard D, Emery C, Reed G, Goldberg R. Venous thromboembolism in the outpatient setting. Arch Intern Med. 2007; 167(14):1471-5.

14. Zeidan AM, Streiff MB, Lau BD, Ahmed SR, Kraus PS, Hobson DB, Carolan H, Lambrianidi C, Horn PB, Shermock KM, Tinoco G, Siddiqui S, Haut ER. Impact of a venous thromboembolism prophylaxis "smart order set": Improved compliance, fewer events. Am J Hematol. 2013; 88:545-9.

15. Mitchell JD, Collen JF, Petteys S, Holley AB. A simple reminder system improves venous thromboembolism prophylaxis rates and reduces thrombotic events for hospitalized patients. J Thromb Haemost. 2012; 10:236-43.

16. Lau BD, Haut ER. Practices to prevent venous thromboembolism: a brief review. BMJ Qual Saf. 2014; 23:187-95.

17. Kahn S, Morrison D, Cohen J, Emed J, Tagalakis V, Roussin A, Geerts W. Interventions for implementation of thromboprophylaxis in hospitalized medical and surgical patients at risk for venous thromboembolism. Cochrane Database Syst Rev. 2013; 7:CD008201-CD. 



\section{Acknowledgements}

I would like to acknowledge and extend special thanks and a deep gratitude to Prof. Dr. Berend van der Lei, my good friend and trusted colleague. Dr. van der Lei and I teamed up on the approach to body contouring he named Liposuction Assisted Abdominoplasty (LAA) and we continue to share ideas and concepts with each other. He has a personal approach to LAA, and we were able to share both our approaches at the yearly meeting of the Aesthetic Surgery Society at the Javitz convention center in New York City in 2013. Dr. van der Lei put the LAA approach on the map when he graciously invited me to present at the University of Groningen and later to present and demonstrate the techniques at the International Aesthetic Surgery Conference in Amsterdam hosted by the Netherlands Society of Plastic Surgery. I am deeply grateful.

I would like to extend special thanks to Prof. Dr. Rene van der Hulst who became my promoter and lent his good name to this work. It takes a great deal of professional competence, confidence in one's knowledge, and generosity, to recognize and support a colleague's work. Both doctors van der Lei and van der Hulst share this selfless and generous attribute, an attribute so important in a mentor, teacher and a department head. Dr. van der Hulst has also generously contributed valuable opinions and objectively critiqued this work. I am thankful.

I am deeply grateful to the love of my life and wife of 42 years, Sheryl, who deserves much better after all these years than to bear my being glued to a computer screen.

Lastly, I would like to extend my appreciation and friendship to the surgeons and scientists I was privileged to get to know in The Netherlands. I was welcomed and felt a sense of belonging and warmth one rarely experiences in meetings with colleagues. I look forward to a continued collaboration with my colleagues, in the spirit of international cooperation. 



\section{About the Author/Resume}

Daniel Brauman, MBBS, MD, FACS

\section{Curriculum Vitae:}

Birth Date: $\quad$ July 9, 1946

Army Service: $\quad 1^{\text {st }}$ Lieutenant -Army- IDF

1964-1967

\section{Education:}

\section{MB. BS. (Hon)}

Faculty of Medicine, The University of Sydney,

1967-1973

Sydney, Australia.

Awarded, Commonwealth Scholarship.

1969-1974

General Surgery

1975-1979

Albert Einstein College of Medicine N.Y.

Board Certified - General Surgery

1980, (Certif.\#25964)

Plastic \& Reconstructive Surgery

$1979-1981$

Montefiore-Albert Einstein College of Medicine N.Y.

Board Certified - Plastic \& Reconstructive Surgery

1983, (Certif. \#2793)

Microsurgery \& Hand Surgery-Fellowship

1981-1982

Rush Presbyterian, St Lukes Medical Center,

Chicago, IL.

Fellow American College of Surgeons (FACS).

1985

MD. The University of the State of New York.

1990

\section{Appointments:}


Reviewer - Mayo Clinic - Journal of Clinical Anatomy

Reviewer - Plastic and Reconstructive Surgery journal

Reviewer - Aesthetic Surgery journal

Past- Member-Board of Directors - American Cancer Society - Westchester

Past- Clinical Instructor - General Surgery - NY Medical College

Past- Clinical Instructor - Plastic Surgery - Albert Einstein College of medicine

Assistant Professor-Plastic Surgery - Weill Medical College of Cornell University

\section{Professional Organizations:}

American College of Surgeons

American College of Surgeons - New York Chapter

American Society of Plastic Surgeons

American Society for Aesthetic Plastic Surgery

International Society for Aesthetic Plastic Surgeons

Board of Directors, American Cancer Society-past

SUGUNA, Sydney University Graduates Union North America

Surgical Society of New York Medical College

New York State Regional Society of Plastic Surgeons

Westchester County Medical Society

New York State Medical Society

\section{Staff Appointments:}

Attending Surgeon - Westchester County Medical Center 1981-current

Attending Surgeon - White Plains Hospital Medical Center 1985- current

\section{Publications:}

1. Brauman D. Implantable prosthetic devices. European Patent Certificate of Grant \# 0174141 August 22, 1985.

2. Brauman D. Breast prosthesis. In: Love E., Lennox-Kerr P. Editors. Elsevier International Medical Textiles. 1986;2:11:3-4.

3. Brauman D. Implantable prosthetic devices. United States Patent \# 4,648,880 March 10, 1987.

4. Strauch B., Sharzer L., Brauman D. Chapter 14: Innervated free flaps for Sensibility and Coverage In: Urbaniak R., editor. Microsurgery for Major Limb Reconstruction. CV Mosby Company. 1987:112-119. 
5. Brauman D. Implantable prosthetic devices. United States Patent \# 4,820,303 April 11, 1989.

6. Brauman D. Implantable prosthetic devices. United States Patent \# 4,963,150 October 16, 1990.

7. Brauman D. Implantable prosthetic devices. United States Patent: Reexamination Certificate \# B1 4,963,150 October 4, 1994.

8. Brauman D. Reduction Mammaplasty by Suction Alone. Letter to the Editor. Plast. Reconstr. Surg. 1994; 94:1095.

9. Brauman D. Implantable prosthetic devices. United States Patent: Reissue \# RE 35,391 December 3, 1996.

10. Brauman D. Lipoplasty: A Case for a Low -volume Procedure. Aesthetic Plastic Surgery. $2000 ; 20: 5: 373-380$.

11. Liposuction Abdominoplasty: An evolving concept. Presented at the ASPS / PSEF / ASMS $71^{\text {st }}$ annual scientific meeting November 2-6, 2002. San Antonio, Texas.

12. Brauman D. Liposuction Abdominoplasty: An evolving concept. Plast. Reconstr. Surg. July 2003;112;1:288-298.

13. Liposuction Breast Reduction. Letter to the Editor. Plast. Reconstr. Surg. October 2005, $116 ; 5 ; 1558-1559$.

14. Brauman D. Diastasis Recti: Clinical Anatomy. Plast. Reconstr. Surg. November 2008, 112;5; 1564-1569

15. Brauman D. Reply, Letter to the Editor. Diastasis Recti: Clinical Anatomy. Plast. Reconstr. Surg. 123(6):1885-1886, June 2009.

16. Brauman D. Reply, Letter to the Editor. Factors That May Affect. Plast. Reconstr. Surg. 124(1):334-335, July 2009.

17. Brauman D, Capocci J. Liposuction Abdominoplasty: An advanced Body Contouring Technique. Plast. Reconstr. Surg. 124(5):1685-1695, November 2009.

18. Brauman D. Facelifts, Eyelids Do's and Don'ts. Sydney University Graduates Union of North America. October 11-13, 2013. Charlotte, North Carolina. SUGUNA, October 13, p14, 2013.

19. Brauman D. Textured Breast Implants and Spherical Capsular Contracture. Aesth. Surg. J. 6/20/2018; sjy136.

20. Brauman D, van der Hulst RRWJ, van der Lei B. Liposuction Assisted Abdominoplasty (LAA): An Enhanced Abdominoplasty Technique. PRS Global Open. Plastic and Reconstructive Surgery Global Open: September 2018; Volume 6; Issue 9 p e1940.

21. Brauman, van der Hulst and van der Lei. Abdominoplasty with Circumferential Liposuction, DVT, Local Anesthesia: How Much Proof is Needed. Accepted PRS. In Print.

22. PRS-D-18-02799, Brauman. Rough Textured Silicone Implants, Bacterial Biofilms, and Capsular Contracture. Plast Reconstr Surg. Accepted for publication. In Print. 
Chapter 10

$-152$. 